\title{
Relaxed extragradient methods for systems of variational inequalities
}

\section{Lu-Chuan Ceng ${ }^{1,2}$ and Ching-Feng Wen ${ }^{3,4 *}$}

${ }^{*}$ Correspondence: cfwen@kmu.edu.tw

${ }^{3}$ Center for Fundamental Science, Kaohsiung Medical University,

Kaohsiung, 807, Taiwan

${ }^{4}$ Center of Nonlinear Analysis and Optimization, Kaohsiung Medical University, Kaohsiung, 807, Taiwan Full list of author information is available at the end of the article

\begin{abstract}
In this paper, we introduce a relaxed extragradient iterative algorithm for finding a common element of the set of solutions of a general mixed equilibrium problem, the set of solutions of general system of variational inequalities, the set of solutions of finitely many variational inclusions, and the set of common fixed points of finitely many nonexpansive mappings and a strict pseudocontraction in a real Hilbert space. The iterative algorithm is based on Korpelevich's extragradient method, the viscosity approximation method, Mann's iterative method, and the strongly positive bounded linear operator approach. We derive the strong convergence of the iterative algorithm to a common element of these sets, which also solves some hierarchical minimization.
\end{abstract}

MSC: 49J30; 47H09; 47J20; 49M05

Keywords: hybrid viscosity iterative algorithm; general mixed equilibrium; general system of variational inequalities; nonexpansive mapping; inverse-strongly monotone mapping

\section{Introduction}

Let $H$ be a real Hilbert space with inner product $\langle\cdot, \cdot\rangle$ and norm $\|\cdot\|, C$ be a nonempty closed convex subset of $H$, and $P_{C}$ be the metric projection of $H$ onto $C$. Let $S: C \rightarrow C$ be a self-mapping on $C$. We denote by $\operatorname{Fix}(S)$ the set of fixed points of $S$ and by $\mathbf{R}$ the set of all real numbers. A mapping $V$ is called strongly positive on $H$ if there exists a constant $\bar{\gamma}>0$ such that

$$
\langle V x, x\rangle \geq \bar{\gamma}\|x\|^{2}, \quad \forall x \in H .
$$

A mapping $A: C \rightarrow H$ is called $L$-Lipschitz-continuous if there exists a constant $L \geq 0$ such that

$$
\|A x-A y\| \leq L\|x-y\|, \quad \forall x, y \in C .
$$

In particular, if $L=1$ then $A$ is called a nonexpansive mapping; if $L \in[0,1)$ then $A$ is called a contraction. A mapping $T: C \rightarrow C$ is called $\xi$-strictly pseudocontractive if there exists a constant $\xi \in[0,1)$ such that

$$
\|T x-T y\|^{2} \leq\|x-y\|^{2}+\xi\|(I-T) x-(I-T) y\|^{2}, \quad \forall x, y \in C .
$$

In particular, if $\xi=0$, then $T$ is a nonexpansive mapping.

(c) 2015 Ceng and Wen; licensee Springer. This article is distributed under the terms of the Creative Commons Attribution 4.0 International License (http://creativecommons.org/licenses/by/4.0/), which permits unrestricted use, distribution, and reproduction in any medium, provided you give appropriate credit to the original author(s) and the source, provide a link to the Creative Commons license, and indicate if changes were made. 
Let $A: C \rightarrow H$ be a nonlinear mapping on $C$. We consider the following variational inequality problem (VIP): find a point $\bar{x} \in C$ such that

$$
\langle A \bar{x}, y-\bar{x}\rangle \geq 0, \quad \forall y \in C .
$$

The solution set of VIP (1.1) is denoted by $\operatorname{VI}(C, A)$.

The VIP (1.1) was first discussed by Lions [1]. There are many applications of VIP (1.1) in various fields; see e.g., $[2,3]$. It is well known that, if $A$ is a strongly monotone and Lipschitz-continuous mapping on $C$, then VIP (1.1) has a unique solution. In 1976, Korpelevich [4] proposed an iterative algorithm for solving VIP (1.1) in Euclidean space $\mathbf{R}^{n}$ :

$$
\left\{\begin{array}{l}
y_{n}=P_{C}\left(x_{n}-\tau A x_{n}\right), \\
x_{n+1}=P_{C}\left(x_{n}-\tau A y_{n}\right), \quad \forall n \geq 0,
\end{array}\right.
$$

with $\tau>0$ a given number, which is known as the extragradient method. The literature on the VIP is vast and Korpelevich's extragradient method has received great attention given by many authors, who improved it in various ways; see e.g., [5-20] and the references therein.

On the other hand, we consider the following general mixed equilibrium problem (GMEP) (see, also, $[21,22]$ ) of finding $x \in C$ such that

$$
\Theta(x, y)+h(x, y) \geq 0, \quad \forall y \in C,
$$

where $\Theta, h: C \times C \rightarrow \mathbf{R}$ are two bifunctions. We denote the set of solutions of GMEP (1.2) by $\operatorname{GMEP}(\Theta, h)$. The GMEP $(1.2)$ is very general; for example, it includes the following equilibrium problems as special cases.

As an example, in $[12,23,24]$ the authors considered and studied the generalized equilibrium problem (GEP), which is to find $x \in C$ such that

$$
\Theta(x, y)+\langle A x, y-x\rangle \geq 0, \quad \forall y \in C .
$$

The set of solutions of GEP is denoted by $\operatorname{GEP}(\Theta, A)$.

In $[21,25,26]$, the authors considered and studied the mixed equilibrium problem (MEP), which is to find $x \in C$ such that

$$
\Theta(x, y)+\varphi(y)-\varphi(x) \geq 0, \quad \forall y \in C .
$$

The set of solutions of MEP is denoted by $\operatorname{MEP}(\Theta, \varphi)$.

In [27-30], the authors considered and studied the equilibrium problem (EP), which is to find $x \in C$ such that

$$
\Theta(x, y) \geq 0, \quad \forall y \in C .
$$

The set of solutions of EP is denoted by $\operatorname{EP}(\Theta)$. It is worth to mention that the EP is an unified model of several problems, namely, variational inequality problems, optimization problems, saddle point problems, complementarity problems, fixed point problems, Nash equilibrium problems, etc. 
Throughout this paper, it is assumed as in [31] that $\Theta: C \times C \rightarrow \mathbf{R}$ is a bifunction satisfying conditions ( $\theta 1)-(\theta 3)$ and $h: C \times C \rightarrow \mathbf{R}$ is a bifunction with restrictions (h1)-(h3), where

$(\theta 1) \Theta(x, x)=0$ for all $x \in C$;

$(\theta 2) \Theta$ is monotone (i.e., $\Theta(x, y)+\Theta(y, x) \leq 0, \forall x, y \in C)$ and upper hemicontinuous in the first variable, i.e., for each $x, y, z \in C$,

$$
\limsup _{t \rightarrow 0^{+}} \Theta(t z+(1-t) x, y) \leq \Theta(x, y)
$$

$(\theta 3) \Theta$ is lower semicontinuous and convex in the second variable;

(h1) $h(x, x)=0$ for all $x \in C$;

(h2) $h$ is monotone and weakly upper semicontinuous in the first variable;

(h3) $h$ is convex in the second variable.

For $r>0$ and $x \in H$, let $T_{r}: H \rightarrow 2^{C}$ be a mapping defined by

$$
T_{r} x=\left\{z \in C: \Theta(z, y)+h(z, y)+\frac{1}{r}\langle y-z, z-x\rangle \geq 0, \forall y \in C\right\},
$$

called the resolvent of $\Theta$ and $h$.

Let $\left\{T_{n}\right\}_{n=1}^{\infty}$ be an infinite family of nonexpansive self-mappings on $C$ and $\left\{\lambda_{n}\right\}_{n=1}^{\infty}$ be a sequence of nonnegative numbers in $[0,1]$. For any $n \geq 1$, define a mapping $W_{n}$ on $C$ as follows:

$$
\left\{\begin{array}{l}
U_{n, n+1}=I \\
U_{n, n}=\lambda_{n} T_{n} U_{n, n+1}+\left(1-\lambda_{n}\right) I \\
U_{n, n-1}=\lambda_{n-1} T_{n-1} U_{n, n}+\left(1-\lambda_{n-1}\right) I \\
\cdots \\
U_{n, k}=\lambda_{k} T_{k} U_{n, k+1}+\left(1-\lambda_{k}\right) I \\
U_{n, k-1}=\lambda_{k-1} T_{k-1} U_{n, k}+\left(1-\lambda_{k-1}\right) I \\
\cdots \\
U_{n, 2}=\lambda_{2} T_{2} U_{n, 3}+\left(1-\lambda_{2}\right) I \\
W_{n}=U_{n, 1}=\lambda_{1} T_{1} U_{n, 2}+\left(1-\lambda_{1}\right) I
\end{array}\right.
$$

Such a mapping $W_{n}$ is called the $W$-mapping generated by $T_{n}, T_{n-1}, \ldots, T_{1}$ and $\lambda_{n}, \lambda_{n-1}$, $\ldots, \lambda_{1}$.

In 2013, Rattanaseeha [30] introduced an iterative algorithm:

$$
\left\{\begin{array}{l}
x_{1} \in H \text { arbitrarily given, } \\
\Theta\left(u_{n}, y\right)+\frac{1}{r_{n}}\left\langle y-u_{n}, u_{n}-x_{n}\right\rangle \geq 0, \quad \forall y \in C \\
x_{n+1}=P_{C}\left[\alpha_{n} \gamma f\left(x_{n}\right)+\left(I-\alpha_{n} V\right) W_{n} u_{n}\right], \quad \forall n \geq 1
\end{array}\right.
$$

and proved the following strong convergence theorem.

Theorem $\mathbf{R}$ (see [30], Theorem 3.1) Let $C$ be a nonempty closed convex subset of a real Hilbert space $H$. Let $\Theta: C \times C \rightarrow \mathbf{R}$ be a bifunction satisfying assumptions (A1)-(A4). Let $f$ be an $\alpha$-contraction on $H$ with $\alpha \in(0,1)$, and let $\left\{T_{n}\right\}_{n=1}^{\infty}$ be an infinite family of nonexpansive self-mappings on $C$ such that $\Omega:=\bigcap_{n=1}^{\infty} \operatorname{Fix}\left(T_{n}\right) \cap \operatorname{EP}(\Theta) \neq \emptyset$. Let $V: H \rightarrow H$ be 
a $\bar{\gamma}$-strongly positive bounded linear operator with $0<\gamma<\frac{\bar{\gamma}}{\alpha}$. Let $\lambda_{1}, \lambda_{2}, \ldots$ be a sequence of real numbers such that $0<\lambda_{n} \leq b<1, n=1,2, \ldots$. Let $W_{n}$ be the $W$-mapping of $C$ into itself generated by (1.4). Let $W$ be defined by $W x=\lim _{n \rightarrow \infty} W_{n} x, \forall x \in C$. Let $\left\{x_{n}\right\}$ and $\left\{u_{n}\right\}$ be sequences generated by $(1.5)$, where $\left\{\alpha_{n}\right\}$ is a sequence in $(0,1)$ and $\left\{r_{n}\right\}$ is a sequence in $(0, \infty)$ such that the following conditions hold:

(C1) $\lim _{n \rightarrow \infty} \alpha_{n}=0$,

(C2) $\sum_{n=1}^{\infty} \alpha_{n}=\infty$, and

(C3) $\lim _{n \rightarrow \infty} r_{n}=r>0$.

Then both $\left\{x_{n}\right\}$ and $\left\{u_{n}\right\}$ converge strongly to $x^{*} \in \Omega$, where $x^{*}=P_{\Omega}(I-(V-\gamma f)) x^{*}$ is a unique solution of the VIP

$$
\left\langle(V-\gamma f) x^{*}, x^{*}-x\right\rangle \leq 0, \quad \forall x \in \Omega,
$$

or, equivalently, the unique solution of the minimization problem

$$
\min _{x \in \Omega} \frac{1}{2}\langle V x, x\rangle-\Psi(x)
$$

where $\Psi$ is a potential function for $\gamma f$.

Let $F_{1}, F_{2}: C \rightarrow H$ be two mappings. Consider the general system of variational inequalities (GSVI) of finding $\left(x^{*}, y^{*}\right) \in C \times C$ such that

$$
\begin{cases}\left\langle v_{1} F_{1} y^{*}+x^{*}-y^{*}, x-x^{*}\right\rangle \geq 0, & \forall x \in C, \\ \left\langle v_{2} F_{2} x^{*}+y^{*}-x^{*}, x-y^{*}\right\rangle \geq 0, & \forall x \in C,\end{cases}
$$

where $v_{1}>0$ and $v_{2}>0$ are two constants. The solution set of GSVI (1.6) is denoted by $\operatorname{GSVI}\left(C, F_{1}, F_{2}\right)$.

In particular, if $F_{1}=F_{2}=A$, then the GSVI (1.6) reduces to the problem of finding $\left(x^{*}, y^{*}\right) \in C \times C$ such that

$$
\begin{cases}\left\langle v_{1} A y^{*}+x^{*}-y^{*}, x-x^{*}\right\rangle \geq 0, & \forall x \in C, \\ \left\langle v_{2} A x^{*}+y^{*}-x^{*}, x-y^{*}\right\rangle \geq 0, & \forall x \in C,\end{cases}
$$

which is defined by Verma [32] and it is called a new system of variational inequalities (NSVI). Further, if $x^{*}=y^{*}$ additionally, then the NSVI reduces to the classical VIP (1.1). In 2008, Ceng et al. [8] transformed the GSVI (1.6) into the fixed point problem of the mapping $G=P_{C}\left(I-v_{1} F_{1}\right) P_{C}\left(I-v_{2} F_{2}\right)$, that is, $G x^{*}=x^{*}$, where $y^{*}=P_{C}\left(I-v_{2} F_{2}\right) x^{*}$. Throughout this paper, the fixed point set of the mapping $G$ is denoted by $\Xi$.

In 2012, Marino et al. [33] introduced a multi-step iterative scheme

$$
\left\{\begin{array}{l}
\Theta\left(u_{n}, y\right)+h\left(u_{n}, y\right)+\frac{1}{r_{n}}\left\langle y-u_{n}, u_{n}-x_{n}\right\rangle \geq 0, \quad \forall y \in C, \\
y_{n, 1}=\beta_{n, 1} S_{1} u_{n}+\left(1-\beta_{n, 1}\right) u_{n} \\
y_{n, i}=\beta_{n, i} S_{i} u_{n}+\left(1-\beta_{n, i}\right) y_{n, i-1}, \quad i=2, \ldots, N \\
x_{n+1}=\alpha_{n} f\left(x_{n}\right)+\left(1-\alpha_{n}\right) T y_{n, N},
\end{array}\right.
$$

with $f: C \rightarrow C$ a $\rho$-contraction and $\left\{\alpha_{n}\right\},\left\{\beta_{n, i}\right\} \subset(0,1),\left\{r_{n}\right\} \subset(0, \infty)$, which generalizes the two-step iterative scheme in [34] for two nonexpansive mappings to a finite family of 
nonexpansive mappings $T, S_{i}: C \rightarrow C, i=1, \ldots, N$, and proved that the proposed scheme (1.7) converges strongly to a common fixed point of the mappings that is also an equilibrium point of the GMEP (1.2).

More recently, Marino et al.'s multi-step iterative scheme (1.7) was extended to develop the following relaxed viscosity iterative algorithm by virtue of Korpelevich's extragradient method.

Algorithm CKW (see (3.1) in [13]) Let $f: C \rightarrow C$ be a $\rho$-contraction and $T: C \rightarrow C$ be a $\xi$-strict pseudocontraction. Let $S_{i}: C \rightarrow C$ be a nonexpansive mapping for each $i=$ $1, \ldots, N$. Let $F_{j}: C \rightarrow H$ be $\zeta_{j}$-inverse strongly monotone with $0<\nu_{j}<\zeta_{j}$ for each $j=1,2$. Let $\Theta: C \times C \rightarrow \mathbf{R}$ be a bifunction satisfying conditions $(\theta 1)-(\theta 3)$ and $h: C \times C \rightarrow \mathbf{R}$ be a bifunction with restrictions (h1)-(h3). Let $\left\{x_{n}\right\}$ be the sequence generated by

$$
\left\{\begin{array}{l}
\Theta\left(u_{n}, y\right)+h\left(u_{n}, y\right)+\frac{1}{r_{n}}\left\langle y-u_{n}, u_{n}-x_{n}\right\rangle \geq 0, \quad \forall y \in C, \\
y_{n, 1}=\beta_{n, 1} S_{1} u_{n}+\left(1-\beta_{n, 1}\right) u_{n}, \\
y_{n, i}=\beta_{n, i} S_{i} u_{n}+\left(1-\beta_{n, i}\right) y_{n, i-1}, \quad i=2, \ldots, N, \\
y_{n}=\alpha_{n} f\left(y_{n, N}\right)+\left(1-\alpha_{n}\right) G y_{n, N} \\
x_{n+1}=\beta_{n} x_{n}+\gamma_{n} y_{n}+\delta_{n} T y_{n}, \quad \forall n \geq 0,
\end{array}\right.
$$

where $G=P_{C}\left(I-v_{1} F_{1}\right) P_{C}\left(I-v_{2} F_{2}\right),\left\{\alpha_{n}\right\},\left\{\beta_{n}\right\}$ are sequences in $(0,1)$ with

$$
0<\liminf _{n \rightarrow \infty} \beta_{n} \leq \limsup _{n \rightarrow \infty} \beta_{n}<1,
$$

$\left\{\gamma_{n}\right\},\left\{\delta_{n}\right\}$ are sequences in $[0,1]$ with $\liminf _{n \rightarrow \infty} \delta_{n}>0$ and $\beta_{n}+\gamma_{n}+\delta_{n}=1, \forall n \geq 0,\left\{\beta_{n, i}\right\}$ is a sequence in $(0,1)$ for each $i=1, \ldots, N,\left(\gamma_{n}+\delta_{n}\right) \xi \leq \gamma_{n}, \forall n \geq 0$, and $\left\{r_{n}\right\}$ is a sequence in $(0, \infty)$ with $\liminf _{n \rightarrow \infty} r_{n}>0$.

The authors [13] proved that the proposed scheme (1.8) converges strongly to a common fixed point of the mappings $T, S_{i}: C \rightarrow C, i=1, \ldots, N$, which is also an equilibrium point of the GMEP (1.2) and a solution of the GSVI (1.6).

Furthermore, let $B$ be a single-valued mapping of $C$ into $H$ and $R$ be a multivalued mapping with $D(R)=C$. Consider the following variational inclusion: find a point $x \in C$ such that

$$
0 \in B x+R x
$$

We denote by $\mathrm{I}(B, R)$ the solution set of the variational inclusion (1.9). In particular, if $B=R=0$, then $\mathrm{I}(B, R)=C$. If $B=0$, then problem (1.9) becomes the inclusion problem introduced by Rockafellar [35]. It is known that problem (1.9) provides a convenient framework for the unified study of optimal solutions in many optimization related areas including mathematical programming, complementarity problems, variational inequalities, optimal control, mathematical economics, equilibria, game theory, etc. Let a set-valued mapping $R: D(R) \subset H \rightarrow 2^{H}$ be maximal monotone. We define the resolvent operator $J_{R, \lambda}: H \rightarrow \overline{D(R)}$ associated with $R$ and $\lambda$ as follows:

$$
J_{R, \lambda}=(I+\lambda R)^{-1}, \quad \forall x \in H,
$$

where $\lambda$ is a positive number. 
In 1998, Huang [36] studied problem (1.9) in the case where $R$ is maximal monotone and $B$ is strongly monotone and Lipschitz-continuous with $D(R)=C=H$. Subsequently, Zeng et al. [37] further studied problem (1.9) in the case which is more general than Huang's one [36]. Moreover, the authors [37] obtained the same strong convergence conclusion as in Huang's result [36]. In addition, the authors also gave the geometric convergence rate estimate for approximate solutions. Also, various types of iterative algorithms for solving variational inclusions have been further studied and developed; for more details, refer to $[11,15,38-40]$ and the references therein.

Very recently, Ceng et al. [41] introduced and analyzed one multi-step hybrid steepestdescent extragradient algorithm and another multi-step composite Mann-type viscosity iterative algorithm for finding a solution of triple hierarchical variational inequalities defined over the common set of solutions of finitely many generalized mixed equilibrium problems, finitely many variational inclusions, a general system of variational inequalities, and a fixed point problem of a strict pseudocontraction in a real Hilbert space $H$. Here, the generalized mixed equilibrium problem is defined as follows: Let $\varphi: C \rightarrow \mathbf{R}$ be a realvalued function, $A: C \rightarrow H$ be a nonlinear mapping and $\Theta: C \times C \rightarrow \mathbf{R}$ be a bifunction. Then the objective is to find $x \in C$ such that

$$
\Theta(x, y)+\varphi(y)-\varphi(x)+\langle A x, y-x\rangle \geq 0, \quad \forall y \in C .
$$

The solution set of such generalized mixed equilibrium problem is denoted by $\operatorname{GMEP}(\Theta$, $\varphi, A)$. Under appropriate assumptions, the authors proved that the proposed algorithms converge strongly to an element of the common set, which is a unique solution of a triple hierarchical variational inequality problem; see [41], Theorems 3.1 and 4.1.

In this paper, we introduce a relaxed extragradient iterative algorithm for finding a common element of the solution set $\operatorname{GMEP}(\Theta, h)$ of $\operatorname{GMEP}(1.2)$, the solution set $\operatorname{GSVI}\left(C, F_{1}, F_{2}\right)($ i.e., $\Xi)$ of GSVI (1.6), the solution set $\bigcap_{k=1}^{M} \mathrm{I}\left(B_{k}, R_{k}\right)$ of finitely many variational inclusions for maximal monotone mappings $\left\{R_{k}\right\}_{k=1}^{M}$ and inverse-strongly monotone mappings $\left\{B_{k}\right\}_{k=1}^{M}$, and the common fixed point set $\bigcap_{i=1}^{N} \operatorname{Fix}\left(S_{i}\right) \cap \operatorname{Fix}(T)$ of finitely many nonexpansive mappings $S_{i}: C \rightarrow C, i=1, \ldots, N$, and a strictly pseudocontractive mapping $T: H \rightarrow H$, in the setting of the infinite-dimensional Hilbert space. The iterative algorithm is based on Korpelevich's extragradient method, viscosity approximation method [42] (see also [43]), Mann's iterative method, and strongly positive bounded linear operator approach. Our aim is to prove that the iterative algorithm converges strongly to a common element of these sets, which also solves some hierarchical minimization. We observe that related results have been derived say in [13, 26, 28, 29, 33, 34, 44-51]. In addition, we also point out what are different between the present article and the previous one [41] as follows:

(i) The problem of finding an element of $\operatorname{Fix}(T) \cap \bigcap_{i=1}^{N} \operatorname{Fix}\left(S_{i}\right) \cap \operatorname{GMEP}(\Theta, h) \cap \bigcap_{k=1}^{M} \mathrm{I}\left(B_{k}\right.$, $\left.R_{k}\right) \cap \Xi$ in Theorems 3.1 and 3.2 of this paper is very different from the one of finding an element of $\bigcap_{k=1}^{M} \operatorname{GMEP}\left(\Theta_{k}, \varphi_{k}, A_{k}\right) \cap \bigcap_{i=1}^{N} \mathrm{I}\left(B_{i}, R_{i}\right) \cap \Xi \cap \operatorname{Fix}(T)$ in [41], Theorems 3.1 and 4.1, where $S_{i}$ is a nonexpansive mapping for each $i \in\{1, \ldots, N\}$ and $T$ is a strict pseudocontraction. It is clear that the general mixed equilibrium problem (1.2) is very different from the above generalized mixed equilibrium problem.

(ii) The iterative scheme (3.1) in this paper is very different from the iterative schemes (3.1) and (4.1) in the authors [41] because the scheme (3.1) involves finding a common fixed point of finitely many nonexpansive mappings $\left\{S_{i}\right\}_{i=1}^{N}$ and a strict pseudocon- 
traction $T$. In the meantime, Theorems 3.1 and 3.2 of this paper show that the proposed algorithm converges strongly to a unique solution of a VIP defined over the set $\operatorname{Fix}(T) \cap \bigcap_{i=1}^{N} \operatorname{Fix}\left(S_{i}\right) \cap \operatorname{GMEP}(\Theta, h) \cap \bigcap_{k=1}^{M} \mathrm{I}\left(B_{k}, R_{k}\right) \cap \Xi$. However, Theorems 3.1 and 4.1 in [41] show that the proposed algorithms converge strongly to a unique solution of a triple hierarchical variational inequality problem defined over the set $\bigcap_{k=1}^{M} \operatorname{GMEP}\left(\Theta_{k}, \varphi_{k}, A_{k}\right) \cap$ $\bigcap_{i=1}^{N} \mathrm{I}\left(B_{i}, R_{i}\right) \cap \Xi \cap \operatorname{Fix}(T)$.

\section{Preliminaries}

Throughout this paper, we assume that $H$ is a real Hilbert space whose inner product and norm are denoted by $\langle\cdot, \cdot\rangle$ and $\|\cdot\|$, respectively. Let $C$ be a nonempty closed convex subset of $H$. We write $x_{n} \rightarrow x$ to indicate that the sequence $\left\{x_{n}\right\}$ converges weakly to $x$ and $x_{n} \rightarrow x$ to indicate that the sequence $\left\{x_{n}\right\}$ converges strongly to $x$. Moreover, we use $\omega_{w}\left(x_{n}\right)$ to denote the weak $\omega$-limit set of the sequence $\left\{x_{n}\right\}$ and $\omega_{s}\left(x_{n}\right)$ to denote the strong $\omega$-limit set of the sequence $\left\{x_{n}\right\}$, i.e.,

$$
\omega_{w}\left(x_{n}\right):=\left\{x \in H: x_{n_{i}} \rightarrow x \text { for some subsequence }\left\{x_{n_{i}}\right\} \text { of }\left\{x_{n}\right\}\right\},
$$

and

$$
\omega_{s}\left(x_{n}\right):=\left\{x \in H: x_{n_{i}} \rightarrow x \text { for some subsequence }\left\{x_{n_{i}}\right\} \text { of }\left\{x_{n}\right\}\right\} .
$$

The metric (or nearest point) projection from $H$ onto $C$ is the mapping $P_{C}: H \rightarrow C$ which assigns to each point $x \in H$ the unique point $P_{C} x \in C$ satisfying the property

$$
\left\|x-P_{C} x\right\|=\inf _{y \in C}\|x-y\|=: d(x, C) .
$$

The following properties of projections are useful and pertinent to our purpose.

Proposition 2.1 Given any $x \in H$ and $z \in C$. One has

(i) $z=P_{C} x \Leftrightarrow\langle x-z, y-z\rangle \leq 0, \forall y \in C$;

(ii) $z=P_{C} x \Leftrightarrow\|x-z\|^{2} \leq\|x-y\|^{2}-\|y-z\|^{2}, \forall y \in C$;

(iii) $\left\langle P_{C} x-P_{C} y, x-y\right\rangle \geq\left\|P_{C} x-P_{C} y\right\|^{2}, \forall y \in H$, which hence implies that $P_{C}$ is nonexpansive and monotone.

Definition 2.1 A mapping $T: H \rightarrow H$ is said to be

(a) nonexpansive if

$$
\|T x-T y\| \leq\|x-y\|, \quad \forall x, y \in H
$$

(b) firmly nonexpansive if $2 T-I$ is nonexpansive, or equivalently, if $T$ is 1-inverse-strongly monotone (1-ism),

$$
\langle x-y, T x-T y\rangle \geq\|T x-T y\|^{2}, \quad \forall x, y \in H ;
$$

alternatively, $T$ is firmly nonexpansive if and only if $T$ can be expressed as

$$
T=\frac{1}{2}(I+S)
$$

where $S: H \rightarrow H$ is nonexpansive; projections are firmly nonexpansive. 
Definition 2.2 A mapping $A: C \rightarrow H$ is said to be

(i) monotone if

$$
\langle A x-A y, x-y\rangle \geq 0, \quad \forall x, y \in C
$$

(ii) $\eta$-strongly monotone if there exists a constant $\eta>0$ such that

$$
\langle A x-A y, x-y\rangle \geq \eta\|x-y\|^{2}, \quad \forall x, y \in C
$$

(iii) $\zeta$-inverse-strongly monotone if there exists a constant $\zeta>0$ such that

$$
\langle A x-A y, x-y\rangle \geq \zeta\|A x-A y\|^{2}, \quad \forall x, y \in C .
$$

It can easily be seen that if $T$ is nonexpansive, then $I-T$ is monotone. It is also easy to see that the projection $P_{C}$ is 1-ism. Inverse-strongly monotone (also referred to as co-coercive) operators have been applied widely in solving practical problems in various fields.

On the other hand, it is obvious that if $A: C \rightarrow H$ is $\zeta$-inverse-strongly monotone, then $A$ is monotone and $\frac{1}{\zeta}$-Lipschitz-continuous. Moreover, we also note that, for all $u, v \in C$ and $\lambda>0$,

$$
\|(I-\lambda A) u-(I-\lambda A) v\|^{2} \leq\|u-v\|^{2}+\lambda(\lambda-2 \zeta)\|A u-A v\|^{2} .
$$

So, if $\lambda \leq 2 \zeta$, then $I-\lambda A$ is a nonexpansive mapping from $C$ to $H$.

In 2008, Ceng et al. [8] transformed problem (1.6) into a fixed point problem in the following way.

Proposition 2.2 (see [8]) For given $\bar{x}, \bar{y} \in C,(\bar{x}, \bar{y})$ is a solution of the GSVI (1.6) if and only if $\bar{x}$ is a fixed point of the mapping $G: C \rightarrow C$ defined by

$$
G x=P_{C}\left(I-v_{1} F_{1}\right) P_{C}\left(I-v_{2} F_{2}\right) x, \quad \forall x \in C,
$$

where $\bar{y}=P_{C}\left(I-v_{2} F_{2}\right) \bar{x}$.

In particular, if the mapping $F_{j}: C \rightarrow H$ is $\zeta_{j}$-inverse-strongly monotone for $j=1,2$, then the mapping $G$ is nonexpansive provided $v_{j} \in\left(0,2 \zeta_{j}\right]$ for $j=1,2$. We denote by $\Xi$ denote the fixed point set of the mapping $G$.

We need some facts and tools in a real Hilbert space $H$ which are listed as lemmas below.

Lemma 2.1 Let $X$ be a real inner product space. Then we have the following inequality:

$$
\|x+y\|^{2} \leq\|x\|^{2}+2\langle y, x+y\rangle, \quad \forall x, y \in X .
$$

Lemma 2.2 Let $H$ be a real Hilbert space. Then the following hold:

(a) $\|x-y\|^{2}=\|x\|^{2}-\|y\|^{2}-2\langle x-y, y\rangle$ for all $x, y \in H$;

(b) $\|\lambda x+\mu y\|^{2}=\lambda\|x\|^{2}+\mu\|y\|^{2}-\lambda \mu\|x-y\|^{2}$ for all $x, y \in H$ and $\lambda, \mu \in[0,1]$ with $\lambda+\mu=1$ 
(c) if $\left\{x_{n}\right\}$ is a sequence in $H$ such that $x_{n} \rightarrow x$, it follows that

$$
\limsup _{n \rightarrow \infty}\left\|x_{n}-y\right\|^{2}=\limsup _{n \rightarrow \infty}\left\|x_{n}-x\right\|^{2}+\|x-y\|^{2}, \quad \forall y \in H .
$$

It is clear that, in a real Hilbert space $H, T: C \rightarrow C$ is $\xi$-strictly pseudocontractive if and only if the following inequality holds:

$$
\langle T x-T y, x-y\rangle \leq\|x-y\|^{2}-\frac{1-\xi}{2}\|(I-T) x-(I-T) y\|^{2}, \quad \forall x, y \in C .
$$

This immediately implies that if $T$ is a $\xi$-strictly pseudocontractive mapping, then $I-T$ is $\frac{1-\xi}{2}$-inverse strongly monotone; for further details, we refer to [52] and the references therein. It is well known that the class of strict pseudocontractions strictly includes the class of nonexpansive mappings and that the class of pseudocontractions strictly includes the class of strict pseudocontractions.

Lemma 2.3 (see [52], Proposition 2.1) Let $C$ be a nonempty closed convex subset of a real Hilbert space $H$ and $T: C \rightarrow C$ be a mapping.

(i) If $T$ is a $\xi$-strictly pseudocontractive mapping, then $T$ satisfies the Lipschitzian condition

$$
\|T x-T y\| \leq \frac{1+\xi}{1-\xi}\|x-y\|, \quad \forall x, y \in C .
$$

(ii) If $T$ is a $\xi$-strictly pseudocontractive mapping, then the mapping $I-T$ is semiclosed at 0 , that is, if $\left\{x_{n}\right\}$ is a sequence in $C$ such that $x_{n}-\tilde{x}$ and $(I-T) x_{n} \rightarrow 0$, then $(I-T) \tilde{x}=0$.

(iii) If $T$ is $\xi$-(quasi-)strict pseudocontraction, then the fixed point set $\operatorname{Fix}(T)$ of $T$ is closed and convex so that the projection $P_{\operatorname{Fix}(T)}$ is well defined.

Lemma 2.4 (see [14]) Let $C$ be a nonempty closed convex subset of a real Hilbert space $H$. Let $T: C \rightarrow C$ be a $\xi$-strictly pseudocontractive mapping. Let $\gamma$ and $\delta$ be two nonnegative real numbers such that $(\gamma+\delta) \xi \leq \gamma$. Then

$$
\|\gamma(x-y)+\delta(T x-T y)\| \leq(\gamma+\delta)\|x-y\|, \quad \forall x, y \in C .
$$

Lemma 2.5 (see [53], demiclosedness principle) Let C be a nonempty closed convex subset of a real Hilbert space $H$. Let $S$ be a nonexpansive self-mapping on $C$. Then $I-S$ is demiclosed. That is, whenever $\left\{x_{n}\right\}$ is a sequence in $C$ weakly converging to some $x \in C$ and the sequence $\left\{(I-S) x_{n}\right\}$ strongly converges to some $y$, it follows that $(I-S) x=y$. Here $I$ is the identity operator of $H$.

Lemma 2.6 Let $A: C \rightarrow H$ be a monotone mapping. In the context of the variational inequality problem the characterization of the projection (see Proposition 2.1(i)) implies

$$
u \in \operatorname{VI}(C, A) \quad \Leftrightarrow \quad u=P_{C}(u-\lambda A u), \quad \lambda>0 .
$$

Lemma 2.7 (see [54]) Let $V$ be a $\bar{\gamma}$-strongly positive bounded linear operator on $H$ and assume $0<\rho \leq\|V\|^{-1}$. Then $\|I-\rho V\| \leq 1-\rho \bar{\gamma}$. 
Lemma 2.8 (see [55]) Let $\left\{a_{n}\right\}$ be a sequence of nonnegative real numbers satisfying

$$
a_{n+1} \leq\left(1-s_{n}\right) a_{n}+s_{n} b_{n}+t_{n}, \quad \forall n \geq 1,
$$

where $\left\{s_{n}\right\},\left\{t_{n}\right\}$, and $\left\{b_{n}\right\}$ satisfy the following conditions:

(i) $\left\{s_{n}\right\} \subset[0,1]$ and $\sum_{n=1}^{\infty} s_{n}=\infty$;

(ii) either $\limsup _{n \rightarrow \infty} b_{n} \leq 0$ or $\sum_{n=1}^{\infty}\left|s_{n} b_{n}\right|<\infty$;

(iii) $t_{n} \geq 0$ for all $n \geq 1$, and $\sum_{n=1}^{\infty} t_{n}<\infty$.

Then $\lim _{n \rightarrow \infty} a_{n}=0$.

In the sequel, we will indicate with $\operatorname{GMEP}(\Theta, h)$ the solution set of GMEP (1.2).

Lemma 2.9 (see [31]) Let $C$ be a nonempty closed convex subset of a real Hilbert space $H$. Let $\Theta: C \times C \rightarrow \mathbf{R}$ be a bifunction satisfying conditions $(\theta 1)-(\theta 3)$ and $h: C \times C \rightarrow \mathbf{R}$ is a bifunction with restrictions (h1)-(h3). Moreover, let us suppose that

(H) for fixed $r>0$ and $x \in C$, there exist a bounded $K \subset C$ and $\hat{x} \in K$ such that for all $z \in C \backslash K,-\Theta(\hat{x}, z)+h(z, \hat{x})+\frac{1}{r}\langle\hat{x}-z, z-x\rangle<0$.

For $r>0$ and $x \in H$, the mapping $T_{r}: H \rightarrow 2^{C}$ (i.e., the resolvent of $\Theta$ and $h$ ) has the following properties:

(i) $T_{r} x \neq \emptyset$;

(ii) $T_{r} x$ is a singleton;

(iii) $T_{r}$ is firmly nonexpansive;

(iv) $\operatorname{GMEP}(\Theta, h)=\operatorname{Fix}\left(T_{r}\right)$ and it is closed and convex.

Lemma 2.10 (see [31]) Let us suppose that ( $(\theta 1)-(\theta 3),(\mathrm{h} 1)-(\mathrm{h} 3)$, and $(\mathrm{H})$ hold. Let $x, y \in H$, $r_{1}, r_{2}>0$. Then

$$
\left\|T_{r_{2}} y-T_{r_{1}} x\right\| \leq\|y-x\|+\left|\frac{r_{2}-r_{1}}{r_{2}}\right|\left\|T_{r_{2}} y-y\right\| .
$$

Lemma 2.11 (see [33]) Suppose that the hypotheses of Lemma 2.9 are satisfied. Let $\left\{r_{n}\right\}$ be a sequence in $(0, \infty)$ with $\liminf _{n \rightarrow \infty} r_{n}>0$. Suppose that $\left\{x_{n}\right\}$ is a bounded sequence. Then the following statements are equivalent and true:

(a) if $\left\|x_{n}-T_{r_{n}} x_{n}\right\| \rightarrow 0$ as $n \rightarrow \infty$, each weak cluster point of $\left\{x_{n}\right\}$ satisfies the problem

$$
\begin{aligned}
\Theta(x, y)+h(x, y) \geq 0, \quad \forall y \in C, \\
\text { i.e., } \omega_{w}\left(x_{n}\right) \subseteq \operatorname{GMEP}(\Theta, h) ;
\end{aligned}
$$

(b) the demiclosedness principle holds in the sense that, if $x_{n} \rightarrow x^{*}$ and $\left\|x_{n}-T_{r_{n}} x_{n}\right\| \rightarrow 0$ as $n \rightarrow \infty$, then $\left(I-T_{r_{k}}\right) x^{*}=0$ for all $k \geq 1$.

Recall that a set-valued mapping $T: D(T) \subset H \rightarrow 2^{H}$ is called monotone if for all $x, y \in$ $D(T), f \in T x$ and $g \in T y$ imply

$$
\langle f-g, x-y\rangle \geq 0
$$

A set-valued mapping $T$ is called maximal monotone if $T$ is monotone and $(I+\lambda T) D(T)=$ $H$ for each $\lambda>0$, where $I$ is the identity mapping of $H$. We denote by $G(T)$ the graph of $T$. 
It is well known that a monotone mapping $T$ is maximal if and only if, for $(x, f) \in H \times H$, $\langle f-g, x-y\rangle \geq 0$ for every $(y, g) \in G(T)$ implies $f \in T x$. Next we provide an example to illustrate the concept of a maximal monotone mapping.

Let $A: C \rightarrow H$ be a monotone, $k$-Lipschitz-continuous mapping and let $N_{C} v$ be the normal cone to $C$ at $v \in C$, i.e.,

$$
N_{C} v=\{u \in H:\langle v-p, u\rangle \geq 0, \forall p \in C\} .
$$

Define

$$
\widetilde{T} v= \begin{cases}A v+N_{C} v, & \text { if } v \in C, \\ \emptyset, & \text { if } v \notin C .\end{cases}
$$

Then it is well known [4] that $\widetilde{T}$ is maximal monotone and $0 \in \widetilde{T} v$ if and only if $v \in$ $\mathrm{VI}(C, A)$.

Let $R: D(R) \subset H \rightarrow 2^{H}$ be a maximal monotone mapping. Let $\lambda, \mu>0$ be two positive numbers.

Lemma 2.12 (see [56]) We have the resolvent identity

$$
J_{R, \lambda} x=J_{R, \mu}\left(\frac{\mu}{\lambda} x+\left(1-\frac{\mu}{\lambda}\right) J_{R, \lambda} x\right), \quad \forall x \in H
$$

Remark 2.1 For $\lambda, \mu>0$, we have the following relation:

$$
\left\|J_{R, \lambda} x-J_{R, \mu} y\right\| \leq\|x-y\|+|\lambda-\mu|\left(\frac{1}{\lambda}\left\|J_{R, \lambda} x-y\right\|+\frac{1}{\mu}\left\|x-J_{R, \mu} y\right\|\right), \quad \forall x, y \in H .
$$

In terms of Huang [36] (see also [37]), we have the following property for the resolvent operator $J_{R, \lambda}: H \rightarrow \overline{D(R)}$.

Lemma 2.13 $J_{R, \lambda}$ is single-valued and firmly nonexpansive, i.e.,

$$
\left\langle J_{R, \lambda} x-J_{R, \lambda} y, x-y\right\rangle \geq\left\|J_{R, \lambda} x-J_{R, \lambda} y\right\|^{2}, \quad \forall x, y \in H .
$$

Consequently, $J_{R, \lambda}$ is nonexpansive and monotone.

Lemma 2.14 (see [15]) Let $R$ be a maximal monotone mapping with $D(R)=C$. Then for any given $\lambda>0, u \in C$ is a solution of problem (1.9) if and only if $u \in C$ satisfies

$$
u=J_{R, \lambda}(u-\lambda B u) .
$$

Lemma 2.15 (see [37]) Let $R$ be a maximal monotone mapping with $D(R)=C$ and let $B: C \rightarrow H$ be a strongly monotone, continuous, and single-valued mapping. Then for each $z \in H$, the equation $z \in(B+\lambda R) x$ has a unique solution $x_{\lambda}$ for $\lambda>0$.

Lemma 2.16 (see [15]) Let $R$ be a maximal monotone mapping with $D(R)=C$ and $B$ : $C \rightarrow H$ be a monotone, continuous and single-valued mapping. Then $(I+\lambda(R+B)) C=H$ for each $\lambda>0$. In this case, $R+B$ is maximal monotone. 


\section{Main results}

We now propose the following relaxed extragradient iterative scheme:

$$
\left\{\begin{array}{l}
\Theta\left(u_{n}, y\right)+h\left(u_{n}, y\right)+\frac{1}{r_{n}}\left\langle y-u_{n}, u_{n}-x_{n}\right\rangle \geq 0, \quad \forall y \in C, \\
v_{n}=J_{R_{M}, \lambda_{M, n}}\left(I-\lambda_{M, n} B_{M}\right) J_{R_{M-1}, \lambda_{M-1, n}}\left(I-\lambda_{M-1, n} B_{M-1}\right) \cdots J_{R_{1}, \lambda_{1, n}}\left(I-\lambda_{1, n} B_{1}\right) u_{n}, \\
y_{n, 1}=\beta_{n, 1} S_{1} v_{n}+\left(1-\beta_{n, 1}\right) v_{n}, \\
y_{n, i}=\beta_{n, i} S_{i} v_{n}+\left(1-\beta_{n, i}\right) y_{n, i-1}, \quad i=2, \ldots, N, \\
y_{n}=\alpha_{n} \gamma f\left(y_{n, N}\right)+\left(I-\alpha_{n} \mu V\right) G y_{n, N} ; \\
x_{n+1}=\beta_{n} x_{n}+\gamma_{n} y_{n}+\delta_{n} T y_{n},
\end{array}\right.
$$

for all $n \geq 0$, where

$V$ is a $\bar{\gamma}$-strongly positive bounded linear operator on $H$ and $f: C \rightarrow C$ is an

$l$-Lipschitz-continuous mapping with $0 \leq \gamma l<\mu \bar{\gamma}$;

$T: H \rightarrow H$ is a $\xi$-strict pseudocontraction and $S_{i}: C \rightarrow C$ is a nonexpansive mapping for each $i=1, \ldots, N$;

$R_{k}: C \rightarrow 2^{H}$ is a maximal monotone mapping and $B_{k}: C \rightarrow H$ is $\eta_{k}$-inverse-strongly monotone with $\left\{\lambda_{k, n}\right\} \subset\left[a_{k}, b_{k}\right] \subset\left(0,2 \eta_{k}\right)$ for each $k=1,2, \ldots, M$;

$F_{j}: C \rightarrow H$ is $\zeta_{j}$-inverse-strongly monotone and $G:=P_{C}\left(I-v_{1} F_{1}\right) P_{C}\left(I-v_{2} F_{2}\right)$ with

$v_{j} \in\left(0,2 \zeta_{j}\right)$ for $j=1,2$;

$\Theta, h: C \times C \rightarrow \mathbf{R}$ are two bifunctions satisfying the hypotheses of Lemma 2.9;

$\left\{\alpha_{n}\right\},\left\{\beta_{n}\right\}$ are sequences in $(0,1)$ with $0<\liminf _{n \rightarrow \infty} \beta_{n} \leq \limsup _{n \rightarrow \infty} \beta_{n}<1$;

$\left\{\gamma_{n}\right\},\left\{\delta_{n}\right\}$ are sequences in $[0,1]$ with $\beta_{n}+\gamma_{n}+\delta_{n}=1, \forall n \geq 0$;

$\left\{\beta_{n, i}\right\}_{i=1}^{N}$ are sequences in $(0,1)$ and $\left(\gamma_{n}+\delta_{n}\right) \xi \leq \gamma_{n}, \forall n \geq 0$;

$\left\{r_{n}\right\}$ is a sequence in $(0, \infty)$ with $\liminf _{n \rightarrow \infty} r_{n}>0$ and $\liminf _{n \rightarrow \infty} \delta_{n}>0$.

We start our main result from the following series of propositions.

Proposition 3.1 Let us suppose that $\Omega=\operatorname{Fix}(T) \cap \bigcap_{i=1}^{N} \operatorname{Fix}\left(S_{i}\right) \cap \bigcap_{k=1}^{M} \mathrm{I}\left(B_{k}, R_{k}\right) \cap \operatorname{GMEP}(\Theta$, h) $\cap \Xi \neq \emptyset$. Then the sequences $\left\{x_{n}\right\},\left\{y_{n}\right\},\left\{y_{n, i}\right\}$ for all $i,\left\{u_{n}\right\},\left\{v_{n}\right\}$ are bounded.

Proof Since $\lim _{n \rightarrow \infty} \alpha_{n}=0$ and $0<\liminf _{n \rightarrow \infty} \beta_{n} \leq \limsup _{n \rightarrow \infty} \beta_{n}<1$, we may assume, without loss of generality, that $\left\{\beta_{n}\right\} \subset[c, d] \subset(0,1)$ and $0<\alpha_{n} \mu \leq\|V\|^{-1}$ for all $n \geq 0$. Since $V$ is a $\bar{\gamma}$-strongly positive bounded linear operator on $H$, by Lemma 2.7 we know that

$$
\left\|I-\alpha_{n} \mu V\right\| \leq 1-\alpha_{n} \mu \bar{\gamma}, \quad \forall n \geq 0 .
$$

Put

$$
\Lambda_{n}^{k}=J_{R_{k}, \lambda_{k, n}}\left(I-\lambda_{k, n} B_{k}\right) J_{R_{k-1}, \lambda_{k-1, n}}\left(I-\lambda_{k-1, n} B_{k-1}\right) \cdots J_{R_{1}, \lambda_{1, n}}\left(I-\lambda_{1, n} B_{1}\right)
$$

for all $k \in\{1,2, \ldots, M\}$ and $n \geq 0$, and $\Lambda_{n}^{0}=I$, where $I$ is the identity mapping on $H$. Then we have that $v_{n}=\Lambda_{n}^{M} u_{n}$.

First of all, take a fixed $p \in \Omega$ arbitrarily. Utilizing (2.1) and Lemma 2.13 we have

$$
\begin{aligned}
\left\|v_{n}-p\right\| & =\left\|J_{R_{M}, \lambda_{M, n}}\left(I-\lambda_{M, n} A_{M}\right) \Lambda_{n}^{M-1} u_{n}-J_{R_{M}, \lambda_{M, n}}\left(I-\lambda_{M, n} A_{M}\right) \Lambda_{n}^{M-1} p\right\| \\
& \leq\left\|\left(I-\lambda_{M, n} A_{M}\right) \Lambda_{n}^{M-1} u_{n}-\left(I-\lambda_{M, n} A_{M}\right) \Lambda_{n}^{M-1} p\right\|
\end{aligned}
$$




$$
\begin{aligned}
& \leq\left\|\Lambda_{n}^{M-1} u_{n}-\Lambda_{n}^{M-1} p\right\| \\
& \ldots \\
& \leq\left\|\Lambda_{n}^{0} u_{n}-\Lambda_{n}^{0} p\right\| \\
& =\left\|u_{n}-p\right\| \leq\left\|x_{n}-p\right\| .
\end{aligned}
$$

Let us observe that, if $p \in \Omega$, then

$$
\left\|y_{n, 1}-p\right\| \leq\left\|v_{n}-p\right\| \leq\left\|u_{n}-p\right\| \leq\left\|x_{n}-p\right\|
$$

For all from $i=2$ to $i=N$, by induction, one proves that

$$
\left\|y_{n, i}-p\right\| \leq \beta_{n, i}\left\|v_{n}-p\right\|+\left(1-\beta_{n, i}\right)\left\|y_{n, i-1}-p\right\| \leq\left\|v_{n}-p\right\| \leq\left\|u_{n}-p\right\| \leq\left\|x_{n}-p\right\| .
$$

Thus we obtain, for every $i=1, \ldots, N$,

$$
\left\|y_{n, i}-p\right\| \leq\left\|v_{n}-p\right\| \leq\left\|u_{n}-p\right\| \leq\left\|x_{n}-p\right\| .
$$

For simplicity, we write $\tilde{p}=P_{C}\left(p-v_{2} F_{2} p\right), \tilde{y}_{n, N}=P_{C}\left(y_{n, N}-v_{2} F_{2} y_{n, N}\right)$, and $z_{n}=P_{C}\left(\tilde{y}_{n, N}-\right.$ $\left.\nu_{1} F_{1} \tilde{y}_{n, N}\right)$ for each $n \geq 1$. Then $z_{n}=G y_{n, N}$ and

$$
p=P_{C}\left(I-v_{1} F_{1}\right) \tilde{p}=P_{C}\left(I-v_{1} F_{1}\right) P_{C}\left(I-v_{2} F_{2}\right) p=G p .
$$

Since $F_{j}: C \rightarrow H$ is $\zeta_{j}$-inverse-strongly monotone and $0<v_{j}<2 \zeta_{j}$ for each $j=1,2$, we know that, for all $n \geq 0$,

$$
\begin{aligned}
&\left\|z_{n}-p\right\|^{2} \\
&=\left\|G y_{n, N}-p\right\|^{2} \\
&=\left\|P_{C}\left(I-v_{1} F_{1}\right) P_{C}\left(I-v_{2} F_{2}\right) y_{n, N}-P_{C}\left(I-v_{1} F_{1}\right) P_{C}\left(I-v_{2} F_{2}\right) p\right\|^{2} \\
& \leq\left\|\left(I-v_{1} F_{1}\right) P_{C}\left(I-v_{2} F_{2}\right) y_{n, N}-\left(I-v_{1} F_{1}\right) P_{C}\left(I-v_{2} F_{2}\right) p\right\|^{2} \\
&= \|\left[P_{C}\left(I-v_{2} F_{2}\right) y_{n, N}-P_{C}\left(I-v_{2} F_{2}\right) p\right] \\
&-v_{1}\left[F_{1} P_{C}\left(I-v_{2} F_{2}\right) y_{n, N}-F_{1} P_{C}\left(I-v_{2} F_{2}\right) p\right] \|^{2} \\
& \leq\left\|P_{C}\left(I-v_{2} F_{2}\right) y_{n, N}-P_{C}\left(I-v_{2} F_{2}\right) p\right\|^{2} \\
&+v_{1}\left(v_{1}-2 \zeta_{1}\right)\left\|F_{1} P_{C}\left(I-v_{2} F_{2}\right) y_{n, N}-F_{1} P_{C}\left(I-v_{2} F_{2}\right) p\right\|^{2} \\
& \leq\left\|P_{C}\left(I-v_{2} F_{2}\right) y_{n, N}-P_{C}\left(I-v_{2} F_{2}\right) p\right\|^{2} \\
& \leq\left\|\left(I-v_{2} F_{2}\right) y_{n, N}-\left(I-v_{2} F_{2}\right) p\right\|^{2} \\
&=\left\|\left(y_{n, N}-p\right)-v_{2}\left(F_{2} y_{n, N}-F_{2} p\right)\right\|^{2} \\
& \leq\left\|y_{n, N}-p\right\|^{2}+v_{2}\left(v_{2}-2 \zeta_{2}\right)\left\|F_{2} y_{n, N}-F_{2} p\right\|^{2} \\
& \leq\left\|y_{n, N}-p\right\|^{2} \leq\left\|v_{n}-p\right\|^{2} \leq\left\|u_{n}-p\right\|^{2} \leq\left\|x_{n}-p\right\|^{2} .
\end{aligned}
$$


Utilizing $G p=p$ and the nonexpansivity of $G$, from (3.1) and (3.4) we have

$$
\begin{aligned}
\| y_{n} & -p \|^{2} \\
& =\left\|\alpha_{n} \gamma\left(f\left(y_{n, N}\right)-f(p)\right)+\left(I-\alpha_{n} \mu V\right)\left(G y_{n, N}-p\right)+\alpha_{n}(\gamma f-\mu V) p\right\| \\
& \leq \alpha_{n} \gamma\left\|f\left(y_{n, N}\right)-f(p)\right\|+\left\|I-\alpha_{n} \mu V\right\|\left\|G y_{n, N}-p\right\|+\alpha_{n}\|(\gamma f-\mu V) p\| \\
& \leq \alpha_{n} \gamma l\left\|y_{n, N}-p\right\|+\left(1-\alpha_{n} \mu \bar{\gamma}\right)\left\|y_{n, N}-p\right\|+\alpha_{n}\|(\gamma f-\mu V) p\| \\
& =\left(1-\alpha_{n}(\mu \bar{\gamma}-\gamma l)\right)\left\|y_{n, N}-p\right\|+\alpha_{n}\|(\gamma f-\mu V) p\| \\
& =\left(1-\alpha_{n}(\mu \bar{\gamma}-\gamma l)\right)\left\|y_{n, N}-p\right\|+\alpha_{n}(\mu \bar{\gamma}-\gamma l) \frac{\|(\gamma f-\mu V) p\|}{\mu \bar{\gamma}-\gamma l} \\
& \leq \max \left\{\left\|y_{n, N}-p\right\|, \frac{\|(\gamma f-\mu V) p\|}{\mu \bar{\gamma}-\gamma l}\right\} \\
& \leq \max \left\{\left\|x_{n}-p\right\|, \frac{\|(\gamma f-\mu V) p\|}{\mu \bar{\gamma}-\gamma l}\right\} .
\end{aligned}
$$

Since $\left(\gamma_{n}+\delta_{n}\right) \xi \leq \gamma_{n}$ for all $n \geq 0$, utilizing Lemma 2.4 we obtain from (3.1) and (3.5)

$$
\begin{aligned}
\left\|x_{n+1}-p\right\|^{2} & =\left\|\beta_{n}\left(x_{n}-p\right)+\gamma_{n}\left(y_{n}-p\right)+\delta_{n}\left(T y_{n}-p\right)\right\| \\
& \leq \beta_{n}\left\|x_{n}-p\right\|+\left\|\gamma_{n}\left(y_{n}-p\right)+\delta_{n}\left(T y_{n}-p\right)\right\| \\
& \leq \beta_{n}\left\|x_{n}-p\right\|+\left(\gamma_{n}+\delta_{n}\right)\left\|y_{n}-p\right\| \\
& \leq \beta_{n}\left\|x_{n}-p\right\|+\left(\gamma_{n}+\delta_{n}\right) \max \left\{\left\|x_{n}-p\right\|, \frac{\|(\gamma f-\mu V) p\|}{\mu \bar{\gamma}-\gamma l}\right\} \\
& \leq \max \left\{\left\|x_{n}-p\right\|, \frac{\|(\gamma f-\mu V) p\|}{\mu \bar{\gamma}-\gamma l}\right\} .
\end{aligned}
$$

By induction, we get

$$
\left\|x_{n}-p\right\| \leq \max \left\{\left\|x_{0}-p\right\|, \frac{\|(\gamma f-\mu V) p\|}{\mu \bar{\gamma}-\gamma l}\right\}, \quad \forall n \geq 0 .
$$

This implies that $\left\{x_{n}\right\}$ is bounded and so are $\left\{F_{2} y_{n, N}\right\},\left\{F_{1} \tilde{y}_{n, N}\right\},\left\{\tilde{y}_{n, N}\right\},\left\{z_{n}\right\},\left\{u_{n}\right\},\left\{v_{n}\right\},\left\{y_{n}\right\}$, $\left\{y_{n, i}\right\}$ for each $i=1, \ldots, N$. Since $\left\|T y_{n}-p\right\| \leq \frac{1+\xi}{1-\xi}\left\|y_{n}-p\right\|,\left\{T y_{n}\right\}$ is also bounded.

Proposition 3.2 Let us suppose that $\Omega \neq \emptyset$. Moreover, let us suppose that the following hold:

(H0) $\lim _{n \rightarrow \infty} \alpha_{n}=0$ and $\sum_{n=0}^{\infty} \alpha_{n}=\infty$;

(H1) $\sum_{n=1}^{\infty}\left|\lambda_{k, n}-\lambda_{k, n-1}\right|<\infty$ or $\lim _{n \rightarrow \infty} \frac{\left|\lambda_{k, n}-\lambda_{k, n-1}\right|}{\alpha_{n}}=0$ for each $k=1, \ldots, M$;

(H2) $\sum_{n=1}^{\infty}\left|\alpha_{n}-\alpha_{n-1}\right|<\infty$ or $\lim _{n \rightarrow \infty} \frac{\left|\alpha_{n}-\alpha_{n-1}\right|}{\alpha_{n} \mid}=0$;

(H3) $\sum_{n=1}^{\infty}\left|\beta_{n, i}-\beta_{n-1, i}\right|<\infty$ or $\lim _{n \rightarrow \infty} \frac{\left|\beta_{n, i}-\beta_{n-1, i}\right|}{\alpha_{n}}=0$ for each $i=1, \ldots, N$;

(H4) $\sum_{n=1}^{\infty}\left|r_{n}-r_{n-1}\right|<\infty$ or $\lim _{n \rightarrow \infty} \frac{\left|r_{n}-r_{n-1}\right|}{\alpha_{n}}=0$;

(H5) $\sum_{n=1}^{\infty}\left|\beta_{n}-\beta_{n-1}\right|<\infty$ or $\lim _{n \rightarrow \infty} \frac{\left|\beta_{n}-\beta_{n-1}\right|}{\alpha_{n}}=0$;

(H6) $\sum_{n=1}^{\infty}\left|\frac{\gamma_{n}}{1-\beta_{n}}-\frac{\gamma_{n-1}}{1-\beta_{n-1}}\right|<\infty$ or $\lim _{n \rightarrow \infty} \frac{\alpha_{n}}{\alpha_{n}}\left|\frac{\gamma_{n}}{1-\beta_{n}}-\frac{\gamma_{n-1}}{1-\beta_{n-1}}\right|=0$.

Then $\lim _{n \rightarrow \infty}\left\|x_{n+1}-x_{n}\right\|=0$, i.e., $\left\{x_{n}\right\}$ is asymptotically regular.

Proof First, it is well known that $\left\{\beta_{n}\right\} \subset[c, d] \subset(0,1)$ as in the proof of Proposition 3.1. Taking into account $\liminf _{n \rightarrow \infty} r_{n}>0$, we may assume, without loss of generality, that 
$\left\{r_{n}\right\} \subset[\epsilon, \infty)$ for some $\epsilon>0$. First, we write $x_{n}=\beta_{n-1} x_{n-1}+\left(1-\beta_{n-1}\right) w_{n-1}, \forall n \geq 1$, where $w_{n-1}=\frac{x_{n}-\beta_{n-1} x_{n-1}}{1-\beta_{n-1}}$. It follows that for all $n \geq 1$

$$
\begin{aligned}
w_{n}-w_{n-1}= & \frac{x_{n+1}-\beta_{n} x_{n}}{1-\beta_{n}}-\frac{x_{n}-\beta_{n-1} x_{n-1}}{1-\beta_{n-1}} \\
= & \frac{\gamma_{n} y_{n}+\delta_{n} T y_{n}}{1-\beta_{n}}-\frac{\gamma_{n-1} y_{n-1}+\delta_{n-1} T y_{n-1}}{1-\beta_{n-1}} \\
= & \frac{\gamma_{n}\left(y_{n}-y_{n-1}\right)+\delta_{n}\left(T y_{n}-T y_{n-1}\right)}{1-\beta_{n}}+\left(\frac{\gamma_{n}}{1-\beta_{n}}-\frac{\gamma_{n-1}}{1-\beta_{n-1}}\right) y_{n-1} \\
& +\left(\frac{\delta_{n}}{1-\beta_{n}}-\frac{\delta_{n-1}}{1-\beta_{n-1}}\right) T y_{n-1} .
\end{aligned}
$$

Since $\left(\gamma_{n}+\delta_{n}\right) \xi \leq \gamma_{n}$ for all $n \geq 0$, utilizing Lemma 2.4 we have

$$
\left\|\gamma_{n}\left(y_{n}-y_{n-1}\right)+\delta_{n}\left(T y_{n}-T y_{n-1}\right)\right\| \leq\left(\gamma_{n}+\delta_{n}\right)\left\|y_{n}-y_{n-1}\right\| .
$$

Next, we estimate $\left\|y_{n}-y_{n-1}\right\|$. Observe that

$$
\begin{aligned}
\| z_{n}- & z_{n-1} \|^{2} \\
= & \left\|P_{C}\left(I-v_{1} F_{1}\right) P_{C}\left(I-v_{2} F_{2}\right) y_{n, N}-P_{C}\left(I-v_{1} F_{1}\right) P_{C}\left(I-v_{2} F_{2}\right) y_{n-1, N}\right\|^{2} \\
\leq & \left\|\left(I-v_{1} F_{1}\right) P_{C}\left(I-v_{2} F_{2}\right) y_{n, N}-\left(I-v_{1} F_{1}\right) P_{C}\left(I-v_{2} F_{2}\right) y_{n-1, N}\right\|^{2} \\
= & \|\left[P_{C}\left(I-v_{2} F_{2}\right) y_{n, N}-P_{C}\left(I-v_{2} F_{2}\right) y_{n-1, N}\right] \\
& -v_{1}\left[F_{1} P_{C}\left(I-v_{2} F_{2}\right) y_{n, N}-F_{1} P_{C}\left(I-v_{2} F_{2}\right) y_{n-1, N}\right] \|^{2} \\
\leq & \left\|P_{C}\left(I-v_{2} F_{2}\right) y_{n, N}-P_{C}\left(I-v_{2} F_{2}\right) y_{n-1, N}\right\|^{2} \\
& -v_{1}\left(2 \zeta_{1}-v_{1}\right)\left\|F_{1} P_{C}\left(I-v_{2} F_{2}\right) y_{n, N}-F_{1} P_{C}\left(I-v_{2} F_{2}\right) y_{n-1, N}\right\|^{2} \\
\leq & \left\|P_{C}\left(I-v_{2} F_{2}\right) y_{n, N}-P_{C}\left(I-v_{2} F_{2}\right) y_{n-1, N}\right\|^{2} \\
\leq & \left\|\left(I-v_{2} F_{2}\right) y_{n, N}-\left(I-v_{2} F_{2}\right) y_{n-1, N}\right\|^{2} \\
= & \left\|\left(y_{n, N}-y_{n-1, N}\right)-v_{2}\left(F_{2} y_{n, N}-F_{2} y_{n-1, N}\right)\right\|^{2} \\
\leq & \left\|y_{n, N}-y_{n-1, N}\right\|^{2}-v_{2}\left(2 \zeta_{2}-v_{2}\right)\left\|F_{2} y_{n, N}-F_{2} y_{n-1, N}\right\|^{2} \\
\leq & \left\|y_{n, N}-y_{n-1, N}\right\|^{2} .
\end{aligned}
$$

Also, from (3.1) we have

$$
\left\{\begin{array}{l}
y_{n}=\alpha_{n} \gamma f\left(y_{n, N}\right)+\left(I-\alpha_{n} \mu V\right) z_{n}, \\
y_{n-1}=\alpha_{n-1} \gamma f\left(y_{n-1, N}\right)+\left(I-\alpha_{n-1} \mu V\right) z_{n-1}, \quad \forall n \geq 1 .
\end{array}\right.
$$

Simple calculations show that

$$
\begin{aligned}
y_{n}-y_{n-1}= & \left(I-\alpha_{n} \mu V\right)\left(z_{n}-z_{n-1}\right)+\left(\alpha_{n}-\alpha_{n-1}\right)\left(\gamma f\left(y_{n-1, N}\right)-\mu V z_{n-1}\right) \\
& +\alpha_{n} \gamma\left(f\left(y_{n, N}\right)-f\left(y_{n-1, N}\right)\right) .
\end{aligned}
$$


Then passing to the norm we get from (3.9)

$$
\begin{aligned}
\left\|y_{n}-y_{n-1}\right\| \leq & \left\|I-\alpha_{n} \mu V\right\|\left\|z_{n}-z_{n-1}\right\|+\left|\alpha_{n}-\alpha_{n-1}\right|\left\|\gamma f\left(y_{n-1, N}\right)-\mu V z_{n-1}\right\| \\
& +\alpha_{n} \gamma\left\|f\left(y_{n, N}\right)-f\left(y_{n-1, N}\right)\right\| \\
\leq & \left(1-\alpha_{n} \mu \bar{\gamma}\right)\left\|y_{n, N}-y_{n-1, N}\right\|+\tilde{M}\left|\alpha_{n}-\alpha_{n-1}\right|+\alpha_{n} \gamma l\left\|y_{n, N}-y_{n-1, N}\right\| \\
= & \left(1-\alpha_{n}(\mu \bar{\gamma}-\gamma l)\right)\left\|y_{n, N}-y_{n-1, N}\right\|+\tilde{M}\left|\alpha_{n}-\alpha_{n-1}\right|,
\end{aligned}
$$

where $\sup _{n \geq 0}\left\|\gamma f\left(y_{n, N}\right)-\mu V z_{n}\right\| \leq \tilde{M}$ for some $\tilde{M}>0$. In the meantime, by the definition of $y_{n, i}$ one obtains, for all $i=N, \ldots, 2$,

$$
\begin{aligned}
\left\|y_{n, i}-y_{n-1, i}\right\| \leq & \beta_{n, i}\left\|v_{n}-v_{n-1}\right\|+\left\|S_{i} v_{n-1}-y_{n-1, i-1}\right\|\left|\beta_{n, i}-\beta_{n-1, i}\right| \\
& +\left(1-\beta_{n, i}\right)\left\|y_{n, i-1}-y_{n-1, i-1}\right\| .
\end{aligned}
$$

In the case $i=1$, we have

$$
\begin{aligned}
\left\|y_{n, 1}-y_{n-1,1}\right\| & \leq \beta_{n, 1}\left\|v_{n}-v_{n-1}\right\|+\left\|S_{1} v_{n-1}-v_{n-1}\right\|\left|\beta_{n, 1}-\beta_{n-1,1}\right|+\left(1-\beta_{n, 1}\right)\left\|v_{n}-v_{n-1}\right\| \\
& =\left\|v_{n}-v_{n-1}\right\|+\left\|S_{1} v_{n-1}-v_{n-1}\right\|\left|\beta_{n, 1}-\beta_{n-1,1}\right| .
\end{aligned}
$$

Substituting (3.12) in all (3.11)-type expressions one obtains for $i=2, \ldots, N$

$$
\begin{aligned}
\left\|y_{n, i}-y_{n-1, i}\right\| \leq & \left\|v_{n}-v_{n-1}\right\|+\sum_{k=2}^{i}\left\|S_{k} v_{n-1}-y_{n-1, k-1}\right\|\left|\beta_{n, k}-\beta_{n-1, k}\right| \\
& +\left\|S_{1} v_{n-1}-v_{n-1}\right\|\left|\beta_{n, 1}-\beta_{n-1,1}\right| .
\end{aligned}
$$

This together with (3.10) implies that

$$
\begin{aligned}
& \left\|y_{n}-y_{n-1}\right\| \\
& \leq\left(1-\alpha_{n}(\mu \bar{\gamma}-\gamma l)\right)\left\|y_{n, N}-y_{n-1, N}\right\|+\tilde{M}\left|\alpha_{n}-\alpha_{n-1}\right| \\
& \leq\left(1-\alpha_{n}(\mu \bar{\gamma}-\gamma l)\right)\left[\left\|v_{n}-v_{n-1}\right\|+\sum_{k=2}^{N}\left\|S_{k} v_{n-1}-y_{n-1, k-1}\right\|\left|\beta_{n, k}-\beta_{n-1, k}\right|\right. \\
& \left.\quad+\left\|S_{1} v_{n-1}-v_{n-1}\right\|\left|\beta_{n, 1}-\beta_{n-1,1}\right|\right]+\tilde{M}\left|\alpha_{n}-\alpha_{n-1}\right| \\
& \quad\left(1-\alpha_{n}(\mu \bar{\gamma}-\gamma l)\right)\left\|v_{n}-v_{n-1}\right\|+\sum_{k=2}^{N}|| S_{k} v_{n-1}-y_{n-1, k-1} \|\left|\beta_{n, k}-\beta_{n-1, k}\right| \\
& \quad+\left\|S_{1} v_{n-1}-v_{n-1}\right\|\left|\beta_{n, 1}-\beta_{n-1,1}\right|+\tilde{M}\left|\alpha_{n}-\alpha_{n-1}\right| .
\end{aligned}
$$

Furthermore, utilizing (2.1) and (2.2), we obtain

$$
\begin{aligned}
& \left\|v_{n}-v_{n-1}\right\| \\
& \quad=\left\|\Lambda_{n}^{M} u_{n}-\Lambda_{n-1}^{M} u_{n-1}\right\|
\end{aligned}
$$




$$
\begin{aligned}
= & \left\|J_{R_{M}, \lambda_{M, n}}\left(I-\lambda_{M, n} B_{M}\right) \Lambda_{n}^{M-1} u_{n}-J_{R_{M}, \lambda_{M, n-1}}\left(I-\lambda_{M, n-1} B_{M}\right) \Lambda_{n-1}^{M-1} u_{n-1}\right\| \\
\leq & \left\|J_{R_{M}, \lambda_{M, n}}\left(I-\lambda_{M, n} B_{M}\right) \Lambda_{n}^{M-1} u_{n}-J_{R_{M}, \lambda_{M, n}}\left(I-\lambda_{M, n-1} B_{M}\right) \Lambda_{n}^{M-1} u_{n}\right\| \\
& +\left\|J_{R_{M}, \lambda, \lambda_{M, n}}\left(I-\lambda_{M, n-1} B_{M}\right) \Lambda_{n}^{M-1} u_{n}-J_{R_{M}, \lambda_{M, n-1}}\left(I-\lambda_{M, n-1} B_{M}\right) \Lambda_{n-1}^{M-1} u_{n-1}\right\| \\
\leq & \left\|\left(I-\lambda_{M, n} B_{M}\right) \Lambda_{n}^{M-1} u_{n}-\left(I-\lambda_{M, n-1} B_{M}\right) \Lambda_{n}^{M-1} u_{n}\right\| \\
& +\left\|\left(I-\lambda_{M, n-1} B_{M}\right) \Lambda_{n}^{M-1} u_{n}-\left(I-\lambda_{M, n-1} B_{M}\right) \Lambda_{n-1}^{M-1} u_{n-1}\right\|+\left|\lambda_{M, n}-\lambda_{M, n-1}\right| \\
& \times\left(\frac{1}{\lambda_{M, n}}\left\|J_{R_{M}, \lambda_{M, n}}\left(I-\lambda_{M, n-1} B_{M}\right) \Lambda_{n}^{M-1} u_{n}-\left(I-\lambda_{M, n-1} B_{M}\right) \Lambda_{n-1}^{M-1} u_{n-1}\right\|\right. \\
& \left.+\frac{1}{\lambda_{M, n-1}}\left\|\left(I-\lambda_{M, n-1} B_{M}\right) \Lambda_{n}^{M-1} u_{n}-J_{R_{M}, \lambda_{M, n-1}}\left(I-\lambda_{M, n-1} B_{M}\right) \Lambda_{n-1}^{M-1} u_{n-1}\right\|\right) \\
\leq & \left|\lambda_{M, n}-\lambda_{M, n-1}\right|\left(\left\|B_{M} \Lambda_{n}^{M-1} u_{n}\right\|+\widehat{M}\right)+\left\|\Lambda_{n}^{M-1} u_{n}-\Lambda_{n-1}^{M-1} u_{n-1}\right\| \\
\leq & \left|\lambda_{M, n}-\lambda_{M, n-1}\right|\left(\left\|B_{M} \Lambda_{n}^{M-1} u_{n}\right\|+\widehat{M}\right) \\
& +\left|\lambda_{M-1, n}-\lambda_{M-1, n-1}\right|\left(\left\|B_{M-1} \Lambda_{n}^{M-2} u_{n}\right\|+\widehat{M}\right)+\left\|\Lambda_{n}^{M-2} u_{n}-\Lambda_{n-1}^{M-2} u_{n-1}\right\| \\
& \ldots \\
\leq & \left|\lambda_{M, n}-\lambda_{M, n-1}\right|\left(\left\|B_{M} \Lambda_{n}^{M-1} u_{n}\right\|+\widehat{M}\right) \\
& +\left|\lambda_{M-1, n}-\lambda_{M-1, n-1}\right|\left(\left\|B_{M-1} \Lambda_{n}^{M-2} u_{n}\right\|+\widehat{M}\right)+\cdots \\
& +\left|\lambda_{1, n}-\lambda_{1, n-1}\right|\left(\left\|B_{1} \Lambda_{n}^{0} u_{n}\right\|+\widehat{M}\right)+\left\|\Lambda_{n}^{0} u_{n}-\Lambda_{n-1}^{0} u_{n-1}\right\| \\
\leq & \widetilde{M}_{0} \sum_{k=1}^{M}\left|\lambda_{k, n}-\lambda_{k, n-1}\right|+\left\|u_{n}-u_{n-1}\right\|, \\
&
\end{aligned}
$$

where

$$
\begin{aligned}
& \sup _{n \geq 1,1 \leq k \leq M}\left\{\frac{1}{\lambda_{k, n}}\left\|J_{R_{k}, \lambda_{k, n}}\left(I-\lambda_{k, n-1} B_{k}\right) \Lambda_{n}^{k-1} u_{n}-\left(I-\lambda_{k, n-1} B_{k}\right) \Lambda_{n-1}^{k-1} u_{n-1}\right\|\right. \\
& \left.\quad+\frac{1}{\lambda_{k, n-1}}\left\|\left(I-\lambda_{k, n-1} B_{k}\right) \Lambda_{n}^{k-1} u_{n}-J_{R_{k}, \lambda_{k, n-1}}\left(I-\lambda_{k, n-1} B_{k}\right) \Lambda_{n-1}^{k-1} u_{n-1}\right\|\right\} \leq \widehat{M},
\end{aligned}
$$

for some $\widehat{M}>0$ and $\sup _{n \geq 0}\left\{\sum_{k=1}^{M}\left\|B_{k} \Lambda_{n}^{k-1} u_{n}\right\|+\widehat{M}\right\} \leq \widetilde{M}_{0}$ for some $\widetilde{M}_{0}>0$.

By Lemma 2.10, we know that

$$
\left\|u_{n}-u_{n-1}\right\| \leq\left\|x_{n}-x_{n-1}\right\|+L\left|1-\frac{r_{n-1}}{r_{n}}\right|
$$

where $L=\sup _{n \geq 0}\left\|u_{n}-x_{n}\right\|$. So, combining (3.13)-(3.15), we obtain

$$
\begin{aligned}
& \left\|y_{n}-y_{n-1}\right\| \\
& \leq \quad\left(1-\alpha_{n}(\mu \bar{\gamma}-\gamma l)\right)\left\|v_{n}-v_{n-1}\right\|+\sum_{k=2}^{N}\left\|S_{k} v_{n-1}-y_{n-1, k-1}\right\|\left|\beta_{n, k}-\beta_{n-1, k}\right| \\
& \quad+\left\|S_{1} v_{n-1}-v_{n-1}\right\|\left|\beta_{n, 1}-\beta_{n-1,1}\right|+\tilde{M}\left|\alpha_{n}-\alpha_{n-1}\right| \\
& \leq\left(1-\alpha_{n}(\mu \bar{\gamma}-\gamma l)\right)\left[\tilde{M}_{0} \sum_{k=1}^{M}\left|\lambda_{k, n}-\lambda_{k, n-1}\right|+\left\|u_{n}-u_{n-1}\right\|\right]
\end{aligned}
$$




$$
\begin{aligned}
& +\sum_{k=2}^{N}\left\|S_{k} v_{n-1}-y_{n-1, k-1}\right\|\left|\beta_{n, k}-\beta_{n-1, k}\right| \\
& +|| S_{1} v_{n-1}-v_{n-1} \|\left|\beta_{n, 1}-\beta_{n-1,1}\right|+\widetilde{M}\left|\alpha_{n}-\alpha_{n-1}\right| \\
\leq & \left(1-\alpha_{n}(\mu \bar{\gamma}-\gamma l)\right)\left[\tilde{M}_{0} \sum_{k=1}^{M}\left|\lambda_{k, n}-\lambda_{k, n-1}\right|+|| x_{n}-x_{n-1}|+L| 1-\frac{r_{n-1}}{r_{n}} \mid\right] \\
& +\sum_{k=2}^{N}\left\|S_{k} v_{n-1}-y_{n-1, k-1}\right\|\left|\beta_{n, k}-\beta_{n-1, k}\right| \\
& +\left\|S_{1} v_{n-1}-v_{n-1}\right\|\left|\beta_{n, 1}-\beta_{n-1,1}\right|+\widetilde{M}\left|\alpha_{n}-\alpha_{n-1}\right| \\
\leq & \left(1-\alpha_{n}(\mu \bar{\gamma}-\gamma l)\right)\left\|x_{n}-x_{n-1}\right\|+\tilde{M}_{0} \sum_{k=1}^{M}\left|\lambda_{k, n}-\lambda_{k, n-1}\right|+L\left|1-\frac{r_{n-1}}{r_{n}}\right| \\
& +\sum_{k=2}^{N}\left\|S_{k} v_{n-1}-y_{n-1, k-1}\right\|\left|\beta_{n, k}-\beta_{n-1, k}\right| \\
& +\left\|S_{1} v_{n-1}-v_{n-1}\right\|\left|\beta_{n, 1}-\beta_{n-1,1}\right|+\widetilde{M}\left|\alpha_{n}-\alpha_{n-1}\right| \\
\leq & \left(1-\alpha_{n}(\mu \bar{\gamma}-\gamma l)\right)\left\|x_{n}-x_{n-1}\right\|+\widetilde{M}_{1}\left[\sum_{k=1}^{M}\left|\lambda_{k, n}-\lambda_{k, n-1}\right|+\frac{\left|r_{n}-r_{n-1}\right|}{r_{n}}\right. \\
& \left.+\sum_{k=1}^{N}\left|\beta_{n, k}-\beta_{n-1, k}\right|+\left|\alpha_{n}-\alpha_{n-1}\right|\right], \\
& \left.+\sum_{k=2}^{N}\left|\beta_{n, k}-\beta_{n-1, k}\right|+\left|\beta_{n, 1}-\beta_{n-1,1}\right|+\left|\alpha_{n}-\alpha_{n-1}\right|\right] \\
\leq & \left(1-\alpha_{n}(\mu \bar{\gamma}-\gamma l)\right)\left\|x_{n}-x_{n-1}\right\|+\tilde{M}_{1}\left[\frac{\left|r_{n}-r_{n-1}\right|}{\epsilon}+\sum_{k=1}^{M}\left|\lambda_{k, n}-\lambda_{k, n-1}\right|\right. \\
&
\end{aligned}
$$

where $\sup _{n \geq 1}\left\{\widetilde{M}_{0}+L+\sum_{k=2}^{N}\left\|S_{k} v_{n-1}-y_{n-1, k-1}\right\|+\left\|S_{1} v_{n-1}-v_{n-1}\right\|+\widetilde{M}\right\} \leq \widetilde{M}_{1}$ for some $\widetilde{M}_{1}>0$.

This together with (3.7)-(3.8) implies that

$$
\begin{aligned}
\| w_{n} & -w_{n-1} \| \\
\leq & \frac{\left\|\gamma_{n}\left(y_{n}-y_{n-1}\right)+\delta_{n}\left(T y_{n}-T y_{n-1}\right)\right\|}{1-\beta_{n}}+\left|\frac{\gamma_{n}}{1-\beta_{n}}-\frac{\gamma_{n-1}}{1-\beta_{n-1}}\right|\left\|y_{n-1}\right\| \\
& +\left|\frac{\delta_{n}}{1-\beta_{n}}-\frac{\delta_{n-1}}{1-\beta_{n-1}}\right|\left\|T y_{n-1}\right\| \\
\leq & \frac{\left(\gamma_{n}+\delta_{n}\right)\left\|y_{n}-y_{n-1}\right\|}{1-\beta_{n}}+\left|\frac{\gamma_{n}}{1-\beta_{n}}-\frac{\gamma_{n-1}}{1-\beta_{n-1}}\right|\left\|y_{n-1}\right\|+\left|\frac{\delta_{n}}{1-\beta_{n}}-\frac{\delta_{n-1}}{1-\beta_{n-1}}\right|\left\|T y_{n-1}\right\| \\
= & \left\|y_{n}-y_{n-1}\right\|+\left|\frac{\gamma_{n}}{1-\beta_{n}}-\frac{\gamma_{n-1}}{1-\beta_{n-1}}\right|\left(\left\|y_{n-1}\right\|+\left\|T y_{n-1}\right\|\right) \\
\leq & \left(1-\alpha_{n}(\mu \bar{\gamma}-\gamma l)\right)\left\|x_{n}-x_{n-1}\right\|+\tilde{M}_{1}\left[\frac{\left|r_{n}-r_{n-1}\right|}{\epsilon}+\sum_{k=1}^{M}\left|\lambda_{k, n}-\lambda_{k, n-1}\right|\right. \\
& \left.+\sum_{k=1}^{N}\left|\beta_{n, k}-\beta_{n-1, k}\right|+\left|\alpha_{n}-\alpha_{n-1}\right|\right]+\left|\frac{\gamma_{n}}{1-\beta_{n}}-\frac{\gamma_{n-1}}{1-\beta_{n-1}}\right|\left(\left\|y_{n-1}\right\|+\left\|T y_{n-1}\right\|\right)
\end{aligned}
$$




$$
\begin{aligned}
\leq & \left(1-\alpha_{n}(\mu \bar{\gamma}-\gamma l)\right)\left\|x_{n}-x_{n-1}\right\|+\tilde{M}_{2}\left[\frac{\left|r_{n}-r_{n-1}\right|}{\epsilon}+\sum_{k=1}^{M}\left|\lambda_{k, n}-\lambda_{k, n-1}\right|\right. \\
& \left.+\sum_{k=1}^{N}\left|\beta_{n, k}-\beta_{n-1, k}\right|+\left|\alpha_{n}-\alpha_{n-1}\right|+\left|\frac{\gamma_{n}}{1-\beta_{n}}-\frac{\gamma_{n-1}}{1-\beta_{n-1}}\right|\right]
\end{aligned}
$$

where $\sup _{n \geq 0}\left\{\widetilde{M}_{1}+\left\|y_{n}\right\|+\left\|T y_{n}\right\|\right\} \leq \widetilde{M}_{2}$ for some $\tilde{M}_{2}>0$.

Further, we observe that

$$
\left\{\begin{array}{l}
x_{n+1}=\beta_{n} x_{n}+\left(1-\beta_{n}\right) w_{n} \\
x_{n}=\beta_{n-1} x_{n-1}+\left(1-\beta_{n-1}\right) w_{n-1}, \quad \forall n \geq 1
\end{array}\right.
$$

Simple calculations show that

$$
x_{n+1}-x_{n}=\left(1-\beta_{n}\right)\left(w_{n}-w_{n-1}\right)+\left(\beta_{n}-\beta_{n-1}\right)\left(x_{n-1}-w_{n-1}\right)+\beta_{n}\left(x_{n}-x_{n-1}\right) .
$$

Then passing to the norm we get from (3.16)

$$
\begin{aligned}
\| x_{n+1} & -x_{n} \| \\
\leq & \left(1-\beta_{n}\right)\left\|w_{n}-w_{n-1}\right\|+\left|\beta_{n}-\beta_{n-1}\right|\left\|x_{n-1}-w_{n-1}\right\|+\beta_{n}\left\|x_{n}-x_{n-1}\right\| \\
\leq & \left(1-\beta_{n}\right)\left\{\left(1-\alpha_{n}(\mu \bar{\gamma}-\gamma l)\right) \| x_{n}-x_{n-1} \mid+\tilde{M}_{2}\left[\frac{\left|r_{n}-r_{n-1}\right|}{\epsilon}+\sum_{k=1}^{M}\left|\lambda_{k, n}-\lambda_{k, n-1}\right|\right.\right. \\
& \left.\left.+\sum_{k=1}^{N}\left|\beta_{n, k}-\beta_{n-1, k}\right|+\left|\alpha_{n}-\alpha_{n-1}\right|+\left|\frac{\gamma_{n}}{1-\beta_{n}}-\frac{\gamma_{n-1}}{1-\beta_{n-1}}\right|\right]\right\} \\
& +\left|\beta_{n}-\beta_{n-1}\right| \mid x_{n-1}-w_{n-1}\left\|+\beta_{n}\right\| x_{n}-x_{n-1} \| \\
\leq & \left(1-(\mu \bar{\gamma}-\gamma l)\left(1-\beta_{n}\right) \alpha_{n}\right)\left\|x_{n}-x_{n-1}\right\|+\tilde{M}_{2}\left[\frac{\left|r_{n}-r_{n-1}\right|}{\epsilon}+\sum_{k=1}^{M}\left|\lambda_{k, n}-\lambda_{k, n-1}\right|\right. \\
& \left.+\sum_{k=1}^{N}\left|\beta_{n, k}-\beta_{n-1, k}\right|+\left|\alpha_{n}-\alpha_{n-1}\right|+\left|\frac{\gamma_{n}}{1-\beta_{n}}-\frac{\gamma_{n-1}}{1-\beta_{n-1}}\right|\right]+\left|\beta_{n}-\beta_{n-1}\right|\left|x_{n-1}-w_{n-1}\right| \mid \\
\leq & \left(1-(\mu \bar{\gamma}-\gamma l)(1-d) \alpha_{n}\right)\left\|x_{n}-x_{n-1}\right\|+\tilde{M}_{3}\left[\frac{\left|r_{n}-r_{n-1}\right|}{\epsilon}+\sum_{k=1}^{M}\left|\lambda_{k, n}-\lambda_{k, n-1}\right|\right. \\
& \left.+\sum_{k=1}^{N}\left|\beta_{n, k}-\beta_{n-1, k}\right|+\left|\alpha_{n}-\alpha_{n-1}\right|+\left|\frac{\gamma_{n}}{1-\beta_{n}}-\frac{\gamma_{n-1}}{1-\beta_{n-1}}\right|+\left|\beta_{n}-\beta_{n-1}\right|\right], \\
& N(3.17)
\end{aligned}
$$

where $\sup _{n \geq 0}\left\{\tilde{M}_{2}+\left\|x_{n}-w_{n}\right\|\right\} \leq \tilde{M}_{3}$ for some $\tilde{M}_{3}>0$. By hypotheses (H0)-(H6) and Lemma 2.8, we obtain the claim.

Proposition 3.3 Let us suppose that $\Omega \neq \emptyset$. Let us suppose that $\left\{x_{n}\right\}$ is asymptotically regular. Then $\left\|x_{n}-u_{n}\right\|=\left\|x_{n}-T_{r_{n}} x_{n}\right\| \rightarrow 0$ and $\left\|x_{n}-v_{n}\right\| \rightarrow 0$ as $n \rightarrow \infty$. 
Proof Take a fixed $p \in \Omega$ arbitrarily. We recall that, by the firm nonexpansivity of $T_{r_{n}}$, a standard calculation (see [44]) shows that for $p \in \operatorname{GMEP}(\Theta, h)$

$$
\left\|u_{n}-p\right\|^{2} \leq\left\|x_{n}-p\right\|^{2}-\left\|x_{n}-u_{n}\right\|^{2} .
$$

Observe that

$$
\begin{aligned}
\left\|\Lambda_{n}^{k} u_{n}-p\right\|^{2} & =\left\|J_{R_{k}, \lambda_{k, n}}\left(I-\lambda_{k, n} B_{k}\right) \Lambda_{n}^{k-1} u_{n}-J_{R_{k}, \lambda_{k, n}}\left(I-\lambda_{k, n} B_{k}\right) p\right\|^{2} \\
& \leq\left\|\left(I-\lambda_{k, n} B_{k}\right) \Lambda_{n}^{k-1} u_{n}-\left(I-\lambda_{k, n} B_{k}\right) p\right\|^{2} \\
& \leq\left\|\Lambda_{n}^{k-1} u_{n}-p\right\|^{2}+\lambda_{k, n}\left(\lambda_{k, n}-2 \eta_{k}\right)\left\|B_{k} \Lambda_{n}^{k-1} u_{n}-B_{k} p\right\|^{2} \\
& \leq\left\|u_{n}-p\right\|^{2}+\lambda_{k, n}\left(\lambda_{k, n}-2 \eta_{k}\right)\left\|B_{k} \Lambda_{n}^{k-1} u_{n}-B_{k} p\right\|^{2} \\
& \leq\left\|x_{n}-p\right\|^{2}+\lambda_{k, n}\left(\lambda_{k, n}-2 \eta_{k}\right)\left\|B_{k} \Lambda_{n}^{k-1} u_{n}-B_{k} p\right\|^{2},
\end{aligned}
$$

for each $k \in\{1,2, \ldots, M\}$.

Utilizing Lemmas 2.1 and 2.2(b), we obtain from $0 \leq \gamma l<\mu \bar{\gamma}$, (3.1), (3.4), (3.18), and (3.19) that

$$
\begin{aligned}
\| y_{n}- & p \|^{2} \\
= & \left\|\alpha_{n} \gamma\left(f\left(y_{n, N}\right)-f(p)\right)+\left(I-\alpha_{n} \mu V\right)\left(z_{n}-p\right)+\alpha_{n}(\gamma f-\mu V) p\right\|^{2} \\
\leq & \left\|\alpha_{n} \gamma\left(f\left(y_{n, N}\right)-f(p)\right)+\left(I-\alpha_{n} \mu V\right)\left(z_{n}-p\right)\right\|^{2}+2 \alpha_{n}\left|(\gamma f-\mu V) p, y_{n}-p\right\rangle \\
\leq & {\left[\alpha_{n} \gamma\left\|f\left(y_{n, N}\right)-f(p)\right\|+\left\|I-\alpha_{n} \mu V\right\|\left\|z_{n}-p\right\|\right]^{2}+2 \alpha_{n}\left\langle(\gamma f-\mu V) p, y_{n}-p\right\rangle } \\
\leq & {\left[\alpha_{n} \gamma l\left\|y_{n, N}-p\right\|+\left(1-\alpha_{n} \mu \bar{\gamma}\right)\left\|z_{n}-p\right\|\right]^{2}+2 \alpha_{n}\left\langle(\gamma f-\mu V) p, y_{n}-p\right\rangle } \\
= & {\left[\alpha_{n} \mu \bar{\gamma} \frac{\gamma l}{\mu \bar{\gamma}}\left\|y_{n, N}-p\right\|+\left(1-\alpha_{n} \mu \bar{\gamma}\right)\left\|z_{n}-p\right\|^{2}+2 \alpha_{n}\left((\gamma f-\mu V) p, y_{n}-p\right\rangle\right.} \\
\leq & \alpha_{n} \mu \bar{\gamma} \frac{(\gamma l)^{2}}{(\mu \bar{\gamma})^{2}}\left\|y_{n, N}-p\right\|^{2}+\left(1-\alpha_{n} \mu \bar{\gamma}\right)\left\|z_{n}-p\right\|^{2}+2 \alpha_{n}\left((\gamma f-\mu V) p, y_{n}-p\right\rangle \\
\leq & \alpha_{n} \mu \bar{\gamma}\left\|y_{n, N}-p\right\|^{2}+\left\|z_{n}-p\right\|^{2}+2 \alpha_{n}\|(\gamma f-\mu V) p\|\left\|y_{n}-p\right\| \\
\leq & \alpha_{n} \mu \bar{\gamma}\left\|y_{n, N}-p\right\|^{2}+\left\|y_{n, N}-p\right\|^{2}-v_{2}\left(2 \zeta_{2}-v_{2}\right)\left\|F_{2} y_{n, N}-F_{2} p\right\|^{2} \\
& -v_{1}\left(2 \zeta_{1}-v_{1}\right)\left\|F_{1} \tilde{y}_{n, N}-F_{1} \tilde{p}\right\|^{2}+2 \alpha_{n}\|(\gamma f-\mu V) p\|\left\|y_{n}-p\right\| \\
\leq & \alpha_{n} \mu \bar{\gamma}\left\|y_{n, N}-p\right\|^{2}+\left\|v_{n}-p\right\|^{2}-v_{2}\left(2 \zeta_{2}-v_{2}\right)\left\|F_{2} y_{n, N}-F_{2} p\right\|^{2} \\
& -v_{1}\left(2 \zeta_{1}-v_{1}\right)\left\|F_{1} \tilde{y}_{n, N}-F_{1} \tilde{p}\right\|^{2}+2 \alpha_{n}\|(\gamma f-\mu V) p\|\left\|y_{n}-p\right\| \\
\leq & \alpha_{n} \mu \bar{\gamma}\left\|y_{n, N}-p\right\|^{2}+\left\|\Lambda_{n}^{k} u_{n}-p\right\|^{2}-v_{2}\left(2 \zeta_{2}-v_{2}\right)\left\|F_{2} y_{n, N}-F_{2} p\right\|^{2} \\
& -v_{1}\left(2 \zeta_{1}-v_{1}\right)\left\|F_{1} \tilde{y}_{n, N}-F_{1} \tilde{p}\right\|^{2}+2 \alpha_{n}\|(\gamma f-\mu V) p\|\left\|y_{n}-p\right\| \\
\leq & \alpha_{n} \mu \bar{\gamma}\left\|y_{n, N}-p\right\|^{2}+\left\|u_{n}-p\right\|^{2}+\lambda_{k, n}\left(\lambda_{k, n}-2 \eta_{k}\right)\left\|B_{k} \Lambda_{n}^{k-1} u_{n}-B_{k} p\right\|^{2} \\
& -v_{2}\left(2 \zeta_{2}-v_{2}\right)\left\|F_{2} y_{n, N}-F_{2} p\right\|^{2}-v_{1}\left(2 \zeta_{1}-v_{1}\right)\left\|F_{1} \tilde{y}_{n, N}-F_{1} \tilde{p}\right\|^{2} \\
& +2 \alpha_{n}\|(\gamma f-\mu V) p\|\left\|y_{n}-p\right\| \\
\leq & \alpha_{n} \mu \bar{\gamma}\left\|y_{n, N}-p\right\|^{2}+\left\|x_{n}-p\right\|^{2}-\left\|x_{n}-u_{n}\right\|^{2}+\lambda_{k, n}\left(\lambda_{k, n}-2 \eta_{k}\right)\left\|B_{k} \Lambda_{n}^{k-1} u_{n}-B_{k} p\right\|^{2}
\end{aligned}
$$




$$
\begin{aligned}
& -v_{2}\left(2 \zeta_{2}-v_{2}\right)\left\|F_{2} y_{n, N}-F_{2} p\right\|^{2}-v_{1}\left(2 \zeta_{1}-v_{1}\right)\left\|F_{1} \tilde{y}_{n, N}-F_{1} \tilde{p}\right\|^{2} \\
& +2 \alpha_{n}\|(\gamma f-\mu V) p\|\left\|y_{n}-p\right\| .
\end{aligned}
$$

Since $\left(\gamma_{n}+\delta_{n}\right) \xi \leq \gamma_{n}$ for all $n \geq 0$, utilizing Lemma 2.4 we have

$$
\begin{aligned}
&\left\|x_{n+1}-p\right\|^{2} \\
&=\left\|\beta_{n}\left(x_{n}-p\right)+\gamma_{n}\left(y_{n}-p\right)+\delta_{n}\left(T y_{n}-p\right)\right\|^{2} \\
&=\left\|\beta_{n}\left(x_{n}-p\right)+\left(\gamma_{n}+\delta_{n}\right) \frac{1}{\gamma_{n}+\delta_{n}}\left[\gamma_{n}\left(y_{n}-p\right)+\delta_{n}\left(T y_{n}-p\right)\right]\right\|^{2} \\
& \leq \beta_{n}\left\|x_{n}-p\right\|^{2}+\left(\gamma_{n}+\delta_{n}\right)\left\|\frac{1}{\gamma_{n}+\delta_{n}}\left[\gamma_{n}\left(y_{n}-p\right)+\delta_{n}\left(T y_{n}-p\right)\right]\right\|^{2} \\
& \leq \beta_{n}\left\|x_{n}-p\right\|^{2}+\left(\gamma_{n}+\delta_{n}\right)\left\|y_{n}-p\right\|^{2} \\
&= \beta_{n}\left\|x_{n}-p\right\|^{2}+\left(1-\beta_{n}\right)\left\|y_{n}-p\right\|^{2} \\
& \leq \beta_{n}\left\|x_{n}-p\right\|^{2}+\left(1-\beta_{n}\right)\left[\alpha_{n} \mu \bar{\gamma}\left\|y_{n, N}-p\right\|^{2}+\left\|x_{n}-p\right\|^{2}-\left\|x_{n}-u_{n}\right\|^{2}\right. \\
&+\lambda_{k, n}\left(\lambda_{k, n}-2 \eta_{k}\right)\left\|B_{k} \Lambda_{n}^{k-1} u_{n}-B_{k} p\right\|^{2}-v_{2}\left(2 \zeta_{2}-v_{2}\right)\left\|F_{2} y_{n, N}-F_{2} p\right\|^{2} \\
&\left.-v_{1}\left(2 \zeta_{1}-v_{1}\right)\left\|F_{1} \tilde{y}_{n, N}-F_{1} \tilde{p}\right\|^{2}+2 \alpha_{n}\|(\gamma f-\mu V) p\|\left\|y_{n}-p\right\|\right] \\
& \leq\left\|x_{n}-p\right\|^{2}-\left(1-\beta_{n}\right)\left[\left\|x_{n}-u_{n}\right\|^{2}+\lambda_{k, n}\left(2 \eta_{k}-\lambda_{k, n}\right)\left\|B_{k} \Lambda_{n}^{k-1} u_{n}-B_{k} p\right\|^{2}\right. \\
&\left.+v_{2}\left(2 \zeta_{2}-v_{2}\right)\left\|F_{2} y_{n, N}-F_{2} p\right\|^{2}+v_{1}\left(2 \zeta_{1}-v_{1}\right)\left\|F_{1} \tilde{y}_{n, N}-F_{1} \tilde{p}\right\|^{2}\right] \\
&+\alpha_{n} \mu \bar{\gamma}\left\|y_{n, N}-p\right\|^{2}+2 \alpha_{n}\|(\gamma f-\mu V) p\|\left\|y_{n}-p\right\| .
\end{aligned}
$$

So, we deduce from $\left\{\beta_{n}\right\} \subset[c, d] \subset(0,1)$ and $\left\{\lambda_{k, n}\right\} \subset\left[a_{k}, b_{k}\right] \subset\left(0,2 \eta_{k}\right), k=1, \ldots, M$, that

$$
\begin{aligned}
(1-d) & {\left[\left\|x_{n}-u_{n}\right\|^{2}+\lambda_{k, n}\left(2 \eta_{k}-\lambda_{k, n}\right)\left\|B_{k} \Lambda_{n}^{k-1} u_{n}-B_{k} p\right\|^{2}\right.} \\
& \left.+v_{2}\left(2 \zeta_{2}-v_{2}\right)\left\|F_{2} y_{n, N}-F_{2} p\right\|^{2}+v_{1}\left(2 \zeta_{1}-v_{1}\right)\left\|F_{1} \tilde{y}_{n, N}-F_{1} \tilde{p}\right\|^{2}\right] \\
\leq & \left(1-\beta_{n}\right)\left[\left\|x_{n}-u_{n}\right\|^{2}+\lambda_{k, n}\left(2 \eta_{k}-\lambda_{k, n}\right)\left\|B_{k} \Lambda_{n}^{k-1} u_{n}-B_{k} p\right\|^{2}\right. \\
& \left.+v_{2}\left(2 \zeta_{2}-v_{2}\right)\left\|F_{2} y_{n, N}-F_{2} p\right\|^{2}+v_{1}\left(2 \zeta_{1}-v_{1}\right)\left\|F_{1} \tilde{y}_{n, N}-F_{1} \tilde{p}\right\|^{2}\right] \\
\leq & \left\|x_{n}-p\right\|^{2}-\left\|x_{n+1}-p\right\|^{2}+\alpha_{n} \mu \bar{\gamma}\left\|y_{n, N}-p\right\|^{2}+2 \alpha_{n}\|(\gamma f-\mu V) p\|\left\|y_{n}-p\right\| \\
\leq & \left\|x_{n}-x_{n+1}\right\|\left(\left\|x_{n}-p\right\|+\left\|x_{n+1}-p\right\|\right)+\alpha_{n} \mu \bar{\gamma}\left\|y_{n, N}-p\right\|^{2} \\
& +2 \alpha_{n}\|(\gamma f-\mu V) p\|\left\|y_{n}-p\right\| .
\end{aligned}
$$

By Propositions 3.1 and 3.2 we know that the sequences $\left\{x_{n}\right\},\left\{y_{n}\right\}$, and $\left\{y_{n, N}\right\}$ are bounded, and that $\left\{x_{n}\right\}$ is asymptotically regular. Therefore, from $\alpha_{n} \rightarrow 0$ we obtain

$$
\begin{aligned}
\lim _{n \rightarrow \infty}\left\|x_{n}-u_{n}\right\| & =\lim _{n \rightarrow \infty}\left\|F_{2} y_{n, N}-F_{2} p\right\|=\lim _{n \rightarrow \infty}\left\|F_{1} \tilde{y}_{n, N}-F_{1} \tilde{p}\right\| \\
& =\lim _{n \rightarrow \infty}\left\|B_{k} \Lambda_{n}^{k-1} u_{n}-B_{k} p\right\|=0,
\end{aligned}
$$

for each $k \in\{1, \ldots, M\}$. 
Utilizing Lemmas $2.2(\mathrm{a})$ and 2.13 , we obtain for each $k \in\{1, \ldots, M\}$

$$
\begin{aligned}
&\left\|\Lambda_{n}^{k} u_{n}-p\right\|^{2} \\
&=\left\|J_{R_{k}, \lambda_{k, n}}\left(I-\lambda_{k, n} B_{k}\right) \Lambda_{n}^{k-1} u_{n}-J_{R_{k}, \lambda_{k, n}}\left(I-\lambda_{k, n} B_{k}\right) p\right\|^{2} \\
& \leq\left\langle\left(I-\lambda_{k, n} B_{k}\right) \Lambda_{n}^{k-1} u_{n}-\left(I-\lambda_{k, n} B_{k}\right) p, \Lambda_{n}^{k} u_{n}-p\right\rangle \\
&= \frac{1}{2}\left(\left\|\left(I-\lambda_{k, n} B_{k}\right) \Lambda_{n}^{k-1} u_{n}-\left(I-\lambda_{k, n} B_{k}\right) p\right\|^{2}+\left\|\Lambda_{n}^{k} u_{n}-p\right\|^{2}\right. \\
&\left.-\left\|\left(I-\lambda_{k, n} B_{k}\right) \Lambda_{n}^{k-1} u_{n}-\left(I-\lambda_{k, n} B_{k}\right) p-\left(\Lambda_{n}^{k} u_{n}-p\right)\right\|^{2}\right) \\
& \leq \frac{1}{2}\left(\left\|\Lambda_{n}^{k-1} u_{n}-p\right\|^{2}+\left\|\Lambda_{n}^{k} u_{n}-p\right\|^{2}-\left\|\Lambda_{n}^{k-1} u_{n}-\Lambda_{n}^{k} u_{n}-\lambda_{k, n}\left(B_{k} \Lambda_{n}^{k-1} u_{n}-B_{i} p\right)\right\|^{2}\right) \\
& \leq \frac{1}{2}\left(\left\|u_{n}-p\right\|^{2}+\left\|\Lambda_{n}^{k} u_{n}-p\right\|^{2}-\left\|\Lambda_{n}^{k-1} u_{n}-\Lambda_{n}^{k} u_{n}-\lambda_{k, n}\left(B_{k} \Lambda_{n}^{k-1} u_{n}-B_{i} p\right)\right\|^{2}\right) \\
& \leq \frac{1}{2}\left(\left\|x_{n}-p\right\|^{2}+\left\|\Lambda_{n}^{k} u_{n}-p\right\|^{2}-\left\|\Lambda_{n}^{k-1} u_{n}-\Lambda_{n}^{k} u_{n}-\lambda_{k, n}\left(B_{k} \Lambda_{n}^{k-1} u_{n}-B_{k} p\right)\right\|^{2}\right),
\end{aligned}
$$

which immediately leads to

$$
\begin{aligned}
&\left\|\Lambda_{n}^{k} u_{n}-p\right\|^{2} \\
& \leq\left\|x_{n}-p\right\|^{2}-\left\|\Lambda_{n}^{k-1} u_{n}-\Lambda_{n}^{k} u_{n}-\lambda_{k, n}\left(B_{k} \Lambda_{n}^{k-1} u_{n}-B_{k} p\right)\right\|^{2} \\
&=\left\|x_{n}-p\right\|^{2}-\left\|\Lambda_{n}^{k-1} u_{n}-\Lambda_{n}^{k} u_{n}\right\|^{2}-\lambda_{k, n}^{2}\left\|B_{k} \Lambda_{n}^{k-1} u_{n}-B_{k} p\right\|^{2} \\
&+2 \lambda_{k, n}\left\langle\Lambda_{n}^{k-1} u_{n}-\Lambda_{n}^{k} u_{n}, B_{k} \Lambda_{n}^{k-1} u_{n}-B_{k} p\right\rangle \\
& \leq\left\|x_{n}-p\right\|^{2}-\left\|\Lambda_{n}^{k-1} u_{n}-\Lambda_{n}^{k} u_{n}\right\|^{2} \\
&+2 \lambda_{k, n}\left\|\Lambda_{n}^{k-1} u_{n}-\Lambda_{n}^{k} u_{n}\right\|\left\|B_{k} \Lambda_{n}^{k-1} u_{n}-B_{k} p\right\| .
\end{aligned}
$$

From (3.4), (3.20), (3.21), and (3.23) we conclude that

$$
\begin{aligned}
&\left\|x_{n+1}-p\right\|^{2} \\
& \leq \beta_{n}\left\|x_{n}-p\right\|^{2}+\left(1-\beta_{n}\right)\left\|y_{n}-p\right\|^{2} \\
& \leq \beta_{n}\left\|x_{n}-p\right\|^{2}+\left(1-\beta_{n}\right)\left[\alpha_{n} \mu \bar{\gamma}\left\|y_{n, N}-p\right\|^{2}+\left\|z_{n}-p\right\|^{2}\right. \\
&\left.+2 \alpha_{n}\|(\gamma f-\mu V) p\|\left\|y_{n}-p\right\|\right] \\
& \leq \beta_{n}\left\|x_{n}-p\right\|^{2}+\left(1-\beta_{n}\right)\left\|z_{n}-p\right\|^{2}+\alpha_{n} \mu \bar{\gamma}\left\|y_{n, N}-p\right\|^{2}+2 \alpha_{n}\|(\gamma f-\mu V) p\|\left\|y_{n}-p\right\| \\
& \leq \beta_{n}\left\|x_{n}-p\right\|^{2}+\left(1-\beta_{n}\right)\left\|y_{n, N}-p\right\|^{2}+\alpha_{n} \mu \bar{\gamma}\left\|y_{n, N}-p\right\|^{2} \\
&+2 \alpha_{n}\|(\gamma f-\mu V) p\|\left\|y_{n}-p\right\| \\
& \leq \beta_{n}\left\|x_{n}-p\right\|^{2}+\left(1-\beta_{n}\right)\left\|v_{n}-p\right\|^{2}+\alpha_{n} \mu \bar{\gamma}\left\|y_{n, N}-p\right\|^{2}+2 \alpha_{n}\|(\gamma f-\mu V) p\|\left\|y_{n}-p\right\| \\
& \leq \beta_{n}\left\|x_{n}-p\right\|^{2}+\left(1-\beta_{n}\right)\left\|\Lambda_{n}^{k} u_{n}-p\right\|^{2}+\alpha_{n} \mu \bar{\gamma}\left\|y_{n, N}-p\right\|^{2} \\
&+2 \alpha_{n}\|(\gamma f-\mu V) p\|\left\|y_{n}-p\right\| \\
& \leq \beta_{n}\left\|x_{n}-p\right\|^{2}+\left(1-\beta_{n}\right)\left[\left\|x_{n}-p\right\|^{2}-\left\|\Lambda_{n}^{k-1} u_{n}-\Lambda_{n}^{k} u_{n}\right\|^{2}\right. \\
&\left.+2 \lambda_{k, n}\left\|\Lambda_{n}^{k-1} u_{n}-\Lambda_{n}^{k} u_{n}\right\|\left\|B_{k} \Lambda_{n}^{k-1} u_{n}-B_{k} p\right\|\right]
\end{aligned}
$$




$$
\begin{aligned}
& +\alpha_{n} \mu \bar{\gamma}\left\|y_{n, N}-p\right\|^{2}+2 \alpha_{n}\|(\gamma f-\mu V) p\|\left\|y_{n}-p\right\| \\
\leq & \left\|x_{n}-p\right\|^{2}-\left(1-\beta_{n}\right)\left\|\Lambda_{n}^{k-1} u_{n}-\Lambda_{n}^{k} u_{n}\right\|^{2} \\
& +2 \lambda_{k, n}\left\|\Lambda_{n}^{k-1} u_{n}-\Lambda_{n}^{k} u_{n}\right\|\left\|B_{k} \Lambda_{n}^{k-1} u_{n}-B_{k} p\right\| \\
& +\alpha_{n} \mu \bar{\gamma}\left\|y_{n, N}-p\right\|^{2}+2 \alpha_{n}\|(\gamma f-\mu V) p\|\left\|y_{n}-p\right\|,
\end{aligned}
$$

which, together with $\left\{\beta_{n}\right\} \subset[c, d] \subset(0,1)$ and $\left\{\lambda_{k, n}\right\} \subset\left[a_{k}, b_{k}\right] \subset\left(0,2 \eta_{k}\right), k=1, \ldots, M$, yields

$$
\begin{aligned}
(1-d) & \left\|\Lambda_{n}^{k-1} u_{n}-\Lambda_{n}^{k} u_{n}\right\|^{2} \\
\leq & \left(1-\beta_{n}\right)\left\|\Lambda_{n}^{k-1} u_{n}-\Lambda_{n}^{k} u_{n}\right\|^{2} \\
\leq & \left\|x_{n}-p\right\|^{2}-\left\|x_{n+1}-p\right\|^{2}+2 \lambda_{k, n}\left\|\Lambda_{n}^{k-1} u_{n}-\Lambda_{n}^{k} u_{n}\right\|\left\|B_{k} \Lambda_{n}^{k-1} u_{n}-B_{k} p\right\| \\
& +\alpha_{n} \mu \bar{\gamma}\left\|y_{n, N}-p\right\|^{2}+2 \alpha_{n}\|(\gamma f-\mu V) p\|\left\|y_{n}-p\right\| \\
\leq & \left\|x_{n}-x_{n+1}\right\|\left(\left\|x_{n}-p\right\|+\left\|x_{n+1}-p\right\|\right)+2 b_{k}\left\|\Lambda_{n}^{k-1} u_{n}-\Lambda_{n}^{k} u_{n}\right\|\left\|B_{k} \Lambda_{n}^{k-1} u_{n}-B_{k} p\right\| \\
& +\alpha_{n} \mu \bar{\gamma}\left\|y_{n, N}-p\right\|^{2}+2 \alpha_{n}\|(\gamma f-\mu V) p\|\left\|y_{n}-p\right\| .
\end{aligned}
$$

Since $\alpha_{n} \rightarrow 0$, and $\left\{x_{n}\right\},\left\{y_{n}\right\},\left\{y_{n, N}\right\}$, and $\left\{u_{n}\right\}$ are bounded, we obtain from (3.22) and the asymptotical regularity of $\left\{x_{n}\right\}$ (due to Proposition 3.2),

$$
\lim _{n \rightarrow \infty}\left\|\Lambda_{n}^{k-1} u_{n}-\Lambda_{n}^{k} u_{n}\right\|=0, \quad \forall k \in\{1, \ldots, M\} .
$$

Therefore,

$$
\begin{aligned}
\left\|u_{n}-v_{n}\right\| & =\left\|\Lambda_{n}^{0} u_{n}-\Lambda_{n}^{M} u_{n}\right\| \\
& \leq\left\|\Lambda_{n}^{0} u_{n}-\Lambda_{n}^{1} u_{n}\right\|+\left\|\Lambda_{n}^{1} u_{n}-\Lambda_{n}^{2} u_{n}\right\|+\cdots+\left\|\Lambda_{n}^{M-1} u_{n}-\Lambda_{n}^{M} u_{n}\right\| \\
& \rightarrow 0 \quad \text { as } n \rightarrow \infty,
\end{aligned}
$$

and hence

$$
\begin{aligned}
\left\|x_{n}-v_{n}\right\| & \leq\left\|x_{n}-u_{n}\right\|+\left\|u_{n}-v_{n}\right\| \\
& \rightarrow 0 \quad \text { as } n \rightarrow \infty .
\end{aligned}
$$

Remark 3.1 By the last proposition we have $\omega_{w}\left(x_{n}\right)=\omega_{w}\left(v_{n}\right)$ and $\omega_{s}\left(x_{n}\right)=\omega_{s}\left(v_{n}\right)$, i.e., the sets of strong/weak cluster points of $\left\{x_{n}\right\}$ and $\left\{v_{n}\right\}$ coincide.

Of course, if $\beta_{n, i} \rightarrow \beta_{i} \neq 0$ as $n \rightarrow \infty$, for all indices $i$, the assumptions of Proposition 3.2 are enough to assure that

$$
\lim _{n \rightarrow \infty} \frac{\left\|x_{n+1}-x_{n}\right\|}{\beta_{n, i}}=0, \quad \forall i \in\{1, \ldots, N\} .
$$

In the next proposition, we estimate the case in which at least one sequence $\left\{\beta_{n, k_{0}}\right\}$ is a null sequence. 
Proposition 3.4 Let us suppose that $\Omega \neq \emptyset$. Let us suppose that $(\mathrm{H} 0)$ holds. Moreover, for an index $k_{0} \in\{1, \ldots, N\}, \lim _{n \rightarrow \infty} \beta_{n, k_{0}}=0$, and the following hold:

(H7) for each $i \in\{1, \ldots, N\}$ and $k \in\{1, \ldots, M\}$,

$$
\begin{aligned}
\lim _{n \rightarrow \infty} \frac{\left|\beta_{n, i}-\beta_{n-1, i}\right|}{\alpha_{n} \beta_{n, k_{0}}} & =\lim _{n \rightarrow \infty} \frac{\left|\alpha_{n}-\alpha_{n-1}\right|}{\alpha_{n} \beta_{n, k_{0}}}=\lim _{n \rightarrow \infty} \frac{\left|\beta_{n}-\beta_{n-1}\right|}{\alpha_{n} \beta_{n, k_{0}}}=\lim _{n \rightarrow \infty} \frac{\left|r_{n}-r_{n-1}\right|}{\alpha_{n} \beta_{n, k_{0}}} \\
& =\lim _{n \rightarrow \infty} \frac{1}{\alpha_{n} \beta_{n, k_{0}}}\left|\frac{\gamma_{n}}{1-\beta_{n}}-\frac{\gamma_{n-1}}{1-\beta_{n-1}}\right|=\lim _{n \rightarrow \infty} \frac{\left|\lambda_{k, n}-\lambda_{k, n-1}\right|}{\alpha_{n} \beta_{n, k_{0}}}=0 ;
\end{aligned}
$$

(H8) there exists a constant $\tau>0$ such that $\frac{1}{\alpha_{n}}\left|\frac{1}{\beta_{n, k_{0}}}-\frac{1}{\beta_{n-1, k_{0}}}\right|<\tau$ for all $n \geq 1$.

Then

$$
\lim _{n \rightarrow \infty} \frac{\left\|x_{n+1}-x_{n}\right\|}{\beta_{n, k_{0}}}
$$

Proof We start by (3.17). Dividing both terms by $\beta_{n, k_{0}}$ we have

$$
\begin{aligned}
& \frac{\left\|x_{n+1}-x_{n}\right\|}{\beta_{n, k_{0}}} \\
& \leq\left[1-(\mu \bar{\gamma}-\gamma l)(1-d) \alpha_{n}\right] \frac{|| x_{n}-x_{n-1} \|}{\beta_{n, k_{0}}}+\tilde{M}_{3}\left[\frac{\left|r_{n}-r_{n-1}\right|}{\epsilon \beta_{n, k_{0}}}+\frac{\sum_{k=1}^{M}\left|\lambda_{k, n}-\lambda_{k, n-1}\right|}{\beta_{n, k_{0}}}\right. \\
& \left.\quad+\frac{\sum_{k=1}^{N}\left|\beta_{n, k}-\beta_{n-1, k}\right|}{\beta_{n, k_{0}}}+\frac{\left|\alpha_{n}-\alpha_{n-1}\right|}{\beta_{n, k_{0}}}+\frac{\mid \frac{\gamma_{n}}{1-\beta_{n}}-\frac{\gamma_{n-1}}{1-\beta_{n-1} \mid}}{\beta_{n, k_{0}}}+\frac{\left|\beta_{n}-\beta_{n-1}\right|}{\beta_{n, k_{0}}}\right] .
\end{aligned}
$$

So, by (H8) we have

$$
\begin{aligned}
& \frac{\left\|x_{n+1}-x_{n}\right\|}{\beta_{n, k_{0}}} \\
& \leq {\left[1-(\mu \bar{\gamma}-\gamma l)(1-d) \alpha_{n}\right] \frac{\left\|x_{n}-x_{n-1}\right\|}{\beta_{n-1, k_{0}}} } \\
&+\left[1-(\mu \bar{\gamma}-\gamma l)(1-d) \alpha_{n}\right]|| x_{n}-x_{n-1} \|\left|\frac{1}{\beta_{n, k_{0}}}-\frac{1}{\beta_{n-1, k_{0}}}\right| \\
&+\widetilde{M}_{3}\left[\frac{\left|r_{n}-r_{n-1}\right|}{\epsilon \beta_{n, k_{0}}}+\frac{\sum_{k=1}^{M}\left|\lambda_{k, n}-\lambda_{k, n-1}\right|}{\beta_{n, k_{0}}}+\frac{\sum_{k=1}^{N}\left|\beta_{n, k}-\beta_{n-1, k}\right|}{\beta_{n, k_{0}}}\right. \\
&\left.+\frac{\left|\frac{\gamma_{n}}{1-\beta_{n}}-\frac{\gamma_{n-1}}{1-\beta_{n-1}}\right|}{\beta_{n, k_{0}}}+\frac{\left|\alpha_{n}-\alpha_{n-1}\right|}{\beta_{n, k_{0}}}+\frac{\left|\beta_{n}-\beta_{n-1}\right|}{\beta_{n, k_{0}}}\right] \\
& \leq {\left[1-(\mu \bar{\gamma}-\gamma l)(1-d) \alpha_{n}\right] \frac{\left\|x_{n}-x_{n-1}\right\|}{\beta_{n-1, k_{0}}}+\left\|x_{n}-x_{n-1}\right\|\left|\frac{1}{\beta_{n, k_{0}}}-\frac{1}{\beta_{n-1, k_{0}} \mid}\right| } \\
&+\widetilde{M}_{3}\left[\frac{\left|r_{n}-r_{n-1}\right|}{\epsilon \beta_{n, k_{0}}}+\frac{\sum_{k=1}^{M}\left|\lambda_{k, n}-\lambda_{k, n-1}\right|}{\beta_{n, k_{0}}}+\frac{\sum_{k=1}^{N}\left|\beta_{n, k}-\beta_{n-1, k}\right|}{\beta_{n, k_{0}}}\right. \\
&\left.+\frac{\mid \frac{\gamma_{n}}{1-\beta_{n}}-\frac{\gamma_{n-1}}{1-\beta_{n-1} \mid}}{\beta_{n, k_{0}}}+\frac{\left|\alpha_{n}-\alpha_{n-1}\right|}{\beta_{n, k_{0}}}+\frac{\left|\beta_{n}-\beta_{n-1}\right|}{\beta_{n, k_{0}}}\right] \\
& \leq {\left[1-(\mu \bar{\gamma}-\gamma l)(1-d) \alpha_{n}\right] \frac{|| x_{n}-x_{n-1} \|}{\beta_{n-1, k_{0}}}+\alpha_{n} \tau\left\|x_{n}-x_{n-1}\right\| } \\
&+\widetilde{M}_{3}\left[\frac{\left|r_{n}-r_{n-1}\right|}{\epsilon \beta_{n, k_{0}}}+\frac{\sum_{k=1}^{M}\left|\lambda_{k, n}-\lambda_{k, n-1}\right|}{\beta_{n, k_{0}}}+\frac{\sum_{k=1}^{N}\left|\beta_{n, k}-\beta_{n-1, k}\right|}{\beta_{n, k_{0}}}+\frac{\left|\frac{\gamma_{n}}{1-\beta_{n}}-\frac{\gamma_{n-1}}{1-\beta_{n-1}}\right|}{\beta_{n, k_{0}}}\right.
\end{aligned}
$$




$$
\begin{aligned}
& \left.+\frac{\left|\alpha_{n}-\alpha_{n-1}\right|}{\beta_{n, k_{0}}}+\frac{\left|\beta_{n}-\beta_{n-1}\right|}{\beta_{n, k_{0}}}\right] \\
= & {\left[1-\alpha_{n}(\mu \bar{\gamma}-\gamma l)(1-d)\right] \frac{\left\|x_{n}-x_{n-1}\right\|}{\beta_{n-1, k_{0}}}+\alpha_{n}(\mu \bar{\gamma}-\gamma l)(1-d) } \\
& \cdot \frac{1}{(\mu \bar{\gamma}-\gamma l)(1-d)}\left\{\tau\left\|x_{n}-x_{n-1}\right\|\right. \\
& +\widetilde{M}_{3}\left[\frac{\left|r_{n}-r_{n-1}\right|}{\epsilon \alpha_{n} \beta_{n, k_{0}}}+\frac{\sum_{k=1}^{M}\left|\lambda_{k, n}-\lambda_{k, n-1}\right|}{\alpha_{n} \beta_{n, k_{0}}}+\frac{\sum_{k=1}^{N}\left|\beta_{n, k}-\beta_{n-1, k}\right|}{\alpha_{n} \beta_{n, k_{0}}}+\frac{\left|\frac{\gamma_{n}}{1-\beta_{n}}-\frac{\gamma_{n-1}}{1-\beta_{n-1}}\right|}{\alpha_{n} \beta_{n, k_{0}}}\right. \\
& \left.\left.+\frac{\left|\alpha_{n}-\alpha_{n-1}\right|}{\alpha_{n} \beta_{n, k_{0}}}+\frac{\left|\beta_{n}-\beta_{n-1}\right|}{\alpha_{n} \beta_{n, k_{0}}}\right]\right\} .
\end{aligned}
$$

Therefore, utilizing Lemma 2.8, from (H0), (H7), and the asymptotical regularity of $\left\{x_{n}\right\}$ (due to Proposition 3.2), we deduce that

$$
\lim _{n \rightarrow \infty} \frac{\left\|x_{n+1}-x_{n}\right\|}{\beta_{n, k_{0}}}=0
$$

Proposition 3.5 Let us suppose that $\Omega \neq \emptyset$. Let us suppose that (H0)-(H6) hold. Then $\left\|z_{n}-y_{n, N}\right\| \rightarrow 0$ as $n \rightarrow \infty$.

Proof Let $p \in \Omega$. In terms of the firm nonexpansivity of $P_{C}$ and the $\zeta_{j}$-inverse-strong monotonicity of $F_{j}$ for $j=1,2$, we obtain from $v_{j} \in\left(0,2 \zeta_{j}\right), j=1,2$, and (3.4)

$$
\begin{aligned}
&\left\|\tilde{y}_{n, N}-\tilde{p}\right\|^{2} \\
&=\left\|P_{C}\left(I-v_{2} F_{2}\right) y_{n, N}-P_{C}\left(I-v_{2} F_{2}\right) p\right\|^{2} \\
& \leq\left\langle\left(I-v_{2} F_{2}\right) y_{n, N}-\left(I-v_{2} F_{2}\right) p, \tilde{y}_{n, N}-\tilde{p}\right\rangle \\
&= \frac{1}{2}\left[\left\|\left(I-v_{2} F_{2}\right) y_{n, N}-\left(I-v_{2} F_{2}\right) p\right\|^{2}+\left\|\tilde{y}_{n, N}-\tilde{p}\right\|^{2}\right. \\
&\left.-\left\|\left(I-v_{2} F_{2}\right) y_{n, N}-\left(I-v_{2} F_{2}\right) p-\left(\tilde{y}_{n, N}-\tilde{p}\right)\right\|^{2}\right] \\
& \leq \frac{1}{2}\left[\left\|y_{n, N}-p\right\|^{2}+\left\|\tilde{y}_{n, N}-\tilde{p}\right\|^{2}-\left\|\left(y_{n, N}-\tilde{y}_{n, N}\right)-v_{2}\left(F_{2} y_{n, N}-F_{2} p\right)-(p-\tilde{p})\right\|^{2}\right] \\
&= \frac{1}{2}\left[\left\|y_{n, N}-p\right\|^{2}+\left\|\tilde{y}_{n, N}-\tilde{p}\right\|^{2}-\left\|\left(y_{n, N}-\tilde{y}_{n, N}\right)-(p-\tilde{p})\right\|^{2}\right. \\
&\left.+2 v_{2}\left\langle\left(y_{n, N}-\tilde{y}_{n, N}\right)-(p-\tilde{p}), F_{2} y_{n, N}-F_{2} p\right\rangle-v_{2}^{2}\left\|F_{2} y_{n, N}-F_{2} p\right\|^{2}\right]
\end{aligned}
$$

and

$$
\begin{aligned}
\| z_{n}- & p \|^{2} \\
= & \left\|P_{C}\left(I-v_{1} F_{1}\right) \tilde{y}_{n, N}-P_{C}\left(I-v_{1} F_{1}\right) \tilde{p}\right\|^{2} \\
\leq & \left\langle\left(I-v_{1} F_{1}\right) \tilde{y}_{n, N}-\left(I-v_{1} F_{1}\right) \tilde{p}, z_{n}-p\right\rangle \\
= & \frac{1}{2}\left[\left\|\left(I-v_{1} F_{1}\right) \tilde{y}_{n, N}-\left(I-v_{1} F_{1}\right) \tilde{p}\right\|^{2}+\left\|z_{n}-p\right\|^{2}\right. \\
& \left.\quad-\left\|\left(I-v_{1} F_{1}\right) \tilde{y}_{n, N}-\left(I-v_{1} F_{1}\right) \tilde{p}-\left(z_{n}-p\right)\right\|^{2}\right]
\end{aligned}
$$




$$
\begin{aligned}
\leq & \frac{1}{2}\left[\left\|\tilde{y}_{n, N}-\tilde{p}\right\|^{2}+\left\|z_{n}-p\right\|^{2}-\left\|\left(\tilde{y}_{n, N}-z_{n}\right)+(p-\tilde{p})\right\|^{2}\right. \\
& \left.+2 v_{1}\left\langle F_{1} \tilde{y}_{n, N}-F_{1} \tilde{p},\left(\tilde{y}_{n, N}-z_{n}\right)+(p-\tilde{p})\right\rangle-v_{1}^{2}\left\|F_{1} \tilde{y}_{n, N}-F_{1} \tilde{p}\right\|^{2}\right] \\
\leq & \frac{1}{2}\left[\left\|y_{n, N}-p\right\|^{2}+\left\|z_{n}-p\right\|^{2}-\left\|\left(\tilde{y}_{n, N}-z_{n}\right)+(p-\tilde{p})\right\|^{2}\right. \\
& \left.+2 v_{1}\left\langle F_{1} \tilde{y}_{n, N}-F_{1} \tilde{p},\left(\tilde{y}_{n, N}-z_{n}\right)+(p-\tilde{p})\right\rangle\right] .
\end{aligned}
$$

Thus, we have

$$
\begin{aligned}
\left\|\tilde{y}_{n, N}-\tilde{p}\right\|^{2} \leq & \left\|y_{n, N}-p\right\|^{2}-\left\|\left(y_{n, N}-\tilde{y}_{n, N}\right)-(p-\tilde{p})\right\|^{2} \\
& +2 v_{2}\left\langle\left(y_{n, N}-\tilde{y}_{n, N}\right)-(p-\tilde{p}), F_{2} y_{n, N}-F_{2} p\right\rangle-v_{2}^{2}\left\|F_{2} y_{n, N}-F_{2} p\right\|^{2}
\end{aligned}
$$

and

$$
\begin{aligned}
\left\|z_{n}-p\right\|^{2} \leq & \left\|y_{n, N}-p\right\|^{2}-\left\|\left(\tilde{y}_{n, N}-z_{n}\right)+(p-\tilde{p})\right\|^{2} \\
& +2 v_{1}\left\|F_{1} \tilde{y}_{n, N}-F_{1} \tilde{p}\right\|\left\|\left(\tilde{y}_{n, N}-z_{n}\right)+(p-\tilde{p})\right\| .
\end{aligned}
$$

Consequently, from (3.4), (3.24), and (3.28), it follows that

$$
\begin{aligned}
\| x_{n+1} & -p \|^{2} \\
\leq & \beta_{n}\left\|x_{n}-p\right\|^{2}+\left(1-\beta_{n}\right)\left\|z_{n}-p\right\|^{2}+\alpha_{n} \mu \bar{\gamma}\left\|y_{n, N}-p\right\|^{2} \\
& +2 \alpha_{n}\|(\gamma f-\mu V) p\|\left\|y_{n}-p\right\| \\
\leq & \beta_{n}\left\|x_{n}-p\right\|^{2}+\left(1-\beta_{n}\right)\left\|\tilde{y}_{n, N}-\tilde{p}\right\|^{2}+\alpha_{n} \mu \bar{\gamma}\left\|y_{n, N}-p\right\|^{2} \\
& +2 \alpha_{n}\|(\gamma f-\mu V) p\|\left\|y_{n}-p\right\| \\
\leq & \beta_{n}\left\|x_{n}-p\right\|^{2}+\left(1-\beta_{n}\right)\left[\left\|y_{n, N}-p\right\|^{2}-\left\|\left(y_{n, N}-\tilde{y}_{n, N}\right)-(p-\tilde{p})\right\|^{2}\right. \\
& \left.+2 v_{2}\left\|\left(y_{n, N}-\tilde{y}_{n, N}\right)-(p-\tilde{p})\right\|\left\|F_{2} y_{n, N}-F_{2} p\right\|\right] \\
& +\alpha_{n} \mu \bar{\gamma}\left\|y_{n, N}-p\right\|^{2}+2 \alpha_{n}\|(\gamma f-\mu V) p\|\left\|y_{n}-p\right\| \\
\leq & \beta_{n}\left\|x_{n}-p\right\|^{2}+\left(1-\beta_{n}\right)\left[\left\|x_{n}-p\right\|^{2}-\left\|\left(y_{n, N}-\tilde{y}_{n, N}\right)-(p-\tilde{p})\right\|^{2}\right. \\
& \left.+2 v_{2}\left\|\left(y_{n, N}-\tilde{y}_{n, N}\right)-(p-\tilde{p})\right\|\left\|F_{2} y_{n, N}-F_{2} p\right\|\right] \\
& +\alpha_{n} \mu \bar{\gamma}\left\|y_{n, N}-p\right\|^{2}+2 \alpha_{n}\|(\gamma f-\mu V) p\|\left\|y_{n}-p\right\| \\
\leq & \left\|x_{n}-p\right\|^{2}-\left(1-\beta_{n}\right)\left\|\left(y_{n, N}-\tilde{y}_{n, N}\right)-(p-\tilde{p})\right\|^{2} \\
& +2 v_{2}\left\|\left(y_{n, N}-\tilde{y}_{n, N}\right)-(p-\tilde{p})\right\|\left\|F_{2} y_{n, N}-F_{2} p\right\| \\
& +\alpha_{n} \mu \bar{\gamma}\left\|y_{n, N}-p\right\|^{2}+2 \alpha_{n}\|(\gamma f-\mu V) p\|\left\|y_{n}-p\right\|,
\end{aligned}
$$

which yields

$$
\begin{aligned}
& (1-d)\left\|\left(y_{n, N}-\tilde{y}_{n, N}\right)-(p-\tilde{p})\right\|^{2} \\
& \quad \leq\left(1-\beta_{n}\right)\left\|\left(y_{n, N}-\tilde{y}_{n, N}\right)-(p-\tilde{p})\right\|^{2} \\
& \quad \leq\left\|x_{n}-p\right\|^{2}-\left\|x_{n+1}-p\right\|^{2}+2 v_{2}\left\|\left(y_{n, N}-\tilde{y}_{n, N}\right)-(p-\tilde{p})\right\|\left\|F_{2} y_{n, N}-F_{2} p\right\|
\end{aligned}
$$




$$
\begin{aligned}
& +\alpha_{n} \mu \bar{\gamma}\left\|y_{n, N}-p\right\|^{2}+2 \alpha_{n}\|(\gamma f-\mu V) p\|\left\|y_{n}-p\right\| \\
\leq & \left\|x_{n}-x_{n+1}\right\|\left(\left\|x_{n}-p\right\|+\left\|x_{n+1}-p\right\|\right)+2 v_{2}\left\|\left(y_{n, N}-\tilde{y}_{n, N}\right)-(p-\tilde{p})\right\|\left\|F_{2} y_{n, N}-F_{2} p\right\| \\
& +\alpha_{n} \mu \bar{\gamma}\left\|y_{n, N}-p\right\|^{2}+2 \alpha_{n}\|(\gamma f-\mu V) p\|\left\|y_{n}-p\right\| .
\end{aligned}
$$

Since $\lim _{n \rightarrow \infty} \alpha_{n}=0, \lim _{n \rightarrow \infty}\left\|x_{n+1}-x_{n}\right\|=0$, and $\left\{x_{n}\right\},\left\{y_{n}\right\},\left\{y_{n, N}\right\}$, and $\left\{\tilde{y}_{n, N}\right\}$ are bounded, we deduce from (3.22) that

$$
\lim _{n \rightarrow \infty}\left\|\left(y_{n, N}-\tilde{y}_{n, N}\right)-(p-\tilde{p})\right\|=0
$$

Furthermore, from (3.4), (3.24), and (3.29), it follows that

$$
\begin{aligned}
\| x_{n+1}- & p \|^{2} \\
\leq & \beta_{n}\left\|x_{n}-p\right\|^{2}+\left(1-\beta_{n}\right)\left\|z_{n}-p\right\|^{2}+\alpha_{n} \mu \bar{\gamma}\left\|y_{n, N}-p\right\|^{2} \\
& +2 \alpha_{n}\|(\gamma f-\mu V) p\|\left\|y_{n}-p\right\| \\
\leq & \beta_{n}\left\|x_{n}-p\right\|^{2}+\left(1-\beta_{n}\right)\left[\left\|y_{n, N}-p\right\|^{2}-\left\|\left(\tilde{y}_{n, N}-z_{n}\right)+(p-\tilde{p})\right\|^{2}\right. \\
& \left.+2 v_{1}\left\|F_{1} \tilde{y}_{n, N}-F_{1} \tilde{p}\right\|\left\|\left(\tilde{y}_{n, N}-z_{n}\right)+(p-\tilde{p})\right\|\right] \\
& +\alpha_{n} \mu \bar{\gamma}\left\|y_{n, N}-p\right\|^{2}+2 \alpha_{n}\|(\gamma f-\mu V) p\|\left\|y_{n}-p\right\| \\
\leq & \beta_{n}\left\|x_{n}-p\right\|^{2}+\left(1-\beta_{n}\right)\left[\left\|x_{n}-p\right\|^{2}-\left\|\left(\tilde{y}_{n, N}-z_{n}\right)+(p-\tilde{p})\right\|^{2}\right. \\
& \left.+2 v_{1}\left\|F_{1} \tilde{y}_{n, N}-F_{1} \tilde{p}\right\|\left\|\left(\tilde{y}_{n, N}-z_{n}\right)+(p-\tilde{p})\right\|\right] \\
& +\alpha_{n} \mu \bar{\gamma}\left\|y_{n, N}-p\right\|^{2}+2 \alpha_{n}\|(\gamma f-\mu V) p\|\left\|y_{n}-p\right\| \\
\leq & \left\|x_{n}-p\right\|^{2}-\left(1-\beta_{n}\right)\left\|\left(\tilde{y}_{n, N}-z_{n}\right)+(p-\tilde{p})\right\|^{2} \\
& +2 v_{1}\left\|F_{1} \tilde{y}_{n, N}-F_{1} \tilde{p}\right\|\left\|\left(\tilde{y}_{n, N}-z_{n}\right)+(p-\tilde{p})\right\| \\
& +\alpha_{n} \mu \bar{\gamma}\left\|y_{n, N}-p\right\|^{2}+2 \alpha_{n}\|(\gamma f-\mu V) p\|\left\|y_{n}-p\right\|,
\end{aligned}
$$

which leads to

$$
\begin{aligned}
(1-d) & \left\|\left(\tilde{y}_{n, N}-z_{n}\right)+(p-\tilde{p})\right\|^{2} \\
\leq & \left(1-\beta_{n}\right)\left\|\left(\tilde{y}_{n, N}-z_{n}\right)+(p-\tilde{p})\right\|^{2} \\
\leq & \left\|x_{n}-p\right\|^{2}-\left\|x_{n+1}-p\right\|^{2}+2 v_{1}\left\|F_{1} \tilde{y}_{n, N}-F_{1} \tilde{p}\right\|\left\|\left(\tilde{y}_{n, N}-z_{n}\right)+(p-\tilde{p})\right\| \\
& +\alpha_{n} \mu \bar{\gamma}\left\|y_{n, N}-p\right\|^{2}+2 \alpha_{n}\|(\gamma f-\mu V) p\|\left\|y_{n}-p\right\| \\
\leq & \left\|x_{n}-x_{n+1}\right\|\left(\left\|x_{n}-p\right\|+\left\|x_{n+1}-p\right\|\right)+2 v_{1}\left\|F_{1} \tilde{y}_{n, N}-F_{1} \tilde{p}\right\|\left\|\left(\tilde{y}_{n, N}-z_{n}\right)+(p-\tilde{p})\right\| \\
& +\alpha_{n} \mu \bar{\gamma}\left\|y_{n, N}-p\right\|^{2}+2 \alpha_{n}\|(\gamma f-\mu V) p\|\left\|y_{n}-p\right\| .
\end{aligned}
$$

Since $\lim _{n \rightarrow \infty} \alpha_{n}=0, \lim _{n \rightarrow \infty}\left\|x_{n+1}-x_{n}\right\|=0$, and $\left\{x_{n}\right\},\left\{z_{n}\right\},\left\{y_{n}\right\},\left\{y_{n, N}\right\}$, and $\left\{\tilde{y}_{n, N}\right\}$ are bounded, we deduce from (3.22) that

$$
\lim _{n \rightarrow \infty}\left\|\left(\tilde{y}_{n, N}-z_{n}\right)+(p-\tilde{p})\right\|=0 .
$$


Note that

$$
\left\|y_{n, N}-z_{n}\right\| \leq\left\|\left(y_{n, N}-\tilde{y}_{n, N}\right)-(p-\tilde{p})\right\|+\left\|\left(\tilde{y}_{n, N}-z_{n}\right)+(p-\tilde{p})\right\| .
$$

Hence from (3.30) and (3.31) we get

$$
\lim _{n \rightarrow \infty}\left\|y_{n, N}-z_{n}\right\|=\lim _{n \rightarrow \infty}\left\|y_{n, N}-G y_{n, N}\right\|=0 .
$$

Proposition 3.6 Let us suppose that $\Omega \neq \emptyset$. Let us suppose that $0<\liminf _{n \rightarrow \infty} \beta_{n, i} \leq$ $\limsup _{n \rightarrow \infty} \beta_{n, i}<1$ for each $i=1, \ldots, N$. Moreover, suppose that (H0)-(H6) are satisfied. Then $\lim _{n \rightarrow \infty}\left\|S_{i} v_{n}-v_{n}\right\|=0$ for each $i=1, \ldots, N$ provided $\left\|T y_{n}-y_{n}\right\| \rightarrow$ as $n \rightarrow \infty$.

Proof First of all, observe that

$$
\begin{aligned}
x_{n+1}-x_{n} & =\gamma_{n}\left(y_{n}-x_{n}\right)+\delta_{n}\left(T y_{n}-x_{n}\right) \\
& =\gamma_{n}\left(y_{n}-x_{n}\right)+\delta_{n}\left(T y_{n}-y_{n}\right)+\delta_{n}\left(y_{n}-x_{n}\right) \\
& =\left(\gamma_{n}+\delta_{n}\right)\left(y_{n}-x_{n}\right)+\delta_{n}\left(T y_{n}-y_{n}\right) \\
& =\left(1-\beta_{n}\right)\left(y_{n}-x_{n}\right)+\delta_{n}\left(T y_{n}-y_{n}\right) .
\end{aligned}
$$

By Proposition 3.2 we know that $\left\{x_{n}\right\}$ is asymptotically regular. Hence we have

$$
\left(1-\beta_{n}\right)\left\|y_{n}-x_{n}\right\|=\left\|x_{n+1}-x_{n}-\delta_{n}\left(T y_{n}-y_{n}\right)\right\| \leq\left\|x_{n+1}-x_{n}\right\|+\delta_{n}\left\|T y_{n}-y_{n}\right\|,
$$

which, together with $\left\|T y_{n}-y_{n}\right\| \rightarrow 0$, implies that

$$
\lim _{n \rightarrow \infty}\left\|x_{n}-y_{n}\right\|=0
$$

Let us show that for each $i \in\{1, \ldots, N\}$, one has $\left\|S_{i} v_{n}-y_{n, i-1}\right\| \rightarrow 0$ as $n \rightarrow \infty$. Let $p \in \Omega$. When $i=N$, by Lemma 2.2(b) we have from (3.3), (3.4), and (3.20)

$$
\begin{aligned}
\left\|y_{n}-p\right\|^{2} \leq & \alpha_{n} \mu \bar{\gamma}\left\|y_{n, N}-p\right\|^{2}+\left\|z_{n}-p\right\|^{2}+2 \alpha_{n}\|(\gamma f-\mu V) p\|\left\|y_{n}-p\right\| \\
\leq & \alpha_{n} \mu \bar{\gamma}\left\|y_{n, N}-p\right\|^{2}+2 \alpha_{n}\|(\gamma f-\mu V) p\|\left\|y_{n}-p\right\|+\left\|y_{n, N}-p\right\|^{2} \\
= & \alpha_{n} \mu \bar{\gamma}\left\|y_{n, N}-p\right\|^{2}+2 \alpha_{n}\|(\gamma f-\mu V) p\|\left\|y_{n}-p\right\|+\beta_{n, N}\left\|S_{N} v_{n}-p\right\|^{2} \\
& +\left(1-\beta_{n, N}\right)\left\|y_{n, N-1}-p\right\|^{2}-\beta_{n, N}\left(1-\beta_{n, N}\right)\left\|S_{N} v_{n}-y_{n, N-1}\right\|^{2} \\
\leq & \alpha_{n} \mu \bar{\gamma}\left\|y_{n, N}-p\right\|^{2}+2 \alpha_{n}\|(\gamma f-\mu V) p\|\left\|y_{n}-p\right\|+\beta_{n, N}\left\|v_{n}-p\right\|^{2} \\
& +\left(1-\beta_{n, N}\right)\left\|v_{n}-p\right\|^{2}-\beta_{n, N}\left(1-\beta_{n, N}\right)\left\|S_{N} v_{n}-y_{n, N-1}\right\|^{2} \\
= & \alpha_{n} \mu \bar{\gamma}\left\|y_{n, N}-p\right\|^{2}+2 \alpha_{n}\|(\gamma f-\mu V) p\|\left\|y_{n}-p\right\|+\left\|v_{n}-p\right\|^{2} \\
& -\beta_{n, N}\left(1-\beta_{n, N}\right)\left\|S_{N} v_{n}-y_{n, N-1}\right\|^{2} \\
\leq & \alpha_{n} \mu \bar{\gamma}\left\|y_{n, N}-p\right\|^{2}+2 \alpha_{n}\|(\gamma f-\mu V) p\|\left\|y_{n}-p\right\|+\left\|x_{n}-p\right\|^{2} \\
& -\beta_{n, N}\left(1-\beta_{n, N}\right)\left\|S_{N} v_{n}-y_{n, N-1}\right\|^{2} .
\end{aligned}
$$


So, we have

$$
\begin{aligned}
& \beta_{n, N}\left(1-\beta_{n, N}\right)\left\|S_{N} v_{n}-y_{n, N-1}\right\|^{2} \\
& \quad \leq \alpha_{n} \mu \bar{\gamma}\left\|y_{n, N}-p\right\|^{2}+2 \alpha_{n}\|(\gamma f-\mu V) p\|\left\|y_{n}-p\right\|+\left\|x_{n}-p\right\|^{2}-\left\|y_{n}-p\right\|^{2} \\
& \quad \leq \alpha_{n} \mu \bar{\gamma}\left\|y_{n, N}-p\right\|^{2}+2 \alpha_{n}\|(\gamma f-\mu V) p\|\left\|y_{n}-p\right\|+\left\|x_{n}-y_{n}\right\|\left(\left\|x_{n}-p\right\|+\left\|y_{n}-p\right\|\right) .
\end{aligned}
$$

Since $\alpha_{n} \rightarrow 0,0<\liminf _{n \rightarrow \infty} \beta_{n, N} \leq \limsup _{n \rightarrow \infty} \beta_{n, N}<1$, and $\lim _{n \rightarrow \infty}\left\|x_{n}-y_{n}\right\|=0$ (due to (3.33)), it is well known that $\left\{\left\|S_{N} v_{n}-y_{n, N-1}\right\|\right\}$ is a null sequence.

Let $i \in\{1, \ldots, N-1\}$. Then one has

$$
\begin{aligned}
\left\|y_{n}-p\right\|^{2} \leq & \alpha_{n} \mu \bar{\gamma}\left\|y_{n, N}-p\right\|^{2}+2 \alpha_{n}\|(\gamma f-\mu V) p\|\left\|y_{n}-p\right\|+\left\|y_{n, N}-p\right\|^{2} \\
\leq & \alpha_{n} \mu \bar{\gamma}\left\|y_{n, N}-p\right\|^{2}+2 \alpha_{n}\|(\gamma f-\mu V) p\|\left\|y_{n}-p\right\|+\beta_{n, N}\left\|S_{N} v_{n}-p\right\|^{2} \\
& +\left(1-\beta_{n, N}\right)\left\|y_{n, N-1}-p\right\|^{2} \\
\leq & \alpha_{n} \mu \bar{\gamma}\left\|y_{n, N}-p\right\|^{2}+2 \alpha_{n}\|(\gamma f-\mu V) p\|\left\|y_{n}-p\right\|+\beta_{n, N}\left\|x_{n}-p\right\|^{2} \\
& +\left(1-\beta_{n, N}\right)\left\|y_{n, N-1}-p\right\|^{2} \\
\leq & \alpha_{n} \mu \bar{\gamma}\left\|y_{n, N}-p\right\|^{2}+2 \alpha_{n}\|(\gamma f-\mu V) p\|\left\|y_{n}-p\right\|+\beta_{n, N}\left\|x_{n}-p\right\|^{2} \\
& +\left(1-\beta_{n, N}\right)\left[\beta_{n, N-1}\left\|S_{N-1} v_{n}-p\right\|^{2}+\left(1-\beta_{n, N-1}\right)\left\|y_{n, N-2}-p\right\|^{2}\right] \\
\leq & \alpha_{n} \mu \bar{\gamma}\left\|y_{n, N}-p\right\|^{2}+2 \alpha_{n}\|(\gamma f-\mu V) p\|\left\|y_{n}-p\right\| \\
& +\left(\beta_{n, N}+\left(1-\beta_{n, N}\right) \beta_{n, N-1}\right)\left\|x_{n}-p\right\|^{2}+\prod_{k=N-1}^{N}\left(1-\beta_{n, k}\right)\left\|y_{n, N-2}-p\right\|^{2},
\end{aligned}
$$

and so, after $(N-i+1)$ iterations,

$$
\begin{aligned}
\left\|y_{n}-p\right\|^{2} \leq & \alpha_{n} \mu \bar{\gamma}\left\|y_{n, N}-p\right\|^{2}+2 \alpha_{n}\|(\gamma f-\mu V) p\|\left\|y_{n}-p\right\| \\
& +\left(\beta_{n, N}+\sum_{j=i+2}^{N}\left(\prod_{l=j}^{N}\left(1-\beta_{n, l}\right)\right) \beta_{n, j-1}\right)\left\|x_{n}-p\right\|^{2}+\prod_{k=i+1}^{N}\left(1-\beta_{n, k}\right)\left\|y_{n, i}-p\right\|^{2} \\
\leq & \alpha_{n} \mu \bar{\gamma}\left\|y_{n, N}-p\right\|^{2}+2 \alpha_{n}\|(\gamma f-\mu V) p\|\left\|y_{n}-p\right\| \\
& +\left(\beta_{n, N}+\sum_{j=i+2}^{N}\left(\prod_{l=j}^{N}\left(1-\beta_{n, l}\right)\right) \beta_{n, j-1}\right)\left\|x_{n}-p\right\|^{2} \\
& +\prod_{k=i+1}^{N}\left(1-\beta_{n, k}\right)\left[\beta_{n, i}\left\|S_{i} u_{n}-p\right\|^{2}\right. \\
& \left.+\left(1-\beta_{n, i}\right)\left\|y_{n, i-1}-p\right\|^{2}-\beta_{n, i}\left(1-\beta_{n, i}\right)\left\|S_{i} v_{n}-y_{n, i-1}\right\|^{2}\right] \\
\leq & \alpha_{n} \mu \bar{\gamma}\left\|y_{n, N}-p\right\|^{2}+2 \alpha_{n}\|(\gamma f-\mu V) p\|\left\|y_{n}-p\right\|+\left\|x_{n}-p\right\|^{2} \\
& -\beta_{n, i} \prod_{k=i}^{N}\left(1-\beta_{n, k}\right)\left\|S_{i} v_{n}-y_{n, i-1}\right\|^{2} .
\end{aligned}
$$


Again we obtain

$$
\begin{aligned}
& \beta_{n, i} \prod_{k=i}^{N}\left(1-\beta_{n, k}\right)\left\|S_{i} v_{n}-y_{n, i-1}\right\|^{2} \\
& \quad \leq \alpha_{n} \mu \bar{\gamma}\left\|y_{n, N}-p\right\|^{2}+2 \alpha_{n}\|(\gamma f-\mu V) p\|\left\|y_{n}-p\right\|+\left\|x_{n}-p\right\|^{2}-\left\|y_{n}-p\right\|^{2} \\
& \quad \leq \alpha_{n} \mu \bar{\gamma}\left\|y_{n, N}-p\right\|^{2}+2 \alpha_{n}\|(\gamma f-\mu V) p\|\left\|y_{n}-p\right\|+\left\|x_{n}-y_{n}\right\|\left(\left\|x_{n}-p\right\|+\left\|y_{n}-p\right\|\right) .
\end{aligned}
$$

Since $\alpha_{n} \rightarrow 0,0<\liminf _{n \rightarrow \infty} \beta_{n, i} \leq \limsup _{n \rightarrow \infty} \beta_{n, i}<1$ for each $i=1, \ldots, N-1$, and $\lim _{n \rightarrow \infty}\left\|x_{n}-y_{n}\right\|=0$ (due to (3.33)), it is well known that

$$
\lim _{n \rightarrow \infty}\left\|S_{i} v_{n}-y_{n, i-1}\right\|=0
$$

Obviously for $i=1$, we have $\left\|S_{1} u_{n}-u_{n}\right\| \rightarrow 0$.

To conclude, we have

$$
\left\|S_{2} v_{n}-v_{n}\right\| \leq\left\|S_{2} v_{n}-y_{n, 1}\right\|+\left\|y_{n, 1}-v_{n}\right\|=\left\|S_{2} v_{n}-y_{n, 1}\right\|+\beta_{n, 1}\left\|S_{1} v_{n}-v_{n}\right\|
$$

from which $\left\|S_{2} v_{n}-v_{n}\right\| \rightarrow 0$. Thus by induction $\left\|S_{i} v_{n}-v_{n}\right\| \rightarrow 0$ for all $i=2, \ldots, N$ since it is enough to observe that

$$
\begin{aligned}
\left\|S_{i} v_{n}-v_{n}\right\| & \leq\left\|S_{i} v_{n}-y_{n, i-1}\right\|+\left\|y_{n, i-1}-S_{i-1} v_{n}\right\|+\left\|S_{i-1} v_{n}-v_{n}\right\| \\
& \leq\left\|S_{i} v_{n}-y_{n, i-1}\right\|+\left(1-\beta_{n, i-1}\right)\left\|S_{i-1} v_{n}-y_{n, i-2}\right\|+\left\|S_{i-1} v_{n}-v_{n}\right\| .
\end{aligned}
$$

Remark 3.2 As an example, we consider $M=1, N=2$, and the sequences:
(a) $\lambda_{1, n}=\eta_{1}-\frac{1}{n}, \forall n>\frac{1}{\eta_{1}}$;
(b) $\alpha_{n}=\frac{1}{\sqrt{n}}, r_{n}=2-\frac{1}{n}, \forall n>1$;
(c) $\beta_{n}=\beta_{n, 1}=\frac{1}{2}-\frac{1}{n}, \beta_{n, 2}=\frac{1}{2}-\frac{1}{n^{2}}, \forall n>2$.

They satisfy the hypotheses on the parameter sequences in Proposition 3.6.

Proposition 3.7 Let us suppose that $\Omega \neq \emptyset$ and $\beta_{n, i} \rightarrow \beta_{i}$ for all $i$ as $n \rightarrow \infty$. Suppose there exists $k \in\{1, \ldots, N\}$ such that $\beta_{n, k} \rightarrow 0$ as $n \rightarrow \infty$. Let $k_{0} \in\{1, \ldots, N\}$ be the largest index such that $\beta_{n, k_{0}} \rightarrow 0$ as $n \rightarrow \infty$. Suppose that

(i) $\frac{\alpha_{n}}{\beta_{n, k_{0}}} \rightarrow 0$ as $n \rightarrow \infty$;

(ii) if $i \leq k_{0}$ and $\beta_{n, i} \rightarrow 0$ then $\frac{\beta_{n, k_{0}}}{\beta_{n, i}} \rightarrow 0$ as $n \rightarrow \infty$;

(iii) if $\beta_{n, i} \rightarrow \beta_{i} \neq 0$ then $\beta_{i}$ lies in $(0,1)$.

Moreover, suppose that (H0), (H7), and (H8) hold. Then $\lim _{n \rightarrow \infty}\left\|S_{i} v_{n}-v_{n}\right\|=0$ for each $i=1, \ldots, N$ provided $\left\|T y_{n}-y_{n}\right\| \rightarrow 0$ as $n \rightarrow \infty$.

Proof First of all we note that if (H7) holds then also (H1)-(H6) are satisfied. So $\left\{x_{n}\right\}$ is asymptotically regular.

Let $k_{0}$ be as in the hypotheses. As in Proposition 3.6, for every index $i \in\{1, \ldots, N\}$ such that $\beta_{n, i} \rightarrow \beta_{i} \neq 0$ (which leads to $0<\liminf _{n \rightarrow \infty} \beta_{n, i} \leq \limsup _{n \rightarrow \infty} \beta_{n, i}<1$ ), one has $\| S_{i} v_{n}-$ $y_{n, i-1} \| \rightarrow 0$ as $n \rightarrow \infty$. 
For all the other indices $i \leq k_{0}$, we can prove that $\left\|S_{i} v_{n}-y_{n, i-1}\right\| \rightarrow 0$ as $n \rightarrow \infty$ in a similar manner. By the relation (due to (3.21) and (3.34))

$$
\begin{aligned}
\left\|x_{n+1}-p\right\|^{2} \leq & \beta_{n}\left\|x_{n}-p\right\|^{2}+\left(1-\beta_{n}\right)\left\|y_{n}-p\right\|^{2} \\
\leq & \beta_{n}\left\|x_{n}-p\right\|^{2}+\left(1-\beta_{n}\right)\left[\alpha_{n} \mu \bar{\gamma}\left\|y_{n, N}-p\right\|^{2}+2 \alpha_{n}\|(\gamma f-\mu V) p\|\left\|y_{n}-p\right\|\right. \\
& \left.+\left\|x_{n}-p\right\|^{2}-\beta_{n, i} \prod_{k=i}^{N}\left(1-\beta_{n, k}\right)\left\|S_{i} v_{n}-y_{n, i-1}\right\|^{2}\right] \\
\leq & \left\|x_{n}-p\right\|^{2}+\alpha_{n} \mu \bar{\gamma}\left\|y_{n, N}-p\right\|^{2}+2 \alpha_{n}\|(\gamma f-\mu V) p\|\left\|y_{n}-p\right\| \\
& -\left(1-\beta_{n}\right) \beta_{n, i} \prod_{k=i}^{N}\left(1-\beta_{n, k}\right)\left\|S_{i} v_{n}-y_{n, i-1}\right\|^{2}
\end{aligned}
$$

we immediately obtain

$$
\begin{aligned}
(1-d) & \prod_{k=i}^{N}\left(1-\beta_{n, k}\right)\left\|S_{i} v_{n}-y_{n, i-1}\right\|^{2} \\
\leq & \left(1-\beta_{n}\right) \prod_{k=i}^{N}\left(1-\beta_{n, k}\right)\left\|S_{i} v_{n}-y_{n, i-1}\right\|^{2} \\
\leq & \frac{\alpha_{n}}{\beta_{n, i}}\left[\mu \bar{\gamma}\left\|y_{n, N}-p\right\|^{2}+2\|(\gamma f-\mu V) p\|\left\|y_{n}-p\right\|\right] \\
& +\frac{\left\|x_{n}-x_{n+1}\right\|}{\beta_{n, i}}\left(\left\|x_{n}-p\right\|+\left\|x_{n+1}-p\right\|\right) .
\end{aligned}
$$

By Proposition 3.4 or by hypothesis (ii) on the sequences, we have

$$
\frac{\left\|x_{n}-x_{n+1}\right\|}{\beta_{n, i}}=\frac{\left\|x_{n}-x_{n+1}\right\|}{\beta_{n, k_{0}}} \cdot \frac{\beta_{n, k_{0}}}{\beta_{n, i}} \rightarrow 0 .
$$

So, the conclusion follows.

Remark 3.3 Let us consider $M=1, N=3$, and the following sequences:

(a) $\alpha_{n}=\frac{1}{n^{1 / 2}}, r_{n}=2-\frac{1}{n^{2}}, \forall n>1$;

(b) $\lambda_{1, n}=\eta_{1}-\frac{1}{n^{2}}, \forall n>\frac{1}{\eta_{1}^{1 / 2}}$;

(c) $\beta_{n, 1}=\frac{1}{n^{1 / 4}}, \beta_{n}=\beta_{n, 2}=\frac{1}{2}-\frac{1}{n^{2}}, \beta_{n, 3}=\frac{1}{n^{1 / 3}}, \forall n>1$.

It is easy to see that all hypotheses (i)-(iii), (H0), (H7), and (H8) of Proposition 3.7 are satisfied.

Remark 3.4 Under the hypotheses of Proposition 3.7, analogously to Proposition 3.6, one can see that

$$
\lim _{n \rightarrow \infty}\left\|S_{i} v_{n}-y_{n, i-1}\right\|=0, \quad \forall i \in\{2, \ldots, N\}
$$

Corollary 3.1 Let us suppose that the hypotheses of either Proposition 3.6 or Proposition 3.7 are satisfied. Then $\omega_{w}\left(x_{n}\right)=\omega_{w}\left(v_{n}\right)=\omega_{w}\left(y_{n, 1}\right), \omega_{s}\left(x_{n}\right)=\omega_{s}\left(v_{n}\right)=\omega_{s}\left(y_{n, 1}\right)$, and $\omega_{w}\left(x_{n}\right) \subset \Omega$. 
Proof By Remark 3.1, we have $\omega_{w}\left(x_{n}\right)=\omega_{w}\left(v_{n}\right)$ and $\omega_{s}\left(x_{n}\right)=\omega_{s}\left(v_{n}\right)$. Note that by Remark 3.4,

$$
\lim _{n \rightarrow \infty}\left\|S_{N} v_{n}-y_{n, N-1}\right\|=0 .
$$

In the meantime, it is well known that

$$
\lim _{n \rightarrow \infty}\left\|S_{N} v_{n}-v_{n}\right\|=\lim _{n \rightarrow \infty}\left\|v_{n}-x_{n}\right\|=\lim _{n \rightarrow \infty}\left\|x_{n}-y_{n}\right\|=0 .
$$

Hence we have

$$
\lim _{n \rightarrow \infty}\left\|S_{N} v_{n}-y_{n}\right\|=0
$$

Furthermore, it follows from (3.1) that

$$
\lim _{n \rightarrow \infty}\left\|y_{n, N}-y_{n, N-1}\right\|=\lim _{n \rightarrow \infty} \beta_{n, N}\left\|S_{N} v_{n}-y_{n, N-1}\right\|=0,
$$

which, together with $\lim _{n \rightarrow \infty}\left\|S_{N} v_{n}-y_{n, N-1}\right\|=0$, yields

$$
\lim _{n \rightarrow \infty}\left\|S_{N} v_{n}-y_{n, N}\right\|=0
$$

Combining (3.35) and (3.36), we conclude that

$$
\lim _{n \rightarrow \infty}\left\|y_{n}-y_{n, N}\right\|=0
$$

which, together with $\lim _{n \rightarrow \infty}\left\|x_{n}-y_{n}\right\|=0$, leads to

$$
\lim _{n \rightarrow \infty}\left\|x_{n}-y_{n, N}\right\|=0
$$

Now we observe that

$$
\left\|x_{n}-y_{n, 1}\right\| \leq\left\|x_{n}-v_{n}\right\|+\left\|y_{n, 1}-v_{n}\right\|=\left\|x_{n}-v_{n}\right\|+\beta_{n, 1}\left\|S_{1} v_{n}-v_{n}\right\| .
$$

By Propositions 3.3 and 3.6, $\left\|x_{n}-v_{n}\right\| \rightarrow 0$ and $\left\|S_{1} v_{n}-v_{n}\right\| \rightarrow 0$ as $n \rightarrow \infty$, and hence

$$
\lim _{n \rightarrow \infty}\left\|x_{n}-y_{n, 1}\right\|=0
$$

So we get $\omega_{w}\left(x_{n}\right)=\omega_{w}\left(y_{n, 1}\right)$ and $\omega_{s}\left(x_{n}\right)=\omega_{s}\left(y_{n, 1}\right)$.

Let $p \in \omega_{w}\left(x_{n}\right)$. Then there exists a subsequence $\left\{x_{n_{i}}\right\}$ of $\left\{x_{n}\right\}$ such that $x_{n_{i}} \rightarrow p$. Since $p \in \omega_{w}\left(v_{n}\right)$, by Proposition 3.6 and Lemma 2.5 (demiclosedness principle), we have $p \in$ Fix $\left(S_{i}\right)$ for each $i=1, \ldots, N$, i.e., $p \in \bigcap_{i=1}^{N} \operatorname{Fix}\left(S_{i}\right)$. Taking into account $p \in \omega_{w}\left(y_{n, N}\right)$ (due to (3.38)) and $\left\|y_{n, N}-G y_{n, N}\right\| \rightarrow 0$ (due to (3.32)), by Lemma 2.5 (demiclosedness principle) we know that $p \in \operatorname{Fix}(G)=: \Xi$. Also, since $p \in \omega_{w}\left(y_{n}\right)$ (due to $\left\|x_{n}-y_{n}\right\| \rightarrow 0$ ), in terms of $\left\|T y_{n}-y_{n}\right\| \rightarrow 0$ and Lemma 2.3 (demiclosedness principle), we get $p \in \operatorname{Fix}(T)$. Moreover, by Lemma 2.11 and Proposition 3.3 we know that $p \in \operatorname{GMEP}(\Theta, h)$. Next we prove that $p \in \bigcap_{m=1}^{M} \mathrm{I}\left(B_{m}, R_{m}\right)$. As a matter of fact, from (3.22) and (3.25) we know that 
$u_{n_{i}} \rightarrow p$ and $\Lambda_{n_{i}}^{m} u_{n_{i}} \rightarrow p$ for each $m=1, \ldots, M$. Since $B_{m}$ is $\eta_{m}$-inverse-strongly monotone, $B_{m}$ is a monotone and Lipschitz-continuous mapping. It follows from Lemma 2.16 that $R_{m}+B_{m}$ is maximal monotone. Let $(v, g) \in G\left(R_{m}+B_{m}\right)$, i.e., $g-B_{m} v \in R_{m} v$. Again, since $\Lambda_{n}^{m} u_{n}=J_{R_{m}, \lambda_{m, n}}\left(I-\lambda_{m, n} B_{m}\right) \Lambda_{n}^{m-1} u_{n}, n \geq 0, m \in\{1,2, \ldots, N\}$, we have

$$
\Lambda_{n}^{m-1} u_{n}-\lambda_{m, n} B_{m} \Lambda_{n}^{m-1} u_{n} \in\left(I+\lambda_{m, n} R_{m}\right) \Lambda_{n}^{m} u_{n}
$$

that is,

$$
\frac{1}{\lambda_{m, n}}\left(\Lambda_{n}^{m-1} u_{n}-\Lambda_{n}^{m} u_{n}-\lambda_{m, n} B_{m} \Lambda_{n}^{m-1} u_{n}\right) \in R_{m} \Lambda_{n}^{m} u_{n}
$$

In terms of the monotonicity of $R_{m}$, we get

$$
\left\langle v-\Lambda_{n}^{m} u_{n}, g-B_{m} v-\frac{1}{\lambda_{m, n}}\left(\Lambda_{n}^{m-1} u_{n}-\Lambda_{n}^{m} u_{n}-\lambda_{m, n} B_{m} \Lambda_{n}^{m-1} u_{n}\right)\right\rangle \geq 0
$$

and hence

$$
\begin{aligned}
\langle v- & \left.\Lambda_{n}^{m} u_{n}, g\right\rangle \\
& \geq\left\langle v-\Lambda_{n}^{m} u_{n}, B_{m} v+\frac{1}{\lambda_{m, n}}\left(\Lambda_{n}^{m-1} u_{n}-\Lambda_{n}^{m} u_{n}-\lambda_{m, n} B_{m} \Lambda_{n}^{m-1} u_{n}\right)\right\rangle \\
& =\left\langle v-\Lambda_{n}^{m} u_{n}, B_{m} v-B_{m} \Lambda_{n}^{m} u_{n}+B_{m} \Lambda_{n}^{m} u_{n}-B_{m} \Lambda_{n}^{m-1} u_{n}+\frac{1}{\lambda_{m, n}}\left(\Lambda_{n}^{m-1} u_{n}-\Lambda_{n}^{m} u_{n}\right)\right\rangle \\
& \geq\left\langle v-\Lambda_{n}^{m} u_{n}, B_{m} \Lambda_{n}^{m} u_{n}-B_{m} \Lambda_{n}^{m-1} u_{n}\right\rangle+\left\langle v-\Lambda_{n}^{m} u_{n}, \frac{1}{\lambda_{m, n}}\left(\Lambda_{n}^{m-1} u_{n}-\Lambda_{n}^{m} u_{n}\right)\right\rangle .
\end{aligned}
$$

In particular,

$$
\begin{aligned}
\left\langle v-\Lambda_{n_{i}}^{m} u_{n_{i}}, g\right\rangle \geq & \left\langle v-\Lambda_{n_{i}}^{m} u_{n_{i}}, B_{m} \Lambda_{n_{i}}^{m} u_{n_{i}}-B_{m} \Lambda_{n_{i}}^{m-1} u_{n_{i}}\right\rangle \\
& +\left\langle v-\Lambda_{n_{i}}^{m} u_{n_{i}}, \frac{1}{\lambda_{m, n_{i}}}\left(\Lambda_{n_{i}}^{m-1} u_{n_{i}}-\Lambda_{n_{i}}^{m} u_{n_{i}}\right)\right\rangle
\end{aligned}
$$

Since $\left\|\Lambda_{n}^{m} u_{n}-\Lambda_{n}^{m-1} u_{n}\right\| \rightarrow 0$ (due to (3.25)) and $\left\|B_{m} \Lambda_{n}^{m} u_{n}-B_{m} \Lambda_{n}^{m-1} u_{n}\right\| \rightarrow 0$ (due to the Lipschitz-continuity of $\left.B_{m}\right)$, we conclude from $\Lambda_{n_{i}}^{m} u_{n_{i}} \rightarrow p$ and $\left\{\lambda_{m, n}\right\} \subset\left[a_{m}, b_{m}\right] \subset$ $\left(0,2 \eta_{m}\right)$ that

$$
\lim _{i \rightarrow \infty}\left\langle v-\Lambda_{n_{i}}^{m} u_{n_{i}}, g\right\rangle=\langle v-p, g\rangle \geq 0
$$

It follows from the maximal monotonicity of $B_{m}+R_{m}$ that $0 \in\left(R_{m}+B_{m}\right) w$, i.e., $p \in$ $\mathrm{I}\left(B_{m}, R_{m}\right)$. Therefore, $p \in \bigcap_{m=1}^{M} \mathrm{I}\left(B_{m}, R_{m}\right)$. Consequently, $p \in \operatorname{Fix}(T) \cap \bigcap_{i=1}^{N} \operatorname{Fix}\left(S_{i}\right) \cap$ $\operatorname{GMEP}(\Theta, h) \cap \bigcap_{m=1}^{M} \mathrm{I}\left(B_{m}, R_{m}\right)=: \Omega$.

Theorem 3.1 Let us suppose that $\Omega \neq \emptyset$. Let $\left\{\alpha_{n}\right\},\left\{\beta_{n, i}\right\}, i=1, \ldots, N$, be sequences in $(0,1)$ such that $0<\liminf _{n \rightarrow \infty} \beta_{n, i} \leq \lim \sup _{n \rightarrow \infty} \beta_{n, i}<1$ for each index $i$. Moreover, let us suppose that (H0)-(H6) hold. Then the sequences $\left\{u_{n}\right\},\left\{v_{n}\right\},\left\{x_{n}\right\}$, and $\left\{y_{n}\right\}$ defined by scheme (3.1), 
all converge strongly to $x^{*}=P_{\Omega}(I-(\mu V-\gamma f)) x^{*}$ if and only if $\left\|y_{n}-T y_{n}\right\| \rightarrow 0$ as $n \rightarrow \infty$, where $x^{*}=P_{\Omega}(I-(\mu V-\gamma f)) x^{*}$ is the unique solution of the VIP

$$
\left\langle(\gamma f-\mu V) x^{*}, x-x^{*}\right\rangle \leq 0, \quad \forall x \in \Omega
$$

or, equivalently, the unique solution of the minimization problem

$$
\min _{x \in \Omega} \frac{\mu}{2}\langle V x, x\rangle-\Psi(x)
$$

where $\Psi$ is a potential function for $\gamma f$.

Proof First of all, we note that $V$ is a $\bar{\gamma}$-strongly positive bounded linear operator on $H$ and $f: H \rightarrow H$ is an $l$-Lipschitz-continuous mapping with $0 \leq \gamma l<\mu \bar{\gamma}$. It is clear that

$$
\langle(\mu V-\gamma f) x-(\mu V-\gamma f) y, x-y\rangle \geq(\mu \bar{\gamma}-\gamma l)\|x-y\|^{2}, \quad \forall x, y \in H .
$$

Hence we deduce that $\mu V-\gamma f$ is $(\mu \bar{\gamma}-\gamma l)$-strongly monotone. In the meantime, it is easy to see that $\mu V-\gamma f$ is $(\mu\|V\|+\gamma l)$-Lipschitz-continuous with constant $\mu\|V\|+\gamma l>0$. Thus, there exists a unique solution $x^{*}$ in $\Omega$ to the VIP (3.39). Equivalently, $x^{*}$ is the unique solution of the minimization problem (3.40).

Now, observe that there exists a subsequence $\left\{x_{n_{i}}\right\}$ of $\left\{x_{n}\right\}$ such that

$$
\limsup _{n \rightarrow \infty}\left\langle(\gamma f-\mu V) x^{*}, x_{n}-x^{*}\right\rangle=\lim _{i \rightarrow \infty}\left\langle(\gamma f-\mu V) x^{*}, x_{n_{i}}-x^{*}\right\rangle
$$

Since $\left\{x_{n_{i}}\right\}$ is bounded, there exists a subsequence $\left\{x_{n_{i j}}\right\}$ of $\left\{x_{n_{i}}\right\}$ which converges weakly to some $p \in H$. Without loss of generality, we may assume that $x_{n_{i}} \rightarrow p$. Then by Corollary 3.1, we get $p \in \omega_{w}\left(x_{n}\right) \subset \Omega$. Hence, from (3.39) and (3.41), we have

$$
\limsup _{n \rightarrow \infty}\left\langle(\gamma f-\mu V) x^{*}, x_{n}-x^{*}\right\rangle=\left\langle(\gamma f-\mu V) x^{*}, p-x^{*}\right\rangle \leq 0 .
$$

Since (H1)-(H6) hold, the sequence $\left\{x_{n}\right\}$ is asymptotically regular (according to Proposition 3.2). In terms of (3.33) and Proposition 3.3, $\left\|x_{n}-y_{n}\right\| \rightarrow 0$ and $\left\|x_{n}-v_{n}\right\| \rightarrow 0$ as $n \rightarrow \infty$.

Let us show that $\left\|x_{n}-x^{*}\right\| \rightarrow 0$ as $n \rightarrow \infty$. Indeed, putting $p=x^{*}$, we deduce from (3.4), (3.20), and (3.21) that

$$
\begin{aligned}
&\left\|x_{n+1}-x^{*}\right\|^{2} \\
& \leq \beta_{n}\left\|x_{n}-x^{*}\right\|^{2}+\left(1-\beta_{n}\right)\left\|y_{n}-x^{*}\right\|^{2} \\
& \leq \beta_{n}\left\|x_{n}-x^{*}\right\|^{2}+\left(1-\beta_{n}\right)\left[\alpha_{n} \mu \bar{\gamma} \frac{(\gamma l)^{2}}{(\mu \bar{\gamma})^{2}}\left\|y_{n, N}-x^{*}\right\|^{2}+\left(1-\alpha_{n} \mu \bar{\gamma}\right)\left\|z_{n}-x^{*}\right\|^{2}\right. \\
&\left.+2 \alpha_{n}\left((\gamma f-\mu V) x^{*}, y_{n}-x^{*}\right\rangle\right] \\
& \leq \beta_{n}\left\|x_{n}-x^{*}\right\|^{2}+\left(1-\beta_{n}\right)\left[\alpha_{n} \frac{(\gamma l)^{2}}{\mu \bar{\gamma}}\left\|x_{n}-x^{*}\right\|^{2}+\left(1-\alpha_{n} \mu \bar{\gamma}\right)\left\|x_{n}-x^{*}\right\|^{2}\right.
\end{aligned}
$$




$$
\begin{aligned}
& \left.+2 \alpha_{n}\left\langle(\gamma f-\mu V) x^{*}, y_{n}-x^{*}\right\rangle\right] \\
= & \beta_{n}\left\|x_{n}-x^{*}\right\|^{2}+\left(1-\beta_{n}\right)\left[\left(1-\alpha_{n} \frac{(\mu \bar{\gamma})^{2}-(\gamma l)^{2}}{\mu \bar{\gamma}}\right)\left\|x_{n}-x^{*}\right\|^{2}\right. \\
& \left.+2 \alpha_{n}\left\langle(\gamma f-\mu V) x^{*}, y_{n}-x^{*}\right\rangle\right] \\
= & \left(1-\alpha_{n}\left(1-\beta_{n}\right) \frac{(\mu \bar{\gamma})^{2}-(\gamma l)^{2}}{\mu \bar{\gamma}}\right)\left\|x_{n}-x^{*}\right\|^{2}+2 \alpha_{n}\left(1-\beta_{n}\right)\left\langle(\gamma f-\mu V) x^{*}, y_{n}-x^{*}\right\rangle \\
\leq & \left(1-\alpha_{n}\left(1-\beta_{n}\right) \frac{(\mu \bar{\gamma})^{2}-(\gamma l)^{2}}{\mu \bar{\gamma}}\right)\left\|x_{n}-x^{*}\right\|^{2} \\
& +\alpha_{n}\left(1-\beta_{n}\right) \frac{(\mu \bar{\gamma})^{2}-(\gamma l)^{2}}{\mu \bar{\gamma}} \cdot \frac{2 \mu \bar{\gamma}}{(\mu \bar{\gamma})^{2}-(\gamma l)^{2}}\left\langle(\gamma f-\mu V) x^{*}, y_{n}-x^{*}\right\rangle .
\end{aligned}
$$

Since $\sum_{n=0}^{\infty} \alpha_{n}=\infty,\left\{\beta_{n}\right\} \subset[c, d] \subset(0,1)$ and $\left\|x_{n}-y_{n}\right\| \rightarrow 0$, we obtain $\sum_{n=0}^{\infty} \alpha_{n}(1-$ $\left.\beta_{n}\right) \frac{(\mu \bar{\gamma})^{2}-(\gamma l)^{2}}{\mu \bar{\gamma}} \geq \sum_{n=0}^{\infty} \alpha_{n}(1-d) \frac{(\mu \bar{\gamma})^{2}-(\gamma l)^{2}}{\mu \bar{\gamma}}=\infty$ and

$$
\begin{aligned}
& \limsup _{n \rightarrow \infty} \frac{2 \mu \bar{\gamma}}{(\mu \bar{\gamma})^{2}-(\gamma l)^{2}}\left\langle(\gamma f-\mu V) x^{*}, y_{n}-x^{*}\right\rangle \\
& \quad=\limsup _{n \rightarrow \infty} \frac{2 \mu \bar{\gamma}}{(\mu \bar{\gamma})^{2}-(\gamma l)^{2}}\left(\left\langle(\gamma f-\mu V) x^{*}, x_{n}-x^{*}\right\rangle+\left\langle(\gamma f-\mu V) x^{*}, y_{n}-x_{n}\right\rangle\right) \\
& \quad=\limsup _{n \rightarrow \infty} \frac{2 \mu \bar{\gamma}}{(\mu \bar{\gamma})^{2}-(\gamma l)^{2}}\left\langle(\gamma f-\mu V) x^{*}, x_{n}-x^{*}\right\rangle \leq 0
\end{aligned}
$$

(due to (3.42)). Applying Lemma 2.8 to (3.43), we infer that the sequence $\left\{x_{n}\right\}$ converges strongly to $x^{*}$. This completes the proof.

In a similar way, we can conclude to another theorem, as follows.

Theorem 3.2 Let us suppose that $\Omega \neq \emptyset$. Let $\left\{\alpha_{n}\right\},\left\{\beta_{n, i}\right\}, i=1, \ldots, N$, be sequences in $(0,1)$ such that $\beta_{n, i} \rightarrow \beta_{i}$ for each index $i$ as $n \rightarrow \infty$. Suppose that there exists $k \in\{1, \ldots, N\}$ for which $\beta_{n, k} \rightarrow 0$ as $n \rightarrow \infty$. Let $k_{0} \in\{1, \ldots, N\}$ the largest index for which $\beta_{n, k_{0}} \rightarrow 0$. Moreover, let us suppose that (H0), (H7), and (H8) hold and

(i) $\frac{\alpha_{n}}{\beta_{n, k_{0}}} \rightarrow 0$ as $n \rightarrow \infty$;

(ii) if $i \leq k_{0}$ and $\beta_{n, i} \rightarrow \beta_{i}$ then $\frac{\beta_{n, k_{0}}}{\beta_{n, i}} \rightarrow 0$ as $n \rightarrow \infty$;

(iii) if $\beta_{n, i} \rightarrow \beta_{i} \neq 0$ then $\beta_{i}$ lies in $(0,1)$.

Then the sequences $\left\{u_{n}\right\},\left\{v_{n}\right\},\left\{x_{n}\right\}$, and $\left\{y_{n}\right\}$ defined by scheme (3.1) all converge strongly to $x^{*}=P_{\Omega}(I-(\mu V-\gamma f)) x^{*}$ if and only if $\left\|y_{n}-T y_{n}\right\| \rightarrow 0$ as $n \rightarrow \infty$, where $x^{*}=P_{\Omega}(I-$ $(\mu V-\gamma f)) x^{*}$ is the unique solution of the VIP

$$
\left\langle(\gamma f-\mu V) x^{*}, x-x^{*}\right\rangle \leq 0, \quad \forall x \in \Omega
$$

or, equivalently, the unique solution of the minimization problem

$$
\min _{x \in \Omega} \frac{\mu}{2}\langle V x, x\rangle-\Psi(x)
$$

where $\Psi$ is a potential function for $\gamma f$. 
Remark 3.5 According to the above argument process for Theorems 3.1 and 3.2, we can readily see that if in scheme (3.1), the iterative step $y_{n}=\alpha_{n} \gamma f\left(y_{n, N}\right)+\left(I-\alpha_{n} \mu V\right) G y_{n, N}$ is replaced by the iterative one $y_{n}=\alpha_{n} \gamma f\left(x_{n}\right)+\left(I-\alpha_{n} \mu V\right) G y_{n, N}$, then Theorems 3.1 and 3.2 remain valid.

Remark 3.6 Theorems 3.1 and 3.2 improve, extend, supplement, and develop [13], Theorems 3.1 and 3.2 and [33], Theorems 3.12 and 3.13 in the following aspects.

(i) The multi-step iterative scheme (3.1) of [13] is extended to develop our relaxed extragradient iterative scheme (3.1) by virtue of Korpelevich's extragradient method and the strongly positive bounded linear operator approach. The iterative scheme (3.1) is based on Korpelevich's extragradient method, the viscosity approximation method [42] (see also [43]), Mann's iterative method, and the strongly positive bounded linear operator approach.

(ii) The argument techniques in our Theorems 3.1 and 3.2 are very different from the techniques in [13], Theorems 3.1 and 3.2 and [33], Theorems 3.12 and 3.13, because we make use of the properties of strict pseudocontractions (see Lemmas 2.3 and 2.4), the ones of resolvent operators and maximal monotone mappings (see Remark 2.1 and Lemmas 2.12-2.16), the ones of the resolvent operator associated with $\Theta$ and $h$ (see Lemmas 2.9-2.11), the fixed point problem $x^{*}=G x^{*}(\Leftrightarrow$ GSVI (1.6)) (see Proposition 2.2), and the ones of strongly positive boundedness linear operators (see Lemma 2.7).

(iii) The problem of finding an element of $\operatorname{Fix}(T) \cap \bigcap_{i=1}^{N} \operatorname{Fix}\left(S_{i}\right) \cap \operatorname{GMEP}(\Theta, h) \cap$ $\bigcap_{k=1}^{M} \mathrm{I}\left(B_{k}, R_{k}\right) \cap \Xi$ in our Theorems 3.1 and 3.2 is more general and more subtle than the one of finding an element of $\operatorname{Fix}(T) \cap \bigcap_{i=1}^{N} \operatorname{Fix}\left(S_{i}\right) \cap \operatorname{GMEP}(\Theta, h)$ in [33], Theorems 3.12 and 3.13 (where $T$ is a nonexpansive mapping) and the one of finding an element of $\operatorname{Fix}(T) \cap \bigcap_{i=1}^{N} \operatorname{Fix}\left(S_{i}\right) \cap \operatorname{GMEP}(\Theta, h) \cap \Xi$ in [13], Theorems 3.1 and 3.2 (where $T$ is a strict pseudocontraction).

(iv) Our Theorems 3.1 and 3.2 generalizes from the nonexpansive mapping $T$ to the strict pseudocontraction $T$ and extend [33], Theorems 3.12 and 3.13 to the setting of GSVI (1.6), hierarchical minimization (3.40) and finitely many variational inclusions for maximal monotone and inverse-strongly monotone mappings. In the meantime, our Theorems 3.1 and 3.2 extend [13], Theorems 3.1 and 3.2 to the setting of hierarchical minimization (3.40) and finitely many variational inclusions for maximal monotone and inversestrongly monotone mappings.

\section{Applications}

For a given nonlinear mapping $A: C \rightarrow H$, we consider the variational inequality problem (VIP) of finding $\bar{x} \in C$ such that

$$
\langle A \bar{x}, y-\bar{x}\rangle \geq 0, \quad \forall y \in C .
$$

We will indicate by $\operatorname{VI}(C, A)$ the set of solutions of the VIP (4.1).

Recall that if $u$ is a point in $C$, then the following relation holds:

$$
u \in \operatorname{VI}(C, A) \quad \Leftrightarrow \quad u=P_{C}(I-\lambda A) u, \quad \forall \lambda>0 .
$$


In the meantime, it is easy to see that the following relation holds:

$$
\text { GSVI (1.6) with } F_{2}=0 \quad \Leftrightarrow \quad \text { VIP (4.1) with } A=F_{1} \text {. }
$$

An operator $A: C \rightarrow H$ is said to be an $\alpha$-inverse-strongly monotone operator if there exists a constant $\alpha>0$ such that

$$
\langle A x-A y, x-y\rangle \geq \alpha\|A x-A y\|^{2}, \quad \forall x, y \in C .
$$

As an example, we recall that the $\alpha$-inverse-strongly monotone operators are firmly nonexpansive mappings if $\alpha \geq 1$ and that every $\alpha$-inverse-strongly monotone operator is also $\frac{1}{\alpha}$-Lipschitz-continuous (see [45]).

Let us observe also that, if $A$ is $\alpha$-inverse-strongly monotone, the mappings $P_{C}(I-\lambda A)$ are nonexpansive for all $\lambda \in(0,2 \alpha]$ since they are compositions of nonexpansive mappings (see p.419 in [45]).

Let us consider $\tilde{S}_{1}, \ldots, \tilde{S}_{K}$ to be a finite number of nonexpansive self-mappings on $C$ and $A_{1}, \ldots, A_{N}$ to be a finite number of $\alpha$-inverse-strongly monotone operators. Let $T: H \rightarrow H$ be a $\xi$-strict pseudocontraction on $C$ with fixed points. Let us consider the mixed problem of finding $x^{*} \in \operatorname{Fix}(T) \cap \operatorname{GMEP}(\Theta, h) \cap \Xi \cap \bigcap_{k=1}^{M} \mathrm{I}\left(B_{k}, R_{k}\right)$ such that

$$
\left\{\begin{array}{l}
\left\langle\left(I-\tilde{S}_{1}\right) x^{*}, y-x^{*}\right\rangle \geq 0, \quad \forall y \in \operatorname{Fix}(T) \cap \operatorname{GMEP}(\Theta, h) \cap \Xi \cap \bigcap_{k=1}^{M} \mathrm{I}\left(B_{k}, R_{k}\right), \\
\left\langle\left(I-\tilde{S}_{2}\right) x^{*}, y-x^{*}\right\rangle \geq 0, \quad \forall y \in \operatorname{Fix}(T) \cap \operatorname{GMEP}(\Theta, h) \cap \Xi \cap \bigcap_{k=1}^{M} \mathrm{I}\left(B_{k}, R_{k}\right), \\
\cdots \\
\left\langle\left(I-\tilde{S}_{K}\right) x^{*}, y-x^{*}\right\rangle \geq 0, \quad \forall y \in \operatorname{Fix}(T) \cap \operatorname{GMEP}(\Theta, h) \cap \Xi \cap \bigcap_{k=1}^{M} \mathrm{I}\left(B_{k}, R_{k}\right), \\
\left\langle A_{1} x^{*}, y-x^{*}\right\rangle \geq 0, \quad \forall y \in C, \\
\left\langle A_{2} x^{*}, y-x^{*}\right\rangle \geq 0, \quad \forall y \in C, \\
\cdots \\
\left\langle A_{N} x^{*}, y-x^{*}\right\rangle \geq 0, \quad \forall y \in C .
\end{array}\right.
$$

Let us call (SVI) the set of solutions of the $(N+K)$-system. This problem is equivalent to finding a common fixed point of $T,\left\{P_{\mathrm{Fix}(T) \cap G M E P(\Theta, h) \cap \Xi \cap \bigcap_{k=1}^{M} \mathrm{I}\left(B_{k}, R_{k}\right)} \tilde{S}_{i}\right\}_{i=1}^{K},\left\{P_{C}\left(I-\lambda A_{i}\right)\right\}_{i=1}^{N}$. So we claim that the following holds.

Theorem 4.1 Let us suppose that $\Omega=\operatorname{Fix}(T) \cap(\mathrm{SVI}) \cap \operatorname{GMEP}(\Theta, h) \cap \Xi \cap \bigcap_{k=1}^{M} \mathrm{I}\left(B_{k}, R_{k}\right) \neq$ $\emptyset$. Fix $\lambda>0$. Let $\left\{\alpha_{n}\right\},\left\{\beta_{n, i}\right\}, i=1, \ldots,(K+N)$, be sequences in $(0,1)$ such that $0<$ $\liminf _{n \rightarrow \infty} \beta_{n, i} \leq \limsup _{n \rightarrow \infty} \beta_{n, i}<1$ for all indices $i$. Moreover, let us suppose that (H0)(H6) hold. Then the sequences $\left\{u_{n}\right\},\left\{v_{n}\right\},\left\{x_{n}\right\}$, and $\left\{y_{n}\right\}$ explicitly defined by scheme

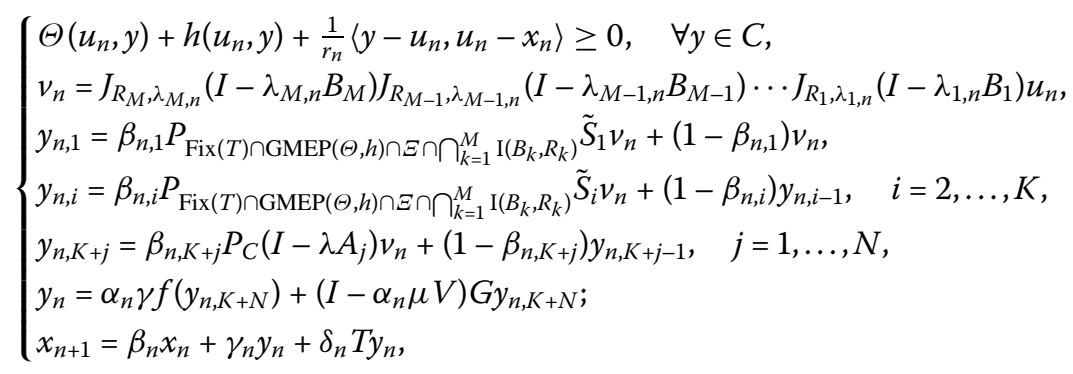


all converge strongly to $x^{*}=P_{\Omega}(I-(\mu V-\gamma f)) x^{*}$ if and only if $\left\|y_{n}-T y_{n}\right\| \rightarrow 0$ as $n \rightarrow \infty$, where $x^{*}=P_{\Omega}(I-(\mu V-\gamma f)) x^{*}$ is the unique solution of the VIP

$$
\left\langle(\gamma f-\mu V) x^{*}, x-x^{*}\right\rangle \leq 0, \quad \forall x \in \Omega
$$

or, equivalently, the unique solution of the minimization problem

$$
\min _{x \in \Omega} \frac{\mu}{2}\langle V x, x\rangle-\Psi(x)
$$

where $\Psi$ is a potential function for $\gamma f$.

Theorem 4.2 Let us suppose that $\Omega \neq \emptyset$. Fix $\lambda>0$. Let $\left\{\alpha_{n}\right\},\left\{\beta_{n, i}\right\}, i=1, \ldots,(K+N)$, be sequences in $(0,1)$ and $\beta_{n, i} \rightarrow \beta_{i}$ for all $i$ as $n \rightarrow \infty$. Suppose that there exists $k \in\{1, \ldots, K+$ $N\}$ such that $\beta_{n, k} \rightarrow 0$ as $n \rightarrow \infty$. Let $k_{0} \in\{1, \ldots, K+N\}$ be the largest index for which $\beta_{n, k_{0}} \rightarrow 0$. Moreover, let us suppose that (H0), (H7), and (H8) hold and

(i) $\frac{\alpha_{n}}{\beta_{n, k_{0}}} \rightarrow 0$ as $n \rightarrow \infty$;

(ii) if $i \leq k_{0}$ and $\beta_{n, i} \rightarrow 0$ then $\frac{\beta_{n, k_{0}}}{\beta_{n, i}} \rightarrow 0$ as $n \rightarrow \infty$;

(iii) if $\beta_{n, i} \rightarrow \beta_{i} \neq 0$ then $\beta_{i}$ lies in $(0,1)$.

Then the sequences $\left\{u_{n}\right\},\left\{v_{n}\right\},\left\{x_{n}\right\}$, and $\left\{y_{n}\right\}$ explicitly defined by scheme (4.4) all converge strongly to $x^{*}=P_{\Omega}(I-(\mu V-\gamma f)) x^{*}$ if and only if $\left\|y_{n}-T y_{n}\right\| \rightarrow 0$ as $n \rightarrow \infty$, where $x^{*}=$ $P_{\Omega}(I-(\mu V-\gamma f)) x^{*}$ is the unique solution of the VIP

$$
\left\langle(\gamma f-\mu V) x^{*}, x-x^{*}\right\rangle \leq 0, \quad \forall x \in \Omega
$$

or, equivalently, the unique solution of the minimization problem

$$
\min _{x \in \Omega} \frac{\mu}{2}\langle V x, x\rangle-\Psi(x)
$$

where $\Psi$ is a potential function for $\gamma f$.

Remark 4.1 If in system (4.3), $F_{1}=F_{2}=A_{1}=\cdots=A_{N}=0, B_{1}=\cdots=B_{M}=R_{1}=\cdots=$ $R_{M}=0$, and $T$ is a nonexpansive mapping, we obtain a system of hierarchical fixed point problems introduced by Mainge and Moudafi $[26,28]$.

On the other hand, if $S: C \rightarrow C$ is a $\kappa$-strictly pseudocontractive mapping, that is, there exists a constant $\kappa \in[0,1)$ such that

$$
\|S x-S y\|^{2} \leq\|x-y\|^{2}+\kappa\|(I-S) x-(I-S) y\|^{2}, \quad \forall x, y \in C,
$$

then $A=I-S$ is $\frac{1-\kappa}{2}$-inverse-strongly monotone; see [52].

Utilizing Theorems 3.1 and 3.2, we also give two strong convergence theorems for finding a common element of the solution set $\operatorname{GMEP}(\Theta, h)$ of $\operatorname{GMEP}(1.2)$, the solution set $\bigcap_{k=1}^{M} \mathrm{I}\left(B_{k}, R_{k}\right)$ of finitely many variational inclusions and the common fixed point set $\bigcap_{i=1}^{N} \operatorname{Fix}\left(S_{i}\right) \cap \operatorname{Fix}(S)$ of finitely many nonexpansive mappings $S_{i}: C \rightarrow C, i=1, \ldots, N$, and a $\kappa$-strictly pseudocontractive mapping $S$. 
Theorem 4.3 Let $v_{1} \in(0,1-\kappa)$. Let us suppose that $\Omega=\bigcap_{i=1}^{N} \operatorname{Fix}\left(S_{i}\right) \cap \operatorname{Fix}(S) \cap \operatorname{GMEP}(\Theta$, $h) \cap \bigcap_{k=1}^{M} \mathrm{I}\left(B_{k}, R_{k}\right) \neq \emptyset$. Let $\left\{\alpha_{n}\right\},\left\{\beta_{n, i}\right\}, i=1, \ldots, N$, be sequences in $(0,1)$ such that $0<$ $\liminf _{n \rightarrow \infty} \beta_{n, i} \leq \limsup _{n \rightarrow \infty} \beta_{n, i}<1$ for all indices $i$. Moreover, let us suppose that we have (H0)-(H6) with $\gamma_{n}=0, \forall n \geq 0$. Then the sequences $\left\{u_{n}\right\},\left\{v_{n}\right\},\left\{x_{n}\right\}$, and $\left\{y_{n}\right\}$ generated explicitly by

$$
\left\{\begin{array}{l}
\Theta\left(u_{n}, y\right)+h\left(u_{n}, y\right)+\frac{1}{r_{n}}\left\langle y-u_{n}, u_{n}-x_{n}\right\rangle \geq 0, \quad \forall y \in C \\
v_{n}=J_{R_{M}, \lambda_{M, n}}\left(I-\lambda_{M, n} B_{M}\right) J_{R_{M-1}, \lambda_{M-1, n}}\left(I-\lambda_{M-1, n} B_{M-1}\right) \cdots J_{R_{1}, \lambda_{1, n}}\left(I-\lambda_{1, n} B_{1}\right) u_{n} \\
y_{n, 1}=\beta_{n, 1} S_{1} v_{n}+\left(1-\beta_{n, 1}\right) v_{n} \\
y_{n, i}=\beta_{n, i} S_{i} v_{n}+\left(1-\beta_{n, i}\right) y_{n, i-1}, \quad i=2, \ldots, N \\
y_{n}=\alpha_{n} f\left(y_{n, N}\right)+\left(1-\alpha_{n}\right)\left(\left(1-v_{1}\right) y_{n, N}+v_{1} S y_{n, N}\right) \\
x_{n+1}=\beta_{n} x_{n}+\left(1-\beta_{n}\right) y_{n}, \quad \forall n \geq 0
\end{array}\right.
$$

all converge strongly to $x^{*}=P_{\Omega}(I-(\mu V-\gamma f)) x^{*}$, which is the unique solution of the VIP

$$
\left\langle(\gamma f-\mu V) x^{*}, x-x^{*}\right\rangle \leq 0, \quad \forall x \in \Omega
$$

or, equivalently, the unique solution of the minimization problem

$$
\min _{x \in \Omega} \frac{\mu}{2}\langle V x, x\rangle-\Psi(x)
$$

where $\Psi$ is a potential function for $\gamma f$.

Proof In Theorem 3.1, put $F_{1}=A=I-S$ and $F_{2}=0$. Then $A$ is $\frac{1-\kappa}{2}$-inverse-strongly monotone. Hence we deduce that $\operatorname{Fix}(S)=\operatorname{VI}(C, A)=\Gamma$ and

$$
\begin{aligned}
G y_{n, N} & =P_{C}\left(I-v_{1} F_{1}\right) P_{C}\left(I-v_{2} F_{2}\right) y_{n, N} \\
& =P_{C}\left(I-v_{1} F_{1}\right) y_{n, N} \\
& =\left(1-v_{1}\right) y_{n, N}+v_{1} S y_{n, N} .
\end{aligned}
$$

Thus, in terms of Theorem 3.1, we obtain the desired result.

Theorem 4.4 Let $v_{1} \in(0,1-\kappa)$. Let us suppose that $\Omega=\bigcap_{i=1}^{N} \operatorname{Fix}\left(S_{i}\right) \cap \operatorname{Fix}(S) \cap \operatorname{GMEP}(\Theta$, $h) \cap \bigcap_{k=1}^{M} \mathrm{I}\left(B_{k}, R_{k}\right) \neq \emptyset$. Let $\left\{\alpha_{n}\right\},\left\{\beta_{n, i}\right\}, i=1, \ldots, N$, be sequences in $(0,1)$ such that $\beta_{n, i} \rightarrow \beta_{i}$ for all $i$ as $n \rightarrow \infty$. Suppose that there exists $k \in\{1, \ldots, N\}$ for which $\beta_{n, k} \rightarrow 0$ as $n \rightarrow \infty$. Let $k_{0} \in\{1, \ldots, N\}$ be the largest index for which $\beta_{n, k_{0}} \rightarrow 0$. Moreover, let us suppose that we have (H0), (H7), and (H8) with $\gamma_{n}=0, \forall n \geq 0$, and

(i) $\frac{\alpha_{n}}{\beta_{n, k_{0}}} \rightarrow 0$ as $n \rightarrow \infty$;

(ii) if $i \leq k_{0}$ and $\beta_{n, i} \rightarrow 0$ then $\frac{\beta_{n, k_{0}}}{\beta_{n, i}} \rightarrow 0$ as $n \rightarrow \infty$;

(iii) if $\beta_{n, i} \rightarrow \beta_{i} \neq 0$ then $\beta_{i}$ lies in $(0,1)$.

Then the sequences $\left\{u_{n}\right\},\left\{v_{n}\right\},\left\{x_{n}\right\}$, and $\left\{y_{n}\right\}$, generated explicitly by (4.5), all converge strongly to $x^{*}=P_{\Omega}(I-(\mu V-\gamma f)) x^{*}$, which is the unique solution of the VIP

$$
\left\langle(\gamma f-\mu V) x^{*}, x-x^{*}\right\rangle \leq 0, \quad \forall x \in \Omega
$$


or, equivalently, the unique solution of the minimization problem

$$
\min _{x \in \Omega} \frac{\mu}{2}\langle V x, x\rangle-\Psi(x)
$$

where $\Psi$ is a potential function for $\gamma f$.

\section{Competing interests}

The authors declare that they have no competing interests.

\section{Authors' contributions}

All authors read and approved the final manuscript.

\section{Author details}

${ }^{1}$ Department of Mathematics, Shanghai Normal University, Shanghai, 200234, China. ${ }^{2}$ Scientific Computing Key Laboratory of Shanghai Universities, Shanghai, 200234, China. ${ }^{3}$ Center for Fundamental Science, Kaohsiung Medical University, Kaohsiung, 807, Taiwan. ${ }^{4}$ Center of Nonlinear Analysis and Optimization, Kaohsiung Medical University, Kaohsiung, 807, Taiwan

\section{Acknowledgements}

Lu-Chuan Ceng was partially supported by the Innovation Program of Shanghai Municipal Education Commission (15ZZ068), Ph.D. Program Foundation of Ministry of Education of China (20123127110002), and Program for Outstanding Academic Leaders in Shanghai City (15XD1503100). Ching-Feng Wen was partially supported by the grant MOST 103-2115-M-037-001. This study is supported partially by Kaohsiung Medical University 'Aim for the Top Universities Grant, grant No. KMU-TP103F01':

Received: 13 January 2015 Accepted: 30 March 2015 Published online: 22 April 2015

\section{References}

1. Lions, JL: Quelques Méthodes de Résolution des Problèmes aux Limites Non Linéaires. Dunod, Paris (1969)

2. Glowinski, R: Numerical Methods for Nonlinear Variational Problems. Springer, New York (1984)

3. Takahashi, W: Nonlinear Functional Analysis. Yokohama Publishers, Yokohama (2000)

4. Korpelevich, GM: The extragradient method for finding saddle points and other problems. Matecon 12, 747-756 (1976)

5. Ceng, LC, Hadjisavvas, N, Wong, NC: Strong convergence theorem by a hybrid extragradient-like approximation method for variational inequalities and fixed point problems. J. Glob. Optim. 46, 635-646 (2010)

6. Ceng, LC, Ansari, QH, Schaible, S: Hybrid extragradient-like methods for generalized mixed equilibrium problems, system of generalized equilibrium problems and optimization problems. J. Glob. Optim. 53, 69-96 (2012)

7. Ceng, LC, Petruşel, A: Relaxed extragradient-like method for general system of generalized mixed equilibria and fixed point problem. Taiwan. J. Math. 16(2), 445-478 (2012)

8. Ceng, LC, Wang, CY, Yao, JC: Strong convergence theorems by a relaxed extragradient method for a general system of variational inequalities. Math. Methods Oper. Res. 67(3), 375-390 (2008)

9. Nadezhkina, N, Takahashi, W: Weak convergence theorem by an extragradient method for nonexpansive mappings and monotone mappings. J. Optim. Theory Appl. 128, 191-201 (2006)

10. Zeng, LC, Yao, JC: Strong convergence theorem by an extragradient method for fixed point problems and variational inequality problems. Taiwan. J. Math. 10(5), 1293-1303 (2006)

11. Ceng, LC, Al-Homidan, S: Algorithms of common solutions for generalized mixed equilibria, variational inclusions, and constrained convex minimization. Abstr. Appl. Anal. 2014, Article ID 132053 (2014)

12. Ceng, LC, Yao, JC: A relaxed extragradient-like method for a generalized mixed equilibrium problem, a general system of generalized equilibria and a fixed point problem. Nonlinear Anal. 72, 1922-1937 (2010)

13. Ceng, LC, Kong, ZR, Wen, CF: On general systems of variational inequalities. Comput. Math. Appl. 66, 1514-1532 (2013)

14. Yao, Y, Liou, YC, Kang, SM: Approach to common elements of variational inequality problems and fixed point problems via a relaxed extragradient method. Comput. Math. Appl. 59, 3472-3480 (2010)

15. Ceng, LC, Ansari, QH, Wong, MM, Yao, JC: Mann type hybrid extragradient method for variational inequalities, variational inclusions and fixed point problems. Fixed Point Theory 13(2), 403-422 (2012)

16. Ceng, LC, Ansari, QH, Yao, JC: An extragradient method for solving split feasibility and fixed point problems. Comput. Math. Appl. 64(4), 633-642 (2012)

17. Ceng, LC, Ansari, QH, Yao, JC: Relaxed extragradient methods for finding minimum-norm solutions of the split feasibility problem. Nonlinear Anal. 75(4), 2116-2125 (2012)

18. Ceng, LC, Ansari, QH, Yao, JC: Relaxed extragradient iterative methods for variational inequalities. Appl. Math. Comput. 218, 1112-1123 (2011)

19. Ceng, LC, Ansari, QH, Wong, NC, Yao, JC: An extragradient-like approximation method for variational inequalities and fixed point problems. Fixed Point Theory Appl. 2011, Article ID 22 (2011)

20. Cai, G, Bu, SQ: Strong and weak convergence theorems for general mixed equilibrium problems and variational inequality problems and fixed point problems in Hilbert spaces. J. Comput. Appl. Math. 247, 34-52 (2013)

21. Blum, E, Oettli, W: From optimization and variational inequalities to equilibrium problems. Math. Stud. 63(1-4), 123-145 (1994)

22. Oettli, W: A remark on vector-valued equilibria and generalized monotonicity. Acta Math. Vietnam. 22, $215-221$ (1997) 
23. Moudafi, A: Weak convergence theorems for nonexpansive mappings and equilibrium problems. J. Nonlinear Convex Anal. 9(1), 37-43 (2008)

24. Takahashi, S, Takahashi, W: Strong convergence theorem for a generalized equilibrium problem and a nonexpansive mapping in a Hilbert space. Nonlinear Anal. 69, 1025-1033 (2008)

25. Ceng, LC, Yao, JC: A hybrid iterative scheme for mixed equilibrium problems and fixed point problems. J. Comput. Appl. Math. 214, 186-201 (2008)

26. Moudafi, A, Mainge, P-E: Strong convergence of an iterative method for hierarchical fixed point problems. Pac. J. Optim. 3(3), 529-538 (2007)

27. Ceng, LC, Petruşel, A, Yao, JC: Iterative approaches to solving equilibrium problems and fixed point problems of infinitely many nonexpansive mappings. J. Optim. Theory Appl. 143, 37-58 (2009)

28. Moudafi, A, Mainge, P-E: Towards viscosity approximations of hierarchical fixed point problems. Fixed Point Theory Appl. 2006, Article ID 95453 (2006)

29. Atsushiba, S, Takahashi, W: Strong convergence theorems for a finite family of nonexpansive mappings and applications. B.N. Prasad Birth Centenary Commemoration Volume. Indian J. Math. 41(3), 435-453 (1999)

30. Rattanaseeha, K: The general iterative methods for equilibrium problems and fixed point problems of countable family of nonexpansive mappings in Hilbert spaces. J. Inequal. Appl. 2013, Article ID 153 (2013)

31. Cianciaruso, F, Marino, G, Muglia, L, Yao, Y: A hybrid projection algorithm for finding solutions of mixed equilibrium problem and variational inequality problem. Fixed Point Theory Appl. 2010, Article ID 383740 (2010)

32. Verma, RU: On a new system of nonlinear variational inequalities and associated iterative algorithms. Math. Sci. Res. Hot-Line 3(8), 65-68 (1999)

33. Marino, G, Muglia, L, Yao, Y: Viscosity methods for common solutions of equilibrium and variational inequality problems via multi-step iterative algorithms and common fixed points. Nonlinear Anal. 75, 1787-1798 (2012)

34. Yao, Y, Liou, Y-C, Marino, G: Two-step iterative algorithms for hierarchical fixed point problems and variational inequality problems. J. Appl. Math. Comput. 31(1-2), 433-445 (2009)

35. Rockafellar, RT: Monotone operators and the proximal point algorithms. SIAM J. Control Optim. 14, 877-898 (1976)

36. Huang, NJ: A new completely general class of variational inclusions with noncompact valued mappings. Comput. Math. Appl. 35(10), 9-14 (1998)

37. Zeng, LC, Guu, SM, Yao, JC: Characterization of $\mathrm{H}$-monotone operators with applications to variational inclusions. Comput. Math. Appl. 50(3-4), 329-337 (2005)

38. Ceng, LC, Guu, SM, Yao, JC: Iterative algorithm for finding approximate solutions of mixed quasi-variational-like inclusions. Comput. Math. Appl. 56, 942-952 (2008)

39. Ceng, LC, Guu, SM, Yao, JC: Iterative approximation of solutions for a class of completely generalized set-valued quasi-variational inclusions. Comput. Math. Appl. 56, 978-987 (2008)

40. Zhang, SS, Lee, JHW, Chan, CK: Algorithms of common solutions for quasi variational inclusions and fixed point problems. Appl. Math. Mech. 29, 571-581 (2008)

41. Ceng, LC, Lin, YC, Wen, CF: Iterative methods for triple hierarchical variational inequalities with mixed equilibrium problems, variational inclusions, and variational inequalities constraints. J. Inequal. Appl. 2015, Article ID 16 (2015)

42. Moudafi, A: Viscosity approximation methods for fixed-points problems. J. Math. Anal. Appl. 241(1), 46-55 (2000)

43. Xu, HK: Viscosity approximation methods for nonexpansive mappings. J. Math. Anal. Appl. 298, 279-291 (2004)

44. Colao, V, Marino, G, Xu, HK: An iterative method for finding common solutions of equilibrium and fixed point problems. J. Math. Anal. Appl. 344, 340-352 (2008)

45. Takahashi, W, Toyoda, M: Weak convergence theorems for nonexpansive mappings and monotone mappings. J. Optim. Theory Appl. 118(2), 417-428 (2003)

46. Ceng, LC, Guu, SM, Yao, JC: Finding common solutions of a variational inequality, a general system of variational inequalities, and a fixed-point problem via a hybrid extragradient method. Fixed Point Theory Appl. 2011, Article ID $626159(2011)$

47. Ceng, LC, Petruşel, A, Yao, JC: Composite viscosity approximation methods for equilibrium problem, variational inequality and common fixed points. J. Nonlinear Convex Anal. 15, 219-240 (2014)

48. Al-Mazrooei, AE, Bin Dehaish, BA, Latif, A, Yao, JC: Hybrid viscosity approximation methods for general systems of variational inequalities in Banach spaces. Fixed Point Theory Appl. 2013, Article ID 258 (2013)

49. Ceng, LC, Yao, JC: On the triple hierarchical variational inequalities with constraints of mixed equilibria, variational inclusions and systems of generalized equilibria. Tamkang J. Math. 45(3), 297-334 (2014)

50. Ceng, LC, Latif, A, Ansari, QH, Yao, JC: Hybrid extragradient method for hierarchical variational inequalities. Fixed Point Theory Appl. 2014, Article ID 222 (2014)

51. Ceng, LC, Sahu, DR, Yao, JC: A unified extragradient method for systems of hierarchical variational inequalities in a Hilbert space. J. Inequal. Appl. 2014, Article ID 460 (2014)

52. Marino, G, Xu, HK: Weak and strong convergence theorems for strict pseudo-contractions in Hilbert spaces. J. Math. Anal. Appl. 329, 336-346 (2007)

53. Goebel, K, Kirk, WA: Topics on Metric Fixed-Point Theory. Cambridge University Press, Cambridge (1990)

54. Marino, G, Xu, HK: A general iterative method for nonexpansive mappings in Hilbert spaces. J. Math. Anal. Appl. 318(1), 43-52 (2006)

55. Xu, HK: Iterative algorithms for nonlinear operators. J. Lond. Math. Soc. 66(2), 240-256 (2002)

56. Barbu, V: Nonlinear Semigroups and Differential Equations in Banach Spaces. Noordhoff, Groningen (1976) 